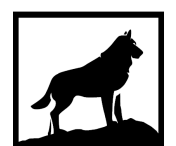

Michigan

Technological

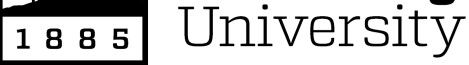

Michigan Technological University

Digital Commons @ Michigan Tech

\title{
LABORATORY, COMPUTATIONAL AND THEORETICAL INVESTIGATIONS OF ICE NUCLEATION AND ITS IMPLICATIONS FOR MIXED PHASE CLOUDS
}

Fan Yang

Michigan Technological University, fyang6@mtu.edu

Copyright 2017 Fan Yang

Recommended Citation

Yang, Fan, "LABORATORY, COMPUTATIONAL AND THEORETICAL INVESTIGATIONS OF ICE NUCLEATION AND ITS IMPLICATIONS FOR MIXED PHASE CLOUDS", Open Access Dissertation, Michigan Technological University, 2017.

https://doi.org/10.37099/mtu.dc.etdr/477

Follow this and additional works at: https://digitalcommons.mtu.edu/etdr

Part of the Atmospheric Sciences Commons 


\title{
LABORATORY, COMPUTATIONAL AND THEORETICAL INVESTIGATIONS OF ICE NUCLEATION AND ITS IMPLICATIONS FOR MIXED PHASE CLOUDS
}

By

Fan Yang

\begin{abstract}
A DISSERTATION
Submitted in partial fulfillment of the requirements for the degree of DOCTOR OF PHILOSOPHY

In Atmospheric Sciences

MICHIGAN TECHNOLOGICAL UNIVERSITY
\end{abstract}

2017

(C) 2017 Fan Yang 

This dissertation has been approved in partial fulfillment of the requirements for the Degree of DOCTOR OF PHILOSOPHY in Atmospheric Sciences.

Department of Physics

Dissertation Advisor: Dr. Raymond A. Shaw

Committee Member: Dr. Alex B. Kostinski

Committee Member: Dr. Will H. Cantrell

Committee Member: Dr. Mikhail Ovchinnikov

Department Chair: Dr. Ravindra Pandey 



\section{Dedication}

To my dearest Pei Hou

For your support, encouragement, and love. 



\section{Contents}

List of Figures $\ldots \ldots \ldots \ldots \ldots \ldots \ldots \ldots \ldots \ldots \ldots \ldots$

Acknowledgments ............................

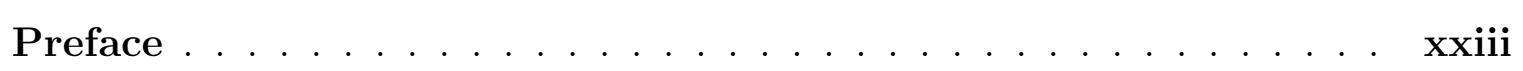

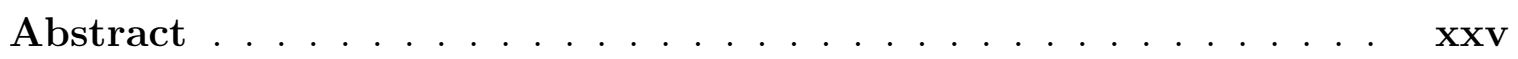

1 Background $\ldots \ldots \ldots \ldots \ldots \ldots$

1.1 Homogeneous Ice Nucleation . . . . . . . . . . . . . . . . . . 3

1.2 Heterogeneous Ice Nucleation $\ldots \ldots \ldots$. . . . . . . . . . 6

1.3 Immersion Nucleation . . . . . . . . . . . . . . . . . . . . . G

1.4 Contact Nucleation . . . . . . . . . . . . . . . . 9

1.5 Pressure Induced Chemical Potential Change . . . . . . . . . . . . 11

1.6 Wetting Phenomenon . . . . . . . . . . . . . . . . . . . . 12

1.7 Electrowetting And Electrofreezing . . . . . . . . . . . . 14

1.8 Droplet Vibration On A Vertically-Oscillating Plane . . . . . . . . 17 
2 Ice Nucleation At The Contact Line Triggered By Transient Electrowetting Fields ..................... 19

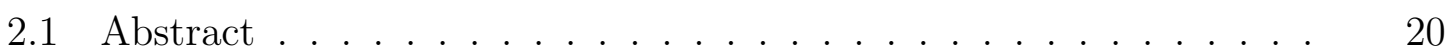

2.2 Introduction . . . . . . . . . . . . . . . . . 20

2.3 Experimental Methods . . . . . . . . . . . . . . . 22

2.4 Results and Discussion . . . . . . . . . . . . . . . . 24

2.5 Acknowledgement ...................... 36

3 Non-thermal Ice Nucleation Observed At Distorted Contact Lines Of Supercooled Water Drops . . . . . . . . . . . . . 37

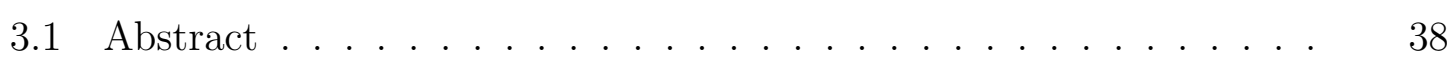

3.2 Introduction . . . . . . . . . . . . . . . . . . 39

3.3 Experimental Methods ... . . . . . . . . . . . . . 40

3.4 Results and Discussion . . . . . . . . . . . . . . . 42

3.5 Acknowledgement ........................... 59

4 Minimalist Model of Ice Microphysics in Mixed-Phase Stratiform Clouds ............................ 61

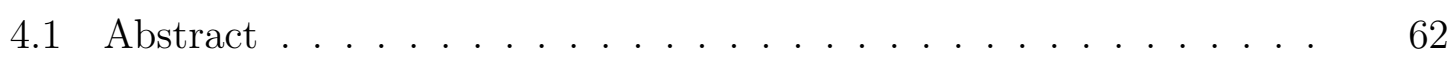

4.2 Introduction . . . . . . . . . . . . . . . . 62

4.3 Model Description ..................... 64

4.4 Comparison Of Results With LES Cloud Model And ISDAC Observations 


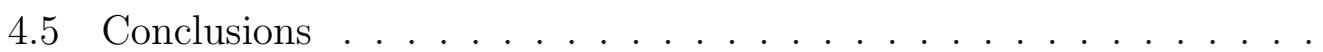

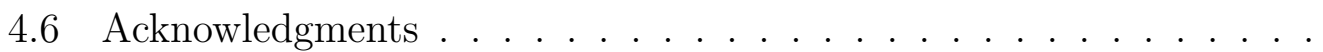

5 Microphysical Consequences Of The Spatial Distribution Of Ice Nucleation In Mixed-Phase Stratiform Clouds

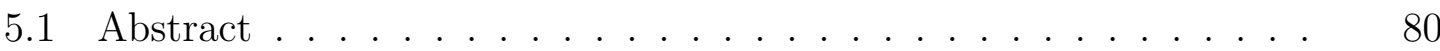

5.2 Introduction . . . . . . . . . . . . . . . . . 80

5.3 Analytical Model Of Ice Growth In A Mixed-Phase Stratiform Cloud

5.4 Lagrangian Ice Particle Tracking In LES Time Dependent Field . .

5.5 Influence Of The Spatial Distribution Of Ice Nucleation . . . . . . .

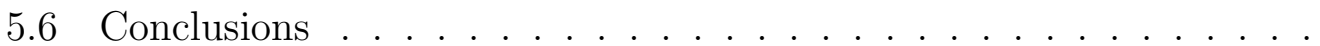

5.7 Acknowledgements ...................... 101

\section{Long-Lifetime Ice Particles In Mixed-Phase Stratiform Clouds:} Quasi-Steady And Recycled Growth

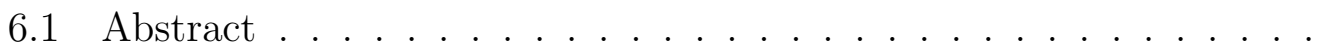

6.2 Introduction . . . . . . . . . . . . . . . .

6.3 Model Setup For Langrangian Ice Particle Tracking In 3D Time Dependent Field . . . . . . . . . . . . . . . . . . .

6.4 Long Lifetime Ice Particles In A 3D Time Dependent Velocity Field

6.5 Long Lifetime Ice Particles In A 2D Idealized Velocity Field . . . .

6.6 Analytical Solutions For Trajectories Of Constant-Size Ice Particles In Simple Velocity Fields 
6.6.1 Solid Body Rotation Field . . . . . . . . . . . 138

6.6 .2 Irrotational Field . . . . . . . . . . . . . . . . 142

6.7 Ice Water Content In A Coupled Versus Decoupled Boundary Layer 146

6.8 Conclusions ............................. 153

6.9 Acknowledgments . . . . . . . . . . . . . . . 158

7 Discussion . . . . . . . . . . . . . . . . . . . . . 159

7.1 Conclusion . . . . . . . . . . . . . . . . . . . 159

7.2 Future Research . . . . . . . . . . . . . . . 162

7.3 Additional Research Topics . . . . . . . . . . . . . 166

References .............................. 171 


\section{List of Figures}

1.1 Gibbs free energy $\Delta G_{i}$ for homogeneous freezing at different tempera-

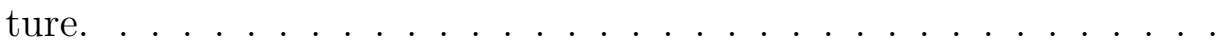

1.2 Homogeneous ice nucleation rate with and without $g_{a c t} \ldots \ldots \ldots$

1.3 Sketch of two traditional electrofreezing setups: A is parallel plane electric field and B is high voltage wire inside of cloud chamber. . .

1.4 A) shows the water droplet on a dielectric substrate without an electric field. B) shows the standard setup for electrowetting, with a highvoltage electrode in the droplet and a grounded plane beneath the

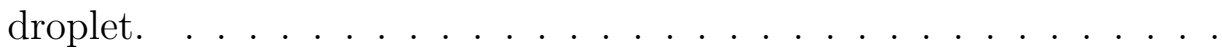

2.1 a) Sketch of the experimental setup from the side, illustrating the electrowetting geometry. b) Top view of a crystalizing droplet from the high speed camera. . . . . . . . . . . . .

2.2 Natural freezing temperature for different voltages (blue points) and freezing fraction when turning on/off the electric field at a constant temperature. . . . . . . . . . . . . . . 
2.3 Time-lapse views of crystallization after switching on three voltages $(600 \mathrm{~V}, 800 \mathrm{~V}$ and $1000 \mathrm{~V})$ at $-10{ }^{\circ} \mathrm{C}$ (left) and $-15{ }^{\circ} \mathrm{C}$ (right). The images are taken with a $5 \mathrm{kHz}$ high speed camera. Each frame in one column is separated by $10 \mathrm{~ms} . \ldots \ldots \ldots \ldots \ldots$

3.1 Response of a $30 \mu L \mathrm{~A}$ ) pure water and B) water with $10 \mathrm{mg} / \mathrm{ml}$ pump oil on a silica glass substrate for different amplitudes at $30 \mathrm{~Hz}$ and $-17.0 \pm 0.5{ }^{\circ} \mathrm{C} \ldots \ldots \ldots \ldots \ldots \ldots \ldots \ldots \ldots \ldots \ldots \ldots$

3.2 Side view of the response of a $30 \mu L$ drop changes with amplitude at three different amplitudes. . . . . . . . . . . . . . . .

3.3 Individual video frames showing water (first column) and water with 10 $\mathrm{mg} / \mathrm{mL}$ pump oil (second column) at different stages of oscillation on silica glass substrate with $30 \mathrm{~Hz}$ and $v_{\max }=56.0 \mathrm{~cm} / \mathrm{s}$ at $-17.0 \pm 0.5$

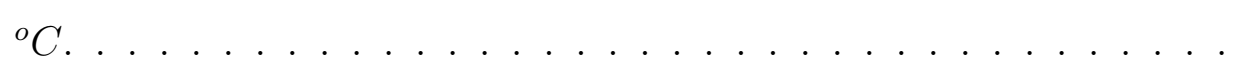

3.4 Response of a $30 \mu L$ water with $10 \mathrm{mg} / \mathrm{ml}$ mineral oil on a silica glass substrate for different amplitudes at $30 \mathrm{~Hz}$ and $-17.0 \pm 0.5{ }^{\circ} \mathrm{C}$. . .

3.5 Individual video frames showing water with $10 \mathrm{mg} / \mathrm{mL}$ mineral oil at different stages of oscillation on silica glass substrate with $30 \mathrm{~Hz}$ and $v_{\max }=56.0 \mathrm{~cm} / \mathrm{s}$ at $-17.0 \pm 0.5^{\circ} \mathrm{C} \ldots \ldots \ldots$ 
3.6 Effect of oil concentration on freezing fraction (blue lines) and freezing delay time after start of oscillation (red lines) with vibration of $30 \mathrm{~Hz}$ and $v_{\max }=56.0 \mathrm{~cm} / \mathrm{s}$ at $-17.0 \pm 0.5^{\circ} \mathrm{C}$. Each case is repeated ten times. . . . . . . . . . . . . . . . .

3.7 Example of macroscopic pinning behavior during oscillation of A) the water-oil mixture on a silica glass substrate and B) pure water on a PDMS substrate. C) Sketch of a curved contact line with indication of local pressure perturbations (+ or -$)$. The scale is arbitrary, but can be expected to extend to nanometers, where the equivalent temperature change is of order degrees K. . . . . . . . . . . . . .

3.8 A) Response of a $30 \mu L$ pure water droplet on a PDMS substrate for different amplitudes at $30 \mathrm{~Hz}$. Format and line styles are as in Figure 3.1. B) Fraction of droplets that freeze for different amplitudes with $30 \mathrm{~Hz}$ at three temperatures. . . . . . . . . . . . . .

4.1 (a)Height of ice particle above cloud base vs time. Updraft velocity decreases linearly from $v_{0}=0.3 \mathrm{~m} / \mathrm{s}$ at the base to zero at the top. Blue line represents ice formed at cloud top, red at cloud base. Black line is based on quasi-steady state (Equation 4.2). (b) Diameter at cloud base of ice particles which forms at cloud top (blue) and cloud base (red) under different background updraft velocity $v_{0} \ldots \ldots$. . 
4.2 Ice water content and ice number concentration relationship from LES. (a) and (c) are accumulation zone region. (b) and (d) are selective accumulation zone region. Black lines in (c) and (d) are best fitted 2.5 slope lines. Colors in (a) and (b) represent updraft velocity, while colors in (c) and (d) mean altitude. The cloud base and top are at about $600 \mathrm{~m}$ and $800 \mathrm{~m}$, respectively. . . . . . . . . .

4.3 $w_{i}$ and $n_{i}$ relationship from Flight 31 ISDAC. Solid and dashed black lines represent 2.5 slope and 1.0 slope respectively. . . . . . . . . .

$4.4 w_{i}$ and $n_{i}$ relationship for two ice nucleation rates. Blue points are from LES with $\phi / \tau=2 * 10^{-9}$ and red points with $\phi / \tau=10^{-8}$. Solid and dashed lines are best fitted 2.5 slope lines. . . . . . . . . . . .

5.1 Mean vertical profiles of a) Temperature, b) Pressure, c) liquid cloud fraction and d) relative humidity respective to liquid water and ice.

$5.2 w_{i}$ and $n_{i}$ relationship at three different layers from the Lagrangian particle tracking method in LES time dependent field. Black line is based on Equation 5.1 and red line is from Equation 5.10. Green dot corresponds to the average $\log \left(n_{i}\right)$ and $\log \left(w_{i}\right)$ at each level. . . . 
5.3 Relationship between $w_{i}$ ( $k g$ per grid) and $n_{i}$ (\# per grid) at 650 $m$ (g,h,i), $700 m$ (d,e,f) and $750 m$ (a,b,c) for three different seeding places.a), d) and g) are uniform seeding in the cloud layer; b), e) and h) are seeding at cloud top; c), f) and i) are seeding at cloud base. Red line is calculated from Equation 5.10, and brown dash line is 1.0 slope line, while grey line is -5 slope for comparison. . . . . . . . .

5.4 Relationship between $w_{i}$ ( $k g$ per grid) and $n_{i}$ (\# per grid) at $700 \mathrm{~m}$ for different threshold radii from the Lagrangian particle tracking model. Red line is calculated from Equation 5.10 and the brown dash line is 1.0 slope for comparison. Circle, star, triangle and square represent the average of $\log \left(w_{i}\right)$ and $\log \left(n_{i}\right)$ for blue, pink, green and red dots separately. . . . . . . . . . . . . . . . .

6.1 (a) Fraction of ice particles remaining in the cloud. The black line is from the LES time dependent field. (b) PDF of ice particle lifetimes. Different colors represent different recycling numbers. (c) Ice particle survival fraction after a specific lifetime $\tau$, defined as $\chi(\tau)=1-$ $\int_{0}^{\tau} P D F(t) d t \ldots \ldots \ldots \ldots \ldots \ldots$ 
6.2 Time evolution of the probability density function for ice particle radius in the mixed phase cloud. The black curve is the growth of an ice particle under a constant supersaturation, which is set to be the initial supersaturation in the mixed phase cloud. The block dots indicate the maximum value of the PDF at each time. . . . . . . . . . .

6.3 Radius versus time for ten long-lifetime ice particles in the mixed phase cloud. The ice particle radius is plotted only for the times when it is in the mixed phase cloud, with dashed lines joining the regions between which the ice particles are below liquid cloud base. Color here represents the recycling number. The large star symbols represent the average ice particle radius over the whole mixed phase cloud region, and its color represents the average recycling number at that time. The grey shading indicate the full range of ice particle size distribution at each time as shown in Figure 6.2. . . . . . . . . . . . . . . . . .

6.4 Initial horizontal seeding location for long lifetime ice particles. Background color represents the initial air vertical velocity. . . . . . . .

6.5 Probability density function of vertical air velocity conditioned on ice particle location, for ice particles with different ranges of lifetime. The gray dashed line is the initial vertical air velocity for all ice particles seeded in the cloud, which is centered on $w=0 . \ldots . . . .$. 
6.6 Velocity field in the idealized 2D field with $v_{\max }=2 \mathrm{~ms}^{-1}$. The arrows indicate the wind direction and magnitude. The black dashed line (at $420 \mathrm{~m}$ ) represents the level above which the environment is supersaturated with respect to ice, below is the region of subsaturation with respect to ice, and the shaded area denotes the mixed-phase region.

$6.7 \chi$ versus lifetime in $2 \mathrm{D}$ field with different maximum velocity $v_{\max }$. Here the thick blue line (all) includes both recycling and non-recycling ice particles, and the thin red line (0) only includes non-recycling ice

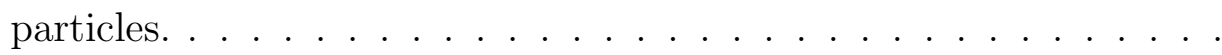

6.8 Locations of recycled particles when first seeded for three different $v_{\max }$ : a) $1.0 \mathrm{~ms}^{-1}$, b) $2.0 \mathrm{~ms}^{-1}$, c) $4.0 \mathrm{~ms}^{-1}$. Color represents the recycling number. It should be mentioned that the z-axis is restricted to the mixed-phase layer and the x-axis is limited to the upward branch of the circulation in Figure 6.6. . . . . . . . . . . . . .

6.9 Trajectories of three different ice particles in an analytical velocity field with $v_{\max }=2.0 \mathrm{~ms}^{-1}$ (see Equation 6.2 for explanation). Red dots represent initial seeding locations (x,z) at (a) (800 m, $790 \mathrm{~m}),(\mathrm{b})(1200$ $\mathrm{m}, 720 \mathrm{~m})$, and $(\mathrm{c})(1100 \mathrm{~m}, 650 \mathrm{~m})$. The color bar here represents the radius of the ice particle. Subcloud region is below $600 \mathrm{~m}$ and subsaturated region with respect to ice is below $420 \mathrm{~m}$, illustrated in Figure 6.6, . . . . . . . . . . . . . . . . . . . 
6.10 Height versus radius for two types of long lifetime ice particles with $v_{\max }=2.0 \mathrm{~ms}^{-1}$. Colors here represent the air vertical velocity. Type 1 is initially seeding at $(800 \mathrm{~m}, 790 \mathrm{~m})$ and Type 2 is seeded at (1200 $\mathrm{m}, 720 \mathrm{~m}$ ). Subcloud region (below $600 \mathrm{~m}$ ) and subsaturated region with respect to ice (below $420 \mathrm{~m}$ ) are the same as in Figure 6.6. . .

6.11 Height versus radius for four specific ice particles in the 3D time dependent field. Grey shading indicate the mixed phase cloud region and the colorbar represents the vertical velocity (in $m s^{-1}$ ). Red dots represent the initial seeding locations. . . . . . . . . . . . . . .

6.12 (a) Available range of $r$ for a closed trajectory in the solid body rotation field $v_{a, \theta}=\omega r$, where $\omega=0.01 s^{-1}$. Black line is $g(r)=0$. Details of $g_{1}, g_{2}, r_{\min }$ and $r_{\max }$ are described in the text. (b) Particle trajectory in the field (black dashed line, with blue and green circles denoting the limiting radii of curvature). Initial particle with constant terminal velocity $v_{t 0}=0.1 \mathrm{~ms}^{-1}$ is located at $x=50 \mathrm{~m}, z=50 \mathrm{~m}$. Green and blue lines are bounded circles with radii of $r_{\min }$ and $r_{\max }$. Gray dots are simulated trajectory, and black dots are from the analytical solution. Red point represents the initial location. (c) Trajectories of particles with three different terminal velocities $v_{t 0}=0.10 .3,0.5$ $m s^{-1} \ldots \ldots \ldots \ldots \ldots \ldots \ldots \ldots$ 
6.13 (a) Available range of $r$ for a closed trajectory in simple irrotational field $v_{a, \theta}=B_{0} / r$, where $B_{0}=100 m^{2} s^{-1}$. Black line is $g(r)=\ln r$. Details of $g_{1}, g_{2}, r_{\min }$ and $r_{\max }$ are described in the text. (b) Particle trajectory in the field (black dashed line, with blue and green circles denoting the limiting radii of curvature). Initial particle with constant terminal velocity $v_{t 0}=0.1 \mathrm{~ms}^{-1}$ is located at $x=50 \mathrm{~m}, z=50$ m. Symbols are the same as those in Figure 6.12, (c) Trajectories of particles with three different terminal velocities $v_{t 0}=0.10 .3,0.5$ $m s^{-1} \ldots \ldots \ldots \ldots \ldots \ldots \ldots \ldots$

6.14 Velocity field when the eddy is decoupled from the surface. The eddy is developed between $400 \mathrm{~m}$ and $800 \mathrm{~m}$, and below $400 \mathrm{~m}$ the air is still. The black dashed line represents where the environment is saturated with respect to ice, and the shaded area denotes the mixedphase region.

6.15 a) Time variation of total ice water mass ratio in the mixed phase cloud (between $600 \mathrm{~m}$ and $800 \mathrm{~m}$ ) for different cases with respect to the base case: AR2, coupled, $v_{\max }=2.0 \mathrm{~m} / \mathrm{s}$. Case details are described in the text. b) The mass fraction of recycling ice particles in the mixed phase cloud for different cases. . . . . . . . . . . . . . . . 
$6.16 \chi$ versus lifetime for all ice particles (solid line) and non-recycle ice particles (dashed line). Different colors represent different cases: magenta is for the 'AR2, coupled, $2.0 \mathrm{~ms}^{-1}$ ' case, green line is for 'AR4, decoupled, $2.0 \mathrm{~ms}^{-1}$ ' case, and black is for 'AR4, decoupled, $0.5 \mathrm{~ms}^{-1}$ ',

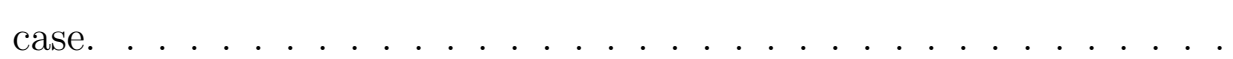

7.1 Ice formation in front of the main water-ice interface. The water droplet is freezing at $-14{ }^{\circ} \mathrm{C}$ on a silicon substrate. . . . . . . . 


\section{Acknowledgments}

I would like to thank my advisor Dr. Raymond Shaw. I'm very lucky that I have the chance to work with him. He explored my scope of knowledge in atmospheric science, inspired my interest in cloud physics, encouraged me to do research in many directions, and guided me to the right path when I met some problems. He's a mentor in my research and a friend in my life.

I would like to thank all my committee members Dr. Alex Kostinski, Dr. Will Cantrell and Dr. Mikhail Ovchinnikov for my research discussion. Thanks to Dr. Alex Kostinski who deepened my understanding of physics and mathematics, and also thanks for extremely useful discussion about ice nucleation theoretically. Thanks to Dr. Will Cantrell who gave me suggestion and help in ice nucleation experiment, and also provided me the chance to learn lots of ice-nucleation-related experimental work in his group. Thanks to Dr. Mikhail Ovchinnikov who guided me how to modeling clouds using SAM, and helped me with the mixed-phase-cloud simulation and discussion.

My sincere thanks goes to all members in cloud physics group for their assistance and helpful discussion, including Dr. Colin Gurganus, Dr. Kelken Chang, Dr. Dennis Niedermeier, Dr. Jiang Lu, Dr. Matt Beals, Mr. Nick Black, Mr. David Ciochetto, 
Mr. Neel Desai, Mr. Kamal Kant Chandrakar, Ms. Susanne Glienke, Mr. Subin Thomas, Mr. Owen Cruikshank, Mr. Adam Laxo, Mr. Michael Adler, Mr. Joe Charnawskas. Special thanks goes to Mr. Sukong Chong, who prepared the graphene covered glass substrate for my experiment, and to Dr. Weilue He (MTU Biomedical Engineering), who helped in designing the glass substrate with glue wall and preparing the magic oil solutions, and to Dr. Joseph Niehaus for helpful discussion about ice nucleation experiment.

I should also thank Dr. Ravindra Pandey, Dr. Yoke Khin Yap, Dr. Andrew Barnard (MTU Mechanical EngineeringEngineering Mechanics), Dr. Jaroslaw W. Drelich (MTU Materials Science and Engineering), Ms Andrea Lappi, Mr. Jesse Nordeng, Mr. Slough, and Mr. Scott Rutterbush, who facilitate my research in one way or another. Thanks to the MTU Atmospheric Science Program, the MTU Physics Department, the MTU Graduate School, the Department of Energy.

Last but not the least, I would like to thank my family for supporting me spiritually. Finally and most importantly, I would like to thank my wife Pei Hou, for her support and encouragement in my research, and for her tolerance, understanding and love in my life. 


\section{Preface}

In this dissertation I will present the work I've done in the Cloud Physics Lab at MTU. Chapter 1 briefly describes the background for my research. Chapter 2 is based on a paper published in Applied Physics Letters (Yang et al., 2015b). Chapter 4 is based on a paper published in Geophysical Research Letters (Yang et al., 2013). Chapter 5 is based a paper published in Geophysical Research Letters (Yang et al., 2014). Chapter 6 is based on a paper published in Journal of Geophysical Research Atmospheres (Yang et al., 2015a). Part of Chapter 7 is based on a paper published in Atmospheric Chemistry and Physics (Yang et al., 2016). Data collecting, data analyzing and paper writing were done by me with the help of Dr. Raymond Shaw and other co-authors. Chapter 3 is based on a manuscript that we just submitted to a journal and is currently under review. We sincerely thank our co-authors for their contributions. 



\section{Abstract}

Ice particles in atmospheric clouds play an important role in determining cloud lifetime, precipitation and radiation. It is therefore important to understand the whole life cycle of ice particles in the atmosphere, e.g., where they come from (nucleation), how they evolve (growth), and where they go (precipitation). Ice nucleation is the crucial step for ice formation, and in this study, we will mainly focus on ice nucleation in the lab and its effect on mixed-phase stratiform clouds.

In the first half of this study, we investigate the relevance of moving contact lines (i.e., the region where three or more phases meet) on the phenomenon of contact nucleation. High speed video is used to investigate heterogeneous ice nucleation in supercooled droplets resting on cold substrates under two different dynamic conditions: droplet electrowetting and droplet vibration. The results show that contact-line motion is not a sufficient condition to trigger ice nucleation, while locally curved contact lines that can result from contact-line motion are strongly related to ice nucleation. We propose that pressure perturbations due to locally curved contact lines can strongly enhance the ice nucleation rate, which gives another interpretation for the mechanism for contact nucleation. Corresponding theoretical results provide a quantitative connection between pressure perturbations and temperature, providing a useful tool for ice nucleation calculations in atmospheric models. 
In this second half of the study, we build a minimalist model for long lifetime mixedphase stratiform clouds based on stochastic ice nucleation. Our result shows that there is a non-linear relationship between ice water contact and ice number concentration in the mixed-phase cloud, as long as the volume ice nucleation rate is constant. This statistical property may help identify the source of ice nuclei in mixed-phase clouds. In addition, results from Lagrangian ice particle tracking in time dependent fields show that long lifetime ice particles exist in mixed-phase stratiform clouds. We find that small ice particle can be trapped in eddy-like structures. Whether ice particles grow or sublimate depends on the thermodynamic field in the trapping region. This dynamic-thermodynamic coupling effect on the lifetime of ice particles might explain the fast phase-partition change observed in the mixed phase cloud. 


\section{Chapter 1}

\section{Background}

Ice crystals in clouds play a crucial role in the hydrological circulation and energy balance on Earth[133]. Many physical and chemical processes in clouds, such as precipitation, radiation and photochemical reactions, are related to the mass and number concentration of ice particles[64]. The origin of ice in the atmosphere is mainly due to homogeneous/heterogeneous ice nucleation. It is known that temperature, supersaturation, water activity and ice nuclei type can affect ice nucleation efficiency[11]. However the nucleation mechanism, especially for heterogeneous ice nucleation, is still quite unknown. Research described in this dissertation is aimed at developing a better understanding of ice nucleation mechanisms through laboratory experiments, and to investigate effects of ice nucleation on the properties of mixed-phase stratiform clouds through numerical simulation and through idealized 
theoretical models.

The purpose of this Chapter is to provide some background materials that will help the reader to understand topics in later Chapters. The basic ice nucleation theory is briefly described in Section 1, 1.1 and 1.2. Two common heterogeneous ice nucleation modes in the atmosphere (immersion and contact) are discussed in Section 1.3 and 1.4. The general phenomenon of wetting is introduced in Section 1.6. Two dynamic spreading phenomena generated by electrowetting and vertically-oscillating substrates used in this study are described in Section 1.7 and 1.8. Further background material on ice nucleation and mixed phase clouds, as well as details of the experimental and theoretical approaches used, are provided in the individual chapters.

\subsection{Ice Nucleation}

The solid form of water is ice. A small amount of water, like a cloud droplet, can exist below the water-ice equilibrium temperature. The supercooled water is thermodynamically unstable, also called metastable. The transition from supercooled water to ice starts from ice nucleation. It is challenging to define ice nucleation and some other related concepts precisely, that recently Vali et al. wrote a paper about ice nucleation terminology [127], which in turn generated a large number of comments from the cloud physics community. 
Ice nucleation is more likely to occur at lower temperatures or within larger volumes. People have considered a lowest temperature at which supercooled water can exist [126]. The lowest temperature that pure water can reach is around $-40^{\circ} \mathrm{C}$, where ice forms spontaneously from supercooled water, which is called homogeneous ice nucleation [64]. However, experiments show that small amounts of liquid water can exist much below that temperature [48]. This might be because of the pressure effect

due to the small radius of curvature [69]. With some foreign particles, ice nucleation might occur at a higher temperature, which is called heterogeneous ice nucleation. The basic theory of ice nucleation will be introduced in Section 1.1 and 1.2.

\section{Homogeneous Ice Nucleation}

The Gibbs free energy change for formation of an ice embryo homogeneously is [64]

$$
\Delta G_{i}=-V_{i} n_{i} \Delta \mu+A_{i} \sigma_{I L}
$$

where $V_{i}$ and $A_{i}$ are the volume and surface of the ice embryo, $n_{i}$ is the molar number density of ice and $\sigma_{I L}$ is the surface tension between water and ice. $\Delta \mu$ in the first term on the right hand side is the thermodynamic driver for the phase change, and 
the second term is the energy barrier for the phase change. The chemical potential change between water and ice is related to the supercooling $\Delta T_{s}=T_{0}-T$,

$$
\Delta \mu=\mu_{L}-\mu_{S}=l_{f} \frac{\Delta T_{s}}{T_{0}}
$$

where $l_{f}$ is the latent heat of fusion per molecule. Figure 1.1 shows an example of the Gibbs free energy change for homogeneous freezing at six different temperatures. It can be seen that there exists a maximum Gibbs free energy change, also called the critical point,

$$
\Delta G_{i}^{*}=\frac{16 \pi \sigma_{I L}^{3} \xi}{3\left(n_{i} l_{f} \frac{\Delta T_{s}}{T_{0}}\right)^{2}},
$$

where $\xi$ is a geometrical factor for a non-spherical embryo. This critical energy $\Delta G_{i}^{*}$ is the energy that an ice embryo needs to overcome the energy barrier and trigger ice nucleation. The corresponding critical size is typically several nanometers, and it increases with increasing of temperature.

The ice nucleation rate quantifies how frequently the energy barrier is overcome (i.e., how often ice nucleation is triggered), per unit volume of water, at a given temperature. For homogeneous ice nucleation,

$$
J_{i}(T)=\frac{k_{B} T}{h} A_{I L} n_{s l} Z n_{L} \exp \left(-\frac{\Delta g_{a c t}}{k_{B} T}-\frac{\Delta G_{i}^{*}}{k_{B} T}\right)
$$

where $A_{I L}$ is the area of the ice-liquid interface, $n_{s l}$ is the number density of molecules 


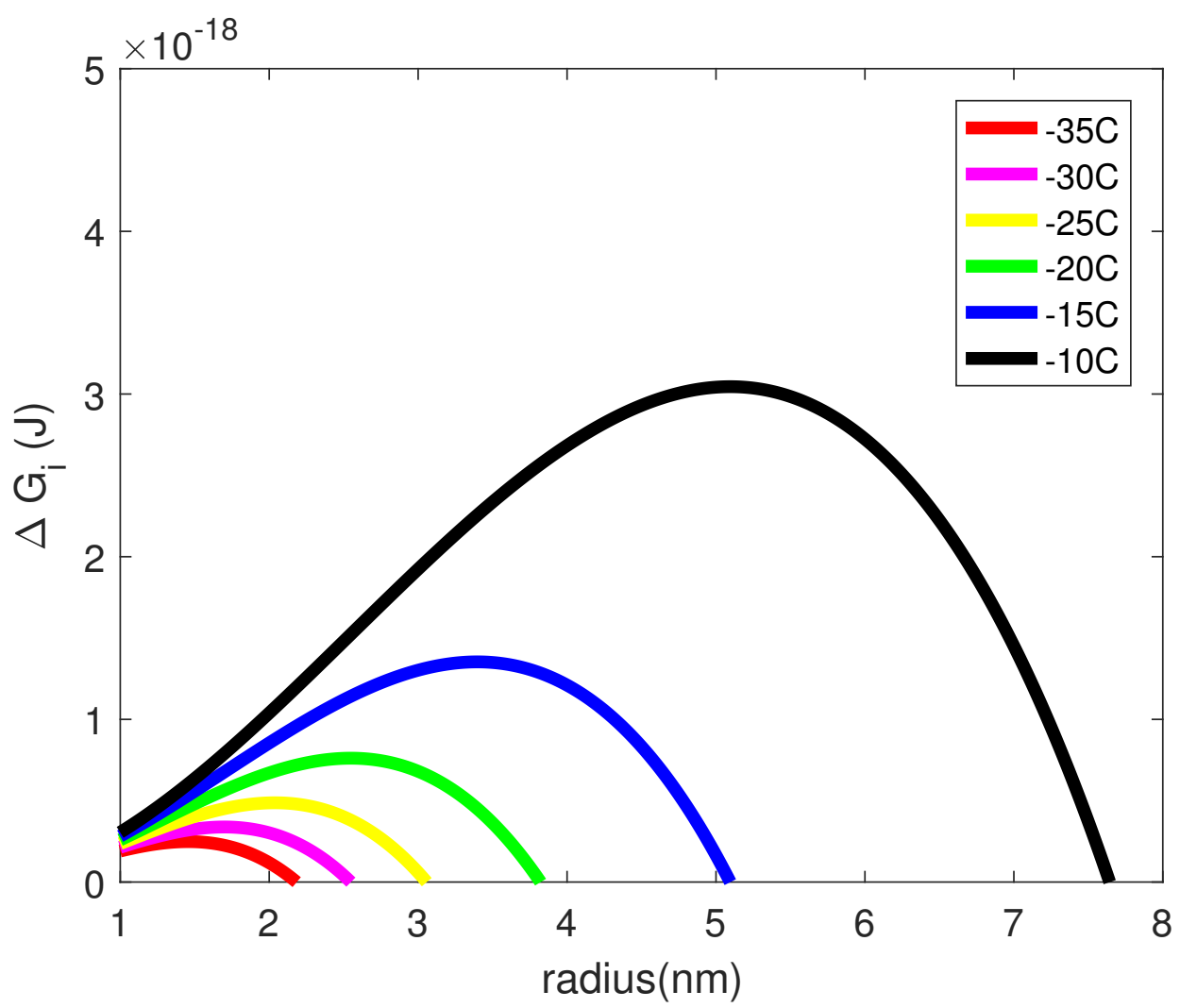

Figure 1.1: Gibbs free energy $\Delta G_{i}$ for homogeneous freezing at different temperature.

in the ice surface, $Z$ is the Zeldovich factor, and $n_{L}$ is the number density of molecules in the liquid phase [64]. The activation energy term $\Delta g_{\text {act }}$ accounts for the rate at which modelucles can be added to the critical ice embryo. Figure 1.2 shows the homogeneous ice nucleation rate calculated based on Li et al. (2013) [71]. It can be seen that $g_{\text {act }}$ plays a significant role in determining $J$, but the true value of $g_{\text {act }}$ is still uncertain [100]. It also indicates that the temperature dependence of $J$ is dominated by the thermodynamic term, and that the activation term mainly causes an offset. 


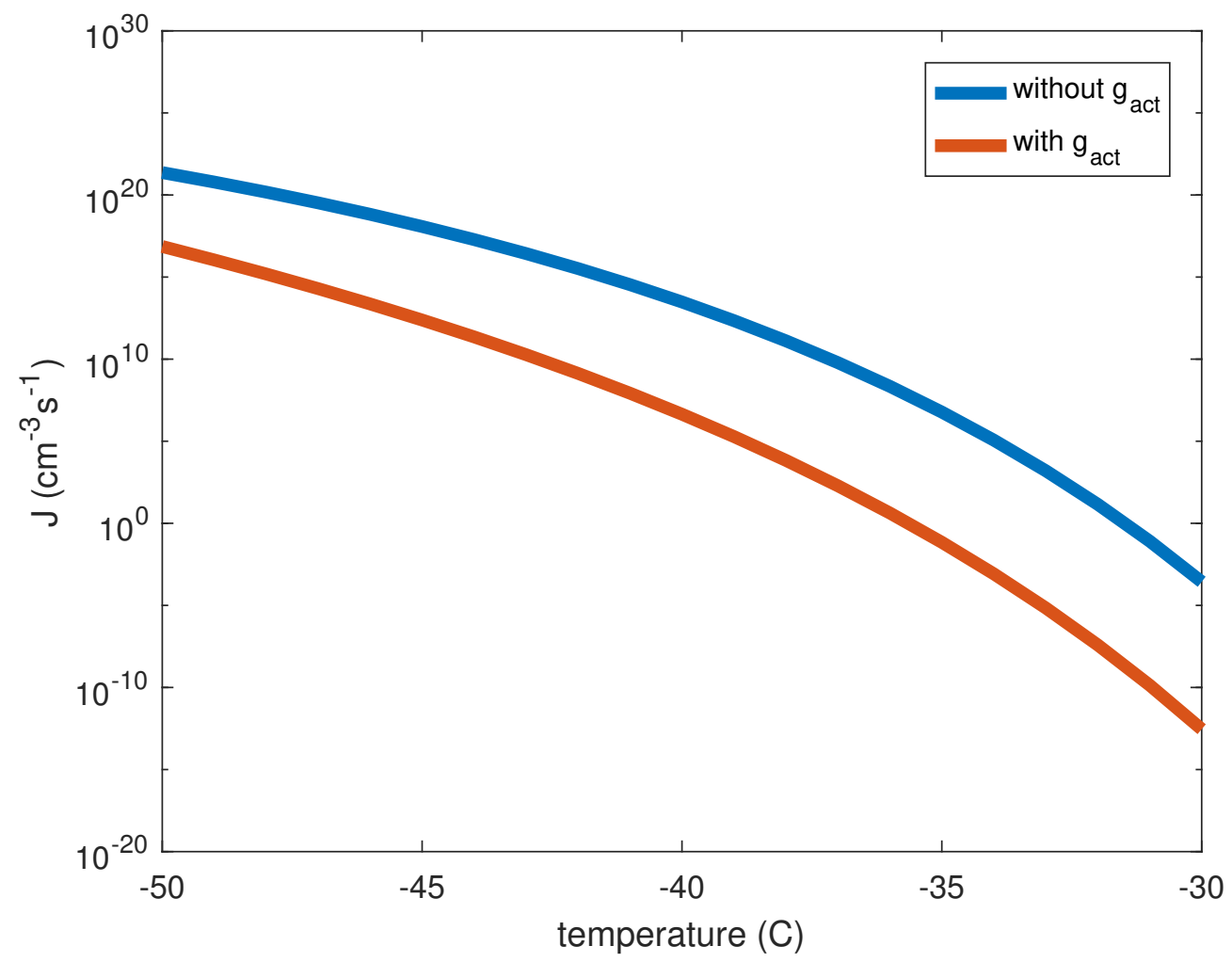

Figure 1.2: Homogeneous ice nucleation rate with and without $g_{a c t}$.

\section{Heterogeneous Ice Nucleation}

A foreign surface or particle (usually called an ice nucleus or an ice nucleating particle) can decrease the energy barrier for the formation of an ice embryo, thus increasing the ice nucleation rate. This type of ice nucleation is called heterogeneous ice nucleation. The critical energy barrier is

$$
\Delta G_{i}^{*}=\frac{16 \pi \sigma_{I L}^{3}}{3\left(n_{i} l_{f} \frac{\Delta T_{s}}{T_{0}}\right)^{2}} f_{h e t}
$$


where $f_{\text {het }}$ is a geometrical factor that depends on the interaction of supercooled water and ice with the heterogeneous nucleus. It should be mentioned that $f_{\text {het }}$ reduces the barrier but leaves the critical radius unchanged.

There are four modes of heterogeneous ice nucleation in the atmosphere: deposition, condensation, immersion and contact. Deposition mode occurs when water vapor is deposited on the ice nucleus and forms ice directly from the gas phase. Condensation mode occurs when an ice nucleating particle first serves as a cloud droplet nucleus and forms a supercooled droplet, and then triggers ice nucleation when the cloud droplet becomes larger (when the solute effect is small). Immersion mode occurs when an ice nucleating particle enters a cloud droplet before supercooling, and contact mode occurs when an ice nucleating particle collides with a supercooled droplet and triggers freezing. It is not easy to tell which ice nucleation mode contribute most in real clouds. Immersion and contact modes are believed to be the most important modes for ice production, according to some atmospheric models [45].

\section{Immersion Nucleation}

Because insoluble particles exist in cloud droplets, especially in polluted conditions, immersion ice nucleation is possible in the atmosphere, as long as the temperature 
is low enough. A wide variety of materials are known to serve as ice nucleating particles, such as soot, mineral dust, biological particles and secondary organic aerosol [46]. But not all of them are efficient ice nuclei. For more than half a century, experiments have investigated what types of materials act as good ice nucleating particles, meaning they are able to nucleate ice at relatively small supercooling temperatures. Instead of investigating the ice nucleation efficiencies for different types of particles, another research branch is to figure out what decides the ice nucleation efficiency for a given type of ice nuclei: chemical composition, geometric surface properties, or something else.

Although ice nucleation is a stochastic process (time dependent) from the molecular point of view, laboratory freezing experiments suggest that the temperature dependence is so strong that in many cases the time dependence can be neglected [46]. So why does a stochastic process exhibit aspects of a deterministic phenomenon? Niedermeier et al. assumed there are lots of active sites on ice nuclei, and different sites have different ice nucleation efficiencies [83]. If the number of active sites goes to infinity, that ice nucleus will exhibit singular behavior. Ervens and Feingold investigated classical ice nucleation theory and found that the ice nucleation rate is very sensitive to temperature, but very insensitive to time [28]. Westbrook and Illingworth claimed that the residence time for supercooled water is very short in most laboratory experiments, and stochastic ice nucleation might be very important for the long lived 
mixed phase clouds in the atmosphere [137]. The effect of this aspect of stochastic ice nucleation on the statistical properties of mixed-phase stratiform clouds will be further discussed in Chapters 4 and 5 .

\section{Contact Nucleation}

Previous results show that ice nucleation can occur at a higher temperature when an ice nucleus contacts a supercooled droplet than when an ice nucleus is embedded in it, no matter whether it is for water drops on a cold stage or freely suspended in air [36, 37, 38]. It is amazing that Gokhale and Lewinter used what for that time was a high speed camera (64 frames per second) to investigate the process of contact nucleation in early 1970s [36]. They found that an ice nucleating particle does not penetrate the surface of the supercooled water, but remains on it. The freezing is initiated at the point of contact. Freezing events initiated at multiple points are sometimes observed in their experiments. Since then, more experiments have been done to understand the mechanism of contact freezing [63, 84]. Several hypotheses have been proposed, including existence of ice embryos on impacting particles, reduction of the free-energy barrier for ice nucleation through impaction, role of the three phase contact line and propagation of pressure waves, but none of them have been confirmed [18, 34, 85, 109]. 
Research in our group is mainly focused on the three-phase contact line and the effect of line tension. Shaw et al. found that the freezing temperature is higher when the ice nucleating particle is near the surface than when it is immersed inside a supercooled water drop resting on a cold stage [111]. This is thought to be related to contact nucleation, even if the ice nucleus does not really have impaction motion [26]. The reason might be due to the effect of line tension [110]. Subsequent results show that in macroscopic systems the line tension does not play a role [40, 41], but there is indirect evidence that it may be important on nanotextured surfaces [39].

When we talk about ice nuclei, for either immersion or contact mode, they are typically considered to be all insoluble particles. However, contact ice nucleation can also be triggered by soluble particles. We know that salt particle can dissolve in water, and also suppress the freezing temperature. However, previous studies show that endothermic salts can enhance the freezing temperature of water through collision, and the mechanism is thought to be because of the cooling effect of salt dissolution [57, 58]. Recently, Niehaus and Cantrell found that even exothermic salts can trigger ice nucleation at higher temperature through collision [85]. They claimed that contact nucleation is due to pressure wave propagation in the supercooled water. Pressure might be an important factor for ice nucleation, which has been ignored for a long time in atmospheric sciences community. In the next section, the influence of pressure on the chemical potential driving a phase change will be introduced. 


\section{Pressure Induced Chemical Potential Change}

We begin by evaluating the chemical potential change at a given pressure $p^{\prime}$ and

temperature T. The derivation is an extension of those from prior studies [69, 82].

Defining $\mu\left(p_{0}\right)$ as the chemical potential of one phase at pressure $p_{0}$, then the chemical potential at another pressure $p^{\prime}$ is [94],

$$
\mu\left(p^{\prime}\right)=\mu\left(p_{0}\right)+\int_{p_{0}}^{p^{\prime}} \nu(p) d p
$$

Here $\nu(p)$ is the specific volume of that phase at pressure $p$. Assuming the water has a constant compressibility $\left(\nu_{l}\left(p^{\prime}\right)=\nu_{l}\left(p_{0}\right)\right)$, the chemical potential for water at $p^{\prime}$ and $\mathrm{T}$ is

$$
\mu_{l}\left(p^{\prime}\right)=\mu_{l}\left(p_{0}\right)+\left(p^{\prime}-p_{0}\right) \nu_{l}
$$

where the subscript $l$ refers to liquid water phase. Assuming ice is incompressible, the chemical potential for ice at $p^{\prime}$ and $\mathrm{T}$ is

$$
\mu_{s}\left(p^{\prime}\right)=\mu_{s}\left(p_{0}\right)+\left(p^{\prime}-p_{0}\right) \nu_{s}
$$


where the subscript $s$ refers to solid water phase. Therefore, the chemical potential change between supercooled water and ice at $p^{\prime}$ and $\mathrm{T}$ is

$$
\Delta \mu=l_{f} \frac{\Delta T}{T_{0}}+\Delta p \Delta \nu
$$

where $T_{0}$ is the equilibrium temperature between liquid water and ice, $\Delta T$ is the supercooling temperature, $\Delta p=p^{\prime}-p_{0}$ and $\Delta \nu=\nu_{l}-\nu_{s}$. It can be clearly seen that the first term on the right side is Equation [1.2. Because $\Delta \nu$ is negative for the water-ice system, the sign of $\Delta p$ determines whether pressure will increase or decrease the driving force of the phase change $\Delta \mu$, thus enhance or suppress the ice nucleation rate $J$. For example, the Laplace pressure of a nano-droplet is positive and non-negligible, and this might explain why nanoscale supercooled droplets can survive at very low temperatures without experiencing a phase change [69]. It has also been observed that due to the negative pressure occurring in a liquid capillary bridge, ice can form at a much higher temperature [90].

\section{Wetting Phenomenon}

When a droplet is placed on a substrate, the droplet might partially or totally spread on the substrate, which depends on the interaction between water and the substrate. 
The spreading parameter, which is the difference of the surface energy density between a dry and a wet substrate, is used to quantify the wetting phenomenon [22],

$$
S=E_{\text {substrate, } d r y}-E_{\text {substrate, wet }},
$$

or

$$
S=\sigma_{s o}-\left(\sigma+\sigma_{s l}\right)
$$

where $\sigma$ is the surface tension between water and air, $\sigma_{\text {so }}$ is the surface tension between substrate and air, and $\sigma_{s l}$ is the surface tension between water and substrate. If $S>0$, liquid can spread on, or wet, the substrate.

Usually, the drop will not totally wet the substrate $(S<0)$, instead it will form a spherical cap on the substrate with a contact angle $\theta_{E}$. The angle can be obtained from Young's relation,

$$
\sigma \cos \theta_{E}=\sigma_{s o}-\sigma_{s l}
$$

or

$$
\sigma \cos \theta_{E}=S+\sigma
$$

It should be mentioned that contact angle here is the macroscopic angle on an idealized substrate in a still environment. If the droplet is moving, the contact angle might be different when the droplet is moving forward (advancing contact angle) and backward (receding contact angle) due to surface pinning, which is called contact 
angle hysteresis. In addition, when close to the contact line, the contact angle at the nanometer scale might be different from the macroscopic contact angle. This is thought to be because of the line tension or local pinning [142].

\section{Electrowetting And Electrofreezing}

One way to distort the contact line, or change the contact angle of the droplet on a substrate is using the electrowetting technique. In fact, electrowetting can also be employed to study whether strong electric fields affect ice nucleation. This topic is relevant to experiments described in Chapter 2, and some previous studies in this field, and the basic phenomenon of electrowetting are discussed below.

Electric fields $(E)$ commonly exist in the atmosphere. $E$ is typically about $100 \mathrm{~V} / \mathrm{m}$ close to the surface in fair weather condition, and can be up to $10^{6} \mathrm{~V} / \mathrm{m}$ in a thunderstorm, until the dielectric breakdown of air. The origin of the strong electric fields occurring in cloud is believed to be related to the collision of ice particles. One interesting question is whether electric fields also can affect ice nucleation. This phenomenon is called electrofreezing, and has been studied for more than 50 years. There are two types of traditional experiment setups: parallel planes around a single droplet and cloud chambers containing a local voltage source, as shown in Figure 1.3, However, the results are quite confusing. Most chamber experiments indicate that electric fields 
can affect ice nucleation, while more careful parallel experiments show that electric fields have no effect on ice nucleation. [21, 24, 95, 97, 99, 104, 107, 112, 120, 121, 140]. However, results from molecular dynamics simulations show that electric fields can strongly enhance the ice nucleation temperature if the field can reach $10^{9} \mathrm{~V} / \mathrm{m}$ 122, 123, 144, 145, 146, 147, 156]. High electric fields can arrange the water molecules at the surface layer to a cubic-ice-like structure, thus triggering ice nucleation at a higher temperature than without the fields.
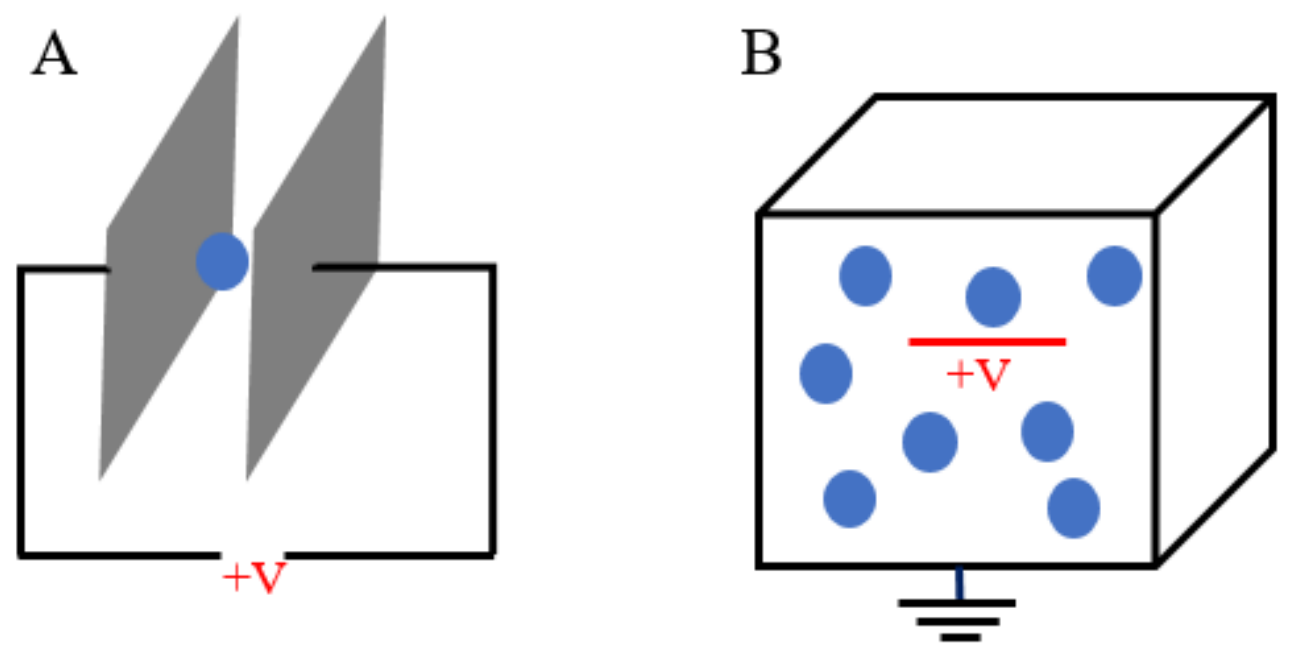

Figure 1.3: Sketch of two traditional electrofreezing setups: A is parallel plane electric field and B is high voltage wire inside of cloud chamber.

However, the electric field we can generate in the lab is usually limited by the dielectric breakdown of air. There is a huge gap between this field (about $10^{6} \mathrm{~V} / \mathrm{m}$ ) and the effective field claimed based on molecular dynamics simulations (about $10^{9}$ $V / m)$. So can we find a way to generate the electric fields larger than the dielectric 
strength of air? Yes, there is a way called electrowetting.

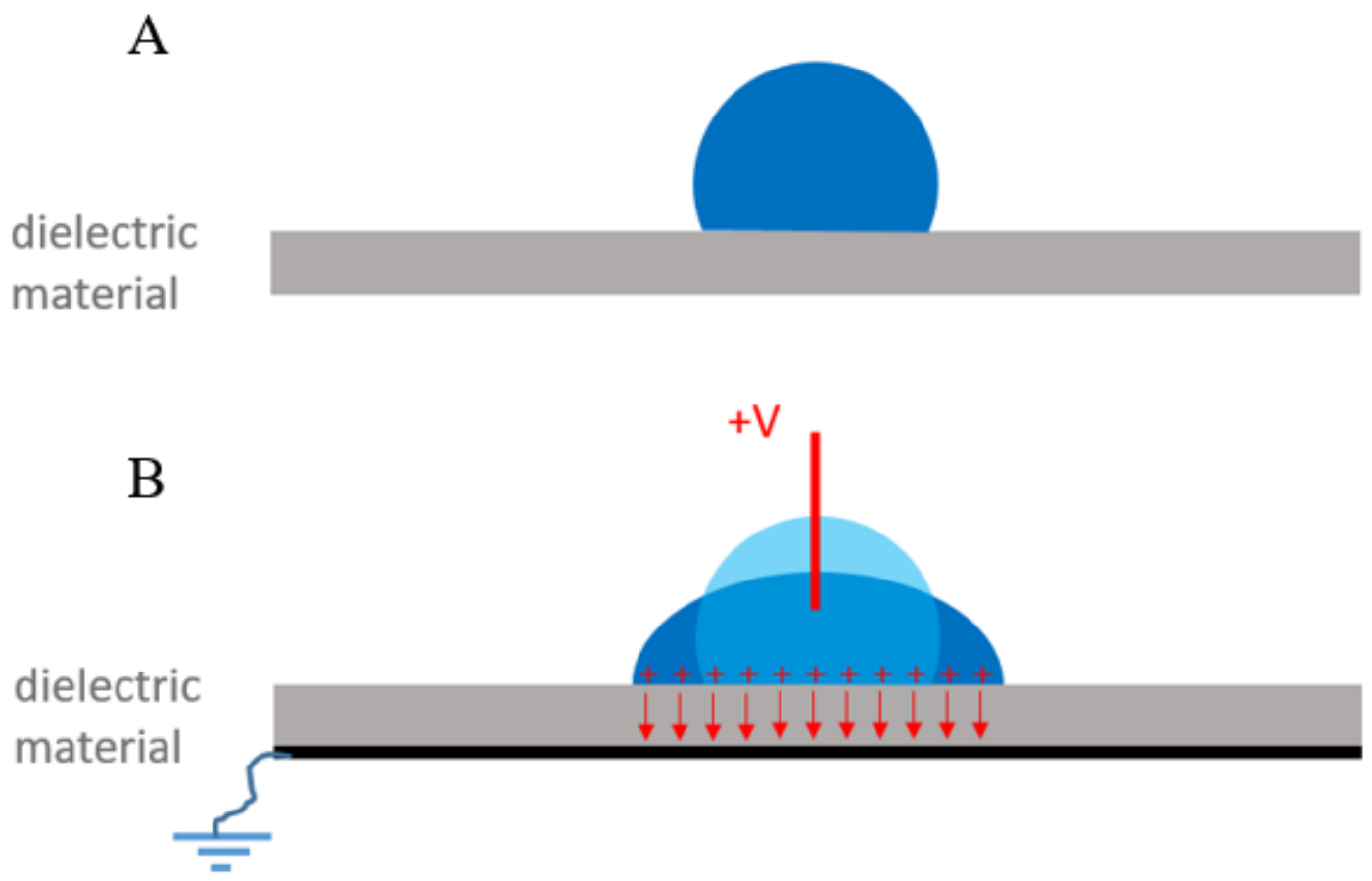

Figure 1.4: A) shows the water droplet on a dielectric substrate without an electric field. B) shows the standard setup for electrowetting, with a highvoltage electrode in the droplet and a grounded plane beneath the droplet.

Figure 1.4 shows the standard electrowetting setup. When the voltage is applied on an electrode in the droplet, a strong electric field will build within the dielectric layer, like a capacitor. This electric field is usually called the "electrowetting field". In Chapeter 3, we will discuss whether the electrowetting field is able to affect ice nucleation or not. In addition, the contact angle will decrease to lower the energy of the whole system. The contact angle change due to electrowetting is quantified by 
the Young-Lippmann equation [72],

$$
\cos \theta(U)=\cos \theta_{E}+\frac{\epsilon_{0} \epsilon_{1}}{2 d \sigma} U^{2}
$$

where $U$ is the applied voltage, $d$ is the thickness of the dielectric substrate, and $\epsilon_{1}$ is the dielectric constant of the liquid. This equation only works when the contact angle is large, e.g. on a hydrophobic substrate. If the contact angle is very small, the applied voltage cannot decrease the contact angle any more, which is called the saturation phenomenon [81].

\section{Droplet Vibration On A Vertically-Oscillating}

\section{Plane}

Another way to move the contact line is by setting a droplet on a vertically-oscillating plane. In Chapter 4, we will discuss whether the resulting vibrational motion affects ice nucleation. Here we will first introduce basic properties of water droplets on a solid plane, and the response of water droplets on an oscillating plane.

The response of sessile droplets on a vertically-oscillating plane has been well studied 
[87, 88]. Noblin et al. found that a resonant frequency of the sessile droplet is,

$$
\omega_{j}^{2}=\left(g q_{j}+\frac{\sigma}{\rho} q_{j}^{3}\right) \tanh \left(q_{j} \frac{V}{\pi R^{2}}\right)
$$

where $q_{j}$ is the wavenumber for mode $j, \sigma$ is surface tension, $\rho$ is water density, $V$ is the volume of the droplet and $R$ is the radius of the wetting area.

They also found that two types of oscillations for sessile droplets occur on a plane undergoing vertical oscillation at constant frequency [87]: (1) at small amplitude, the contact line is pinned and the contact angle oscillates, a phenomenon known as contact angle hysteresis; (2) at high amplitude, the contact line becomes depinned and is able to move. Basically, there is a critical amplitude for each substrate, below which the contact line cannot move, and above which the contact line is able to move. In Chapter 3, this contact angle hysteresis will be an important aspect of the experiments. 


\section{Chapter 2}

\section{Ice Nucleation At The Contact}

\section{Line Triggered By Transient}

\section{Electrowetting Fields}

This chapter details the ice nucleation experiment with a transient electrowetting

fields 1 1 . This work was based on a research collaboration and is published in full form in the Applied Physics Letters [152], and is reprinted with permission by the American Institute of Physics2.

\footnotetext{
${ }^{1}$ This paper is co-authored by R.A. Shaw, C.W. Gurganus, S.K. Chong and Y.K. Yap ${ }^{2}$ Reprinted with permision from: F. Yang, R.A. Shaw, C.W. Gurganus, S.K. Chong, and Y.K. Yap, Applied Physics Letters, 107, 264101, 2015. Copyright 2015 by American Institute of Physics.
} 


\section{Abstract}

Supercooled water is found to have a significantly enhanced freezing temperature during transient electrowetting with electric fields of order $1 \mathrm{~V} / \mathrm{m}$. High speed imaging reveals that the nucleation occurs randomly at the three-phase contact line (droplet perimeter) and can occur at multiple points during one freezing event. Possible nucleation mechanisms are explored by testing various substrate geometries and materials. Results demonstrate that electric field alone has no detectable effect on ice nucleation, but the moving boundary of the droplet on the substrate due to electrowetting, is associated with the triggering of nucleation at a much higher temperature.

\section{Introduction}

Nucleation of a solid from a liquid is a problem of broad relevance in many natural systems and technological applications [110]. For example, the nucleation of ice from supercooled liquid water is a critical step in the chain of events leading to precipitation

formation in many clouds [11]. Indeed, nucleation of ice is particularly enigmatic and is the subject of active research [68, 85, 86]. This chapter describes experiments that touch on two aspects of liquid-solid nucleation in general, and water-to-ice nucleation in particular: the influence of an external electric field [35, 74, 110] and the possibility 
of preferred crystallization at liquid surfaces or, when a foreign substrate is present, at the three-phase contact line [39, 70].

Early cold stage experiments showed that supercooled water droplets can freeze when an electric field is applied [106]. Since that time, various experiments with bulk water and dispersed water droplets in a supercooled state, with electric field strengths up to approximately $0.1 \mu m$ have given conflicting results [24, 97, 99, 104, 107, 120, 134, 140]. And yet under some experimental conditions, remarkable electrofreezing of water has been observed [9]. Molecular dynamics simulations suggest that external electric fields significantly promote both homogeneous and heterogeneous ice nucleation when the field strength is larger than $1000 \mu \mathrm{m}$ [122, 144, 146]. It is believed that in the high electric field, locally polarized liquid can decrease the critical size of nucleus, thus facilitating ice nucleation. However such high fields are difficult to achieve in reality because of electric breakdown. Recently, Carpenter and Bahadur generated ultrahigh electric fields up to $80 \mu \mathrm{m}$ using thin dielectric films in an electrowetting geometry [101], and found that interfacial electric fields alone can significantly elevate freezing temperatures by more than 15 degrees [12]. These results are consistent with findings from other substances, in which field strengths of 100 to $1000 \mathrm{~V} / \mu \mathrm{m}$ are observed to enhance nucleation rate [74, 110].

Pruppacher was apparently the first to note that nucleation induced by an electric 
field has a tendency to initiate from the contact line formed at a substrate (airwater-substrate line) [97, 99]. Since then, similar observations have been reported for freezing in the presence of electric fields [12, 27]. Given our groups interest in contact freezing [39, 40, 41, 111], we were motivated by these recent studies to further investigate the role of the contact line in ice nucleation induced by electric fields.

\section{Experimental Methods}

Our experiments used a simple electrowetting setup: a single water droplet resting on an electrically insulating substrate, the droplet in contact with a metal electrode and the substrate resting on a conducting plate (Figure 2.1 a). Those components sit on a $10 \mathrm{~mm}$, temperature-controlled copper block at the center of an insulated chamber. Freezing is recorded from above using a high-speed camera (Photron SA6) at 5000 frames per second and with an effective pixel size of approximately $26.3 \mu \mathrm{m}$. The overhead camera view allows observation of both the contact-line movement during electrowetting, and the point of initiation of freezing. This system has been used previously to study the freezing of drops on silicon substrates, and results show that there is no preference for freezing at the contact line unless the surface is nanotextured [39, 40, 41]. In this study, a thin polymer film (McMaster-Carr, Kapton Polyimide Film, $25 \mu m$ thick) was placed on the copper block to isolate the substrate above. A grounded smooth silicon wafer (Wafer World, Inc., test grade, 250 - $300 \mu m$ thick) 
rests on the polyimide film to serve as one electrode. Although silicon is a semiconductor, results shown that there is no significant difference if we use a grounded copper substrate instead (the silicon substrate provides better visualization from above due to the dark background). A 0.22-mm-thick siliconized glass slide (Hampton Research Corp., HR8-082) is used as the droplet substrate. The substrate was washed with acetone, alcohol, distilled water, and dried with a clean air flow. A $20 \mu L$ droplet of type 1 grade water (distilled, deionized, UVirradiated) was put on the glass cover. A flow of filtered dry air $\left(2 \mathrm{~L} \mathrm{~min}^{-1}\right)$ is fed into the chamber to decrease the inside relative humidity and make sure no dew or frost can form on the substrate at temperatures above $20{ }^{\circ} \mathrm{C}$. The air in the chamber is therefore subsaturated and the droplet slowly evaporates, but this does affect the ice nucleation efficiency significatly [41]. A rigid piano wire is connected to a DC power supply and a voltage up to $2000 \mathrm{~V}$ is applied, corresponding to an electric field up to $10 \mathrm{~V} / \mu \mathrm{m}$. The horizontal position of the tip can be controlled by a piezoelectric translation stage. An image of a water droplet taken with the high speed camera is shown in Fig. 1b in the main text. The horizontal position of the tip can be controlled by a piezoelectric translation stage. An image of a water droplet taken with the high speed camera is shown in Figure 2.1 b. 


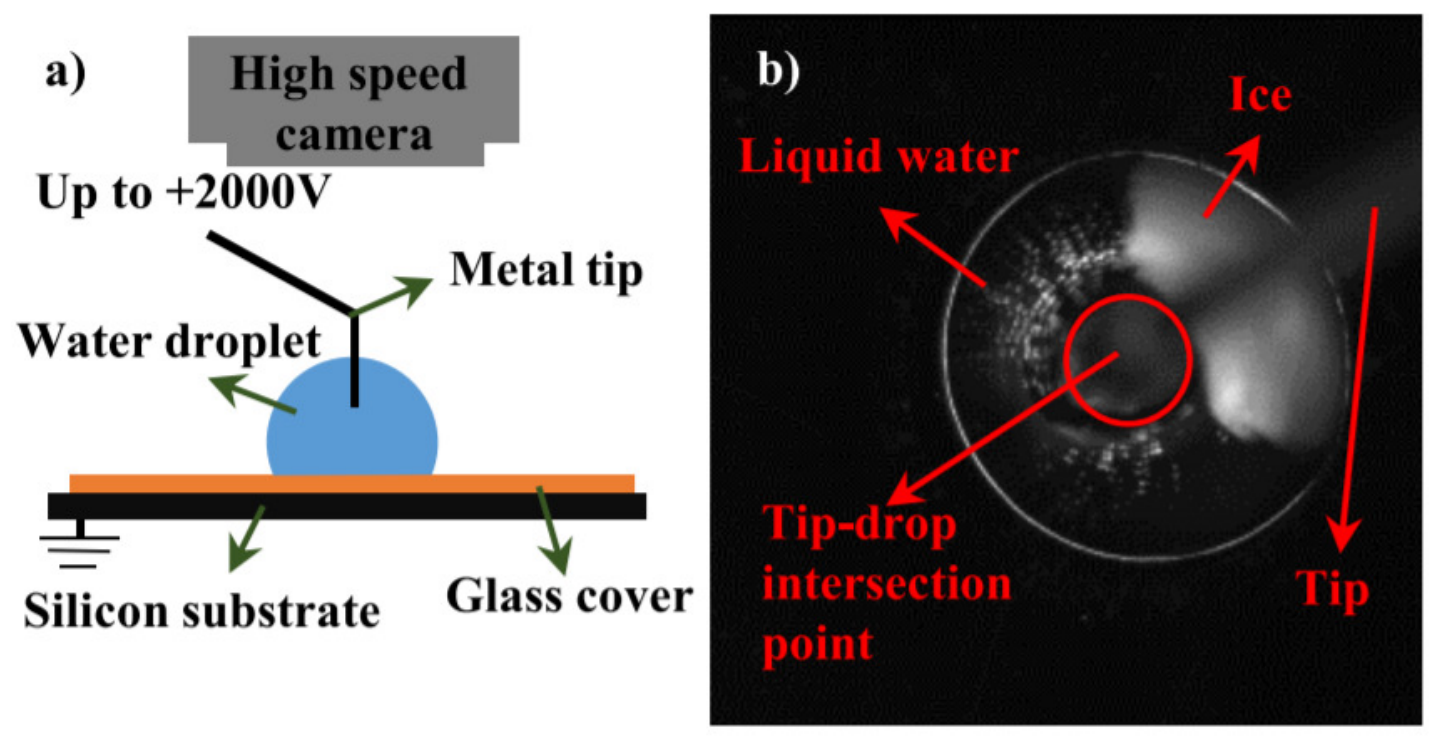

Figure 2.1: a) Sketch of the experimental setup from the side, illustrating the electrowetting geometry. b) Top view of a crystalizing droplet from the high speed camera.

\section{Results and Discussion}

As a control experiment, a $20 \mu L$ droplet rests on the silica glass with no voltage applied; the freezing temperature is observed to be $-24.7 \pm 0.7^{\circ} \mathrm{C}$ for a $2.0^{\circ} \mathrm{C} / \mathrm{min}$ cooling rate (All experiments are repeated ten times for statistical significance.) Without the electric field, the freezing is always initiated from a single point, randomly distributed on the immersed substrate (not at the electrode, which means the electrode is not a good ice nucleation agent compared with the substrate.). To investigate the role of the electric field we applied three voltages $(600 \mathrm{~V}, 800 \mathrm{~V}$ and $1000 \mathrm{~V}$ ) between the electrode and the silicon wafer; the voltage was applied with the droplet 
above $0{ }^{\circ} \mathrm{C}$ and then the temperature was decreased at $2 \mathrm{~K} / \mathrm{min}$. The mean freezing temperatures were $-23.7 \pm 0.7{ }^{\circ} \mathrm{C},-23.3 \pm 2.4{ }^{\circ} \mathrm{C}$ and $-23.2 \pm 1.6{ }^{\circ} \mathrm{C}$ for $600 \mathrm{~V}, 800$ $V$ and $1000 V$ respectively. Results show that the mean freezing temperature slightly increases as the voltage increases, but not significantly, and freezing temperatures were always lower than $-20^{\circ} \mathrm{C}$. Electric fields for the three voltages are 2.7, 3.6, and

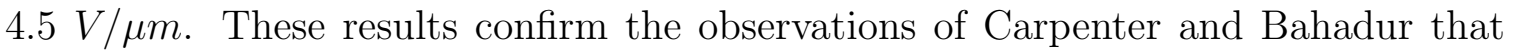
electric fields smaller than $5 \mathrm{~V} / \mu \mathrm{m}$ have a small effect on ice nucleation [12].

However, the observation changes dramatically if we first cool down the temperature to a value above $-20{ }^{\circ} \mathrm{C}$, maintain at least $5 \mathrm{~min}$ to ensure no freezing occurs, then turn on the field. In this scenario, ice nucleation is triggered even at much higher temperatures. Experiments were done between $-3{ }^{\circ} \mathrm{C}$ and $-10^{\circ} \mathrm{C}$ for the same three voltages. Each case is repeated ten times, and the observed freezing probability is shown in Figure 2.2. It can be seen that freezing fraction increases with increasing voltage and with decreasing temperature. It reaches $100 \%$ at $-7{ }^{\circ} \mathrm{C}$ for $1000 \mathrm{~V}$, at $-9{ }^{\circ} \mathrm{C}$ for $800 \mathrm{~V}$, and nearly $100 \%$ at $-10{ }^{\circ} \mathrm{C}$ for $600 \mathrm{~V}$ (only one out of ten doesnt freeze). From these observations we conclude that electric field alone cant be the reason for this high temperature freezing behavior. At these temperatures and with a static electric field switched on above $0{ }^{\circ} C$, the supercooled droplets can be held for very long time without freezing.

The observation is more surprising if we turn on the field with the droplet above 0 
${ }^{\circ} \mathrm{C}$, and then cool it down to a value above $-20{ }^{\circ} \mathrm{C}$ and maintain at least $5 \mathrm{~min}$ to make sure no freezing occurs; then when the field is switched off there still exists a high probability for the droplet to freeze, especially for higher voltage. The freezing fractions for switching off $600 \mathrm{~V}, 800 \mathrm{~V}$ and $1000 \mathrm{~V}$ in droplets at a range of temperatures $\left(-4{ }^{\circ} \mathrm{C}\right.$ to $\left.-10^{\circ} \mathrm{C}\right)$ are shown in Figure 2.2. Although the freezing fraction for turning off the voltage is usually smaller than that for turning on at the same temperature, it is striking to us that ice nucleation is triggered with $100 \%$ probability by turning off the $1000 \mathrm{~V}$ voltage for temperatures equal or below $-8^{\circ} \mathrm{C}$.

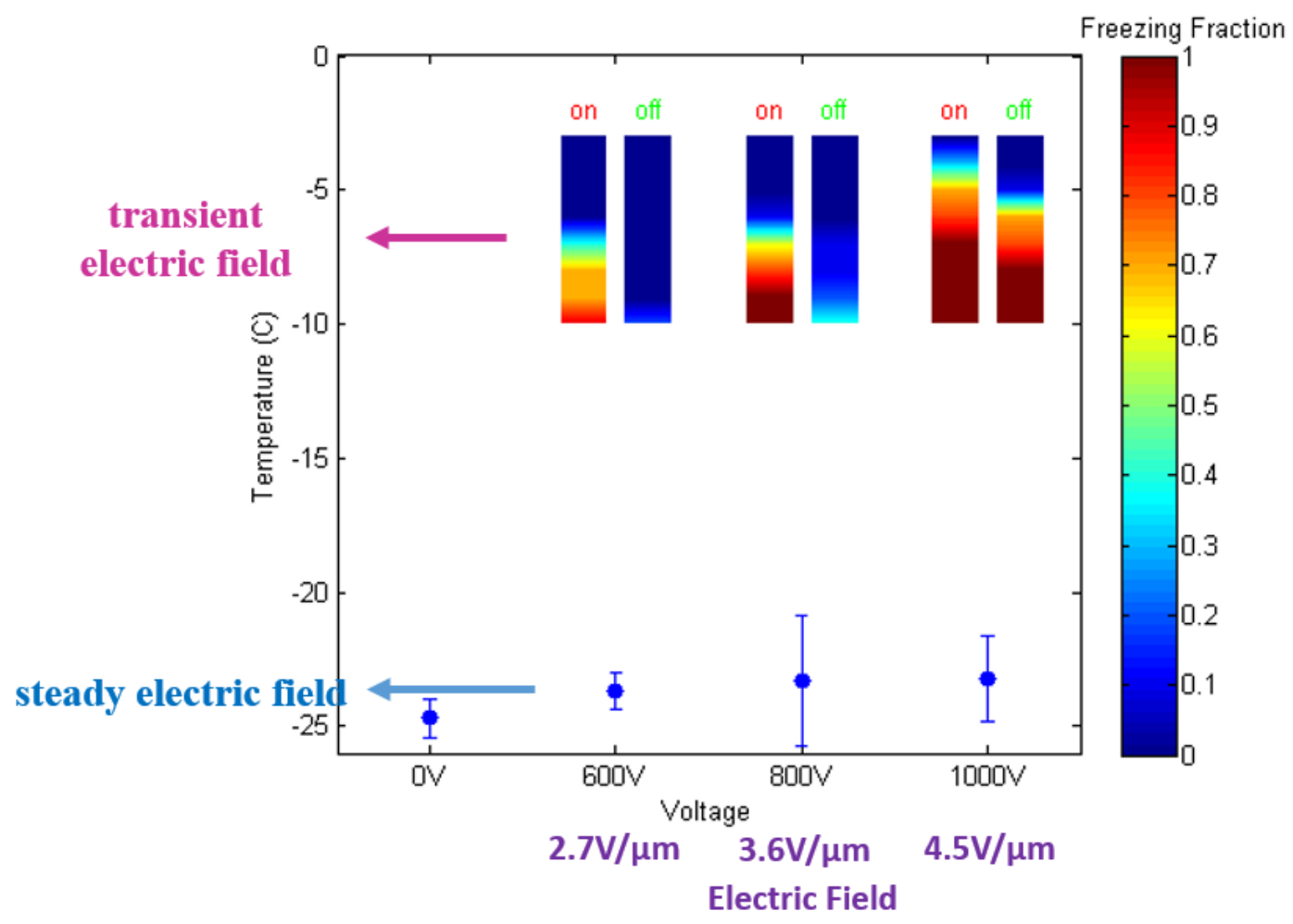

Figure 2.2: Natural freezing temperature for different voltages (blue points) and freezing fraction when turning on/off the electric field at a constant temperature. 
With the $5 \mathrm{kHz}$ high-speed camera we find three interesting things about the ice nucleation that occurs when an electric field is turned on. First, when we turn on the field, the droplet will shake and its boundary will expand due to the decrease of contact angle associated with electrowetting [101]. Figure 2.3 shows examples of time-resolved images from the high speed camera when turning on the voltage at -10 ${ }^{\circ} \mathrm{C}$ and $-15^{\circ} \mathrm{C}$. Boundary movement is more significant at $1000 \mathrm{~V}$, as expected for electrowetting: larger voltage leads to a smaller contact angle. In addition, boundary movement is more obvious at $-10{ }^{\circ} \mathrm{C}$ compared with $-15^{\circ} \mathrm{C}$. This is because the ice propagation speed is faster at lower temperature, so once the edge freezes, it cannot move any more.

The observed time for boundary expansion is about $20 \mathrm{~ms}$ before we can detect the initial droplet freezing at $10 \mathrm{C}$, and $4 \mathrm{~ms}$ at $15 \mathrm{C}$. The boundary movement is related to the dynamic process of electrowetting [19]. Based on Quilliet and Berge (2001), the charge density is constant far from the wetting line, but a few percent larger around the boundary due to edge effect [101]. From thermodynamics point of view, the expanding boundary is a result of minimizing the total free energy of the system. From electromechanical point of view, the boundary movement is driven by the electric force on the droplet due to surface charge. More details can be seen in a review paper by Mugele and Baret (2005) [81]. In addition, we find that the expanding velocity of the triple line is not uniform: the triple line is disturbed and the moving boundary has a wave like shape. We did find the local radius of curvature to be on 
the order of few $10 \mathrm{~m}$, when the triple line is moving. As far as we know, nobody has studied this non-uniform expanding velocity of triple line during the dynamic spreading process. We believed that the wave like shape might be due to the pinning effect, or the Rayleigh charge instability [23].

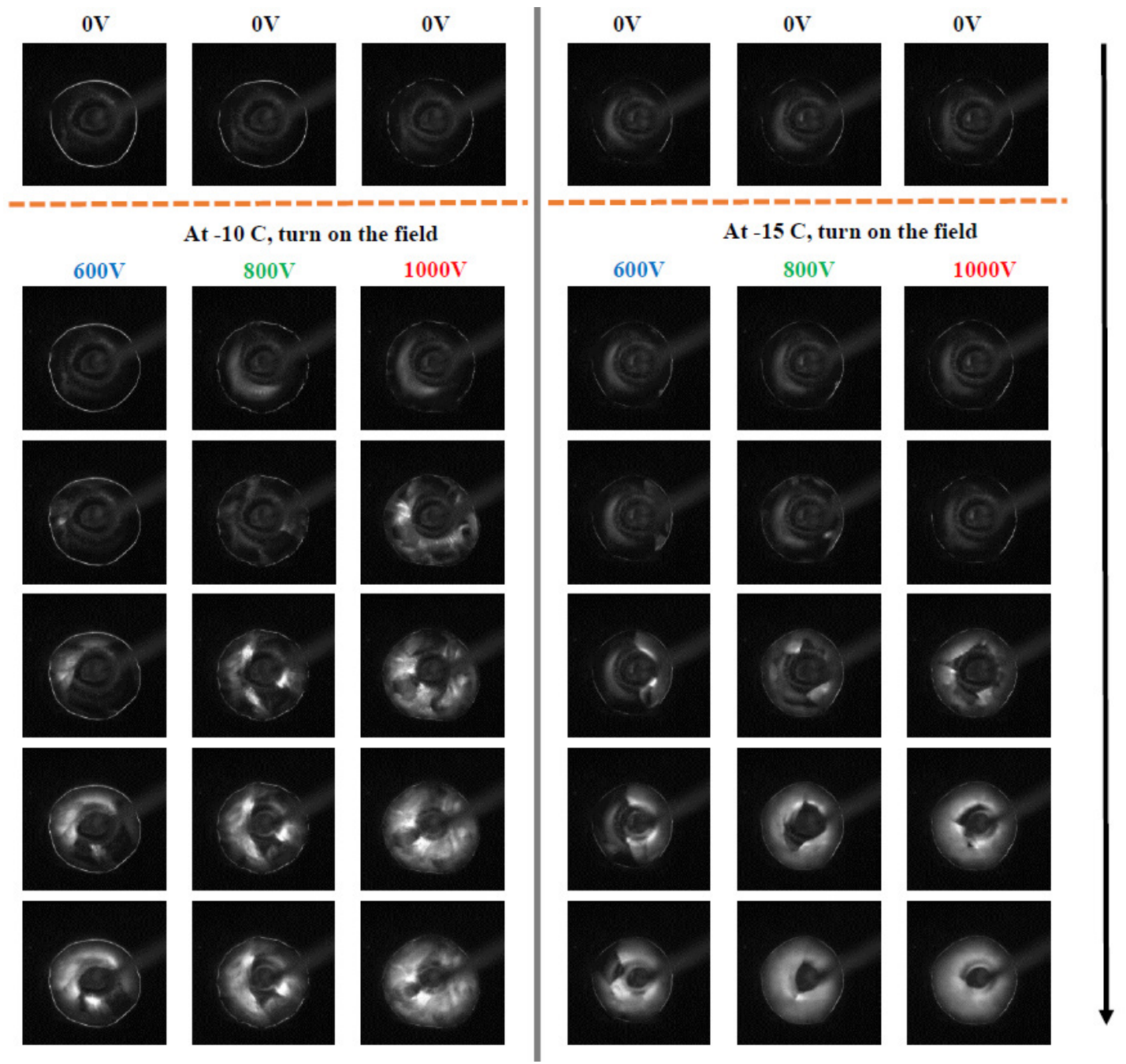

Figure 2.3: Time-lapse views of crystallization after switching on three voltages $(600 \mathrm{~V}, 800 \mathrm{~V}$ and $1000 \mathrm{~V})$ at $-10{ }^{\circ} \mathrm{C}$ (left) and $-15{ }^{\circ} \mathrm{C}$ (right). The images are taken with a $5 \mathrm{kHz}$ high speed camera. Each frame in one column is separated by $10 \mathrm{~ms}$. 
The second interesting observation is that ice always nucleates at the three-phase contact (triple) line, as shown in Figure 2.3. From this we expect that the nucleation mechanism is unlikely due to the changing of the surface charge density because the charge concentration at the edge is only a few percent larger than inside the drop [101]. If the charge concentration can affect ice nucleation, we might reasonably expect that as we increases the voltage we should also see nucleation start away from the triple line; but ice always forms from the edge even for voltages up to $2000 \mathrm{~V}$. This is consistent with previous finding that surface charge doesnt affect ice nucleation [105].

Thirdly, the nucleation sites are randomly distributed along the triple line, and there can be multiple nucleation sites, especially for high voltage. Figure 2.3 shows that nucleation starts all around the edge when switching on $1000 \mathrm{~V}$. This is significantly different compared with cooling down the droplet without the electric field, or applying the field above $0{ }^{\circ} \mathrm{C}$ then cooling down the droplet. Under those conditions, the nucleation site is only single point. This implies that the nucleation rate on the edge is extremely large when we turn on the field (waiting time for nucleation events along the perimeter is less than the time for droplet crystallization).

For ice nucleation when turning off the voltage, we still see a slight deformation of the droplet, but not as obvious compared with that when turning on the voltage. This is referred to as the reversibility problem in electrowetting [155]. When turning off the voltage nucleation usually occurs only at one point, and randomly located around 
the edge. This is quite different compared with ice nucleation when turning on the voltage, which is usually multiple points or even all around the droplets.

From these observations we conclude that the nucleation mechanism for turning on/off the field is unlikely due to the electric field alone, and also is unlikely due to the change of surface charge density. It is more likely that this nucleation is related to the movement of the three-phase contact line. So what is the possible nucleation mechanism? Possibilities include the existence of frost nearby on the substrate, a substrate-specific property, the dynamic boundary movement alone, or the existence of locally high electric fields at the droplet edge. We investigate these possibilities with several additional experiments.

\section{(a) Is there frost on the substrate nearby the droplet?}

If so, when the triple line expands due to electrowetting, it might touch the frost and freeze the whole droplet. However, this possibility is ruled out by two experiments: 1) we first cool down the temperature to $-15^{\circ} \mathrm{C}$ or example, and maintain 5 min, with no freezing occuring. Then we use the electrode tip (mounted on a piezoelectric translation stage) to drag the droplet across the glass cover. No freezing occurs whether we pull or push the droplet, although the displacement is much larger than the boundary movement due to the electrowetting. This experiment also proves that mechanical movement alone cannot trigger ice nucleation. 2) we surround the droplet with pump oil (Hyvac products, Inc ). Although the air-water-substrate triple line 
changes to an oil-water-substrate triple line, the contact nucleation efficiency should not be strongly affected [111]. In this way, no frost can form nearby the droplet due to the oil isolation. However, we can still trigger ice nucleation when we apply 1000 $V$ at $10^{\circ} \mathrm{C}$.

\section{(b) Is there a dependence on the substrate?}

To test this, we applied voltages up to $2000 \mathrm{~V}$ at $-10{ }^{\circ} \mathrm{C}$ on various substrates: 0.96 $m m$ siliconized glass (Hampton HR3-247), $25 \mu m$ polyimide film (McMaster-Carr Kapton Film, 2271K1), $1.0 \mathrm{~mm}$ plain glass (Fisherbrand Plain Microscope slides, 12-549-3), and $25 \mu \mathrm{m}$ mica sheet (Tarheel Mica Co.). Results are shown in Table 1. We can trigger ice nucleation on both thick siliconized glass and thin polyimide film, but not on plain glass and mica sheet. However, if we immerse the droplet in oil, we can also trigger ice nucleation on plain glass and mica sheet. With the high speed camera, we find that the droplet only freezes when the boundary is observed to expand when we turn on the field. We can see the boundary movement when we apply the voltage on siliconized glass, polyimide film, plain glass with oil surrounded the droplet and mica sheet with oil surrounded the droplet, but we cannot see any movement on plain glass and mica sheet with air surrounded even for voltages up to $2000 \mathrm{~V}$. This phenomenon appears to be related to contact angle saturation in electrowetting [81, 128, 129]. For plain glass and mica sheet, the water-air contact angles are $9^{\circ}$ and $26^{\circ}$ separately. The contact angle is sufficiently small that it may 
already be saturated or does not change significantly when we apply the voltage. But the water-oil contact angles on both substrates are larger than $40^{\circ}$. In this case, electrowetting can decrease the contact angle efficiently, and thus the boundary will expand. Another possible explanation is that for clean mica, the substrate is wet by a molecularly thin water layer (e.g., pseudo partial wetting) [23]. Therefore, there might be no three phase contact line and strictly a contact angle does not exist [66]. This might explain the absence of boundary movement on the substrate.

From above we conclude that this freezing phenomenon is related to boundary movement associated with electrowetting. It can occur on different substrates, as long as the contact angle is large enough that electrowetting can affect it. In addition, because the mica sheet is atomically smooth compared with glass or polyimide film, the freezing observed on mica sheet rules out the possibility that nanoscale texture might cause a higher freezing temperature at the three-phase contact line [39].

\section{(c) What are relative roles of triple-line movement and the changing elec- tric field?}

From the experiments described thus far we know that macroscopic boundary movement alone cant trigger ice nucleation, but boundary movement due to electrowetting is related to the ice nucleation. To test the relative roles of the triple-line movement and the changing electric field, we modify the glass substrate with a graphene layer and a polymer ring. 
Graphene sheet was synthesized on a $\mathrm{Cu}$ substrate using a facile chemical vapor deposition (CVD) technique. The $\mathrm{Cu}$ substrate was soaked in acetic acid for 15 minutes to remove the native oxide of $\mathrm{Cu}$, and then rinsed with deionized water to wash away the acid. The cleaned $\mathrm{Cu}$ substrate was placed into a closed end quartz tube, which was then inserted into a vacuum CVD reactor. A hydrogen treatment was performed on the $\mathrm{Cu}$ substrate at a temperature of $1000{ }^{\circ} \mathrm{C}$ in a constant $H_{2}$ flow rate of $100 \mathrm{sccm}$ for 30 minutes. The temperature of the furnace was then increased to $1100{ }^{\circ} \mathrm{C}$. As the furnace reached the steady temperature, $10 \mathrm{sccm}$ of methane and 300 sccm of argon gases were introduced into the furnace for the graphene growth. After 10 minutes of graphene growth, the reactor was turned off and the sample was cooled down in the flow of argon gas. The as-grown graphene sample was characterized using a field emission scanning electron microscopy (Hitachi S-4700 FESEM) and microRaman spectroscopy (JobinYvon LabRAM HR800 spectrometer). FESEM image shows the growth of a nearly continuous graphene sheet. Micro-Raman spectrum confirms the graphene sheet on the $\mathrm{Cu}$ substrate. The thickness of the graphene sheet estimated from the full width at half maximum of the graphene Raman 2D band is 2-3 layers. The graphene sheet was transferred onto a siliconized glass substrate via a chemical etching and transfer process. A thin layer of PMMA was spin coated on the graphene/Cu sample. The PMMA/graphene/Cu sample was immersed in a concentrated iron chloride electrolyte to etch away the $\mathrm{Cu}$ substrate. The remaining PMMA/graphene was rinsed with deionized water and transferred to a siliconized 
glass substrate. The PMMA coating was removed by heating up the sample at 160 ${ }^{\circ} \mathrm{C}$ using a hot-plate.

Three geometric graphene layers are transferred on the glass cover for comparison: a fully graphene covered glass slide, a half graphene covered glass slide, and a graphene ring with the glass slide exposed in the center. Because graphene is a good conductor, no electric field exists at the graphene-water interface, and we therefore do not expect to see freezing start from the graphene substrate. In a last test to explore the possible role of triple-line movement, we constructed a round polymer 'wall' on the glass substrate (using oven-dried glue). The polymer acts as a stiff wall so that the waterglass-polymer triple-line cannot move.

Results show that for the full graphene covered glass substrate, graphene ring with exposed glass in the center, and glass substrate with polymer wall, no boundary movement was observed and freezing did not occur, even for voltages up to 2000 $V$. No freezing on the graphene ring and the polymer wall substrate indicates that the changing electric field alone without the boundary movement cannot trigger ice nucleation. For the half graphene, half glass substrate, the droplet was observed to freeze when the voltage was switched on. We also observed triple-line movement and nucleation sites, all confined to the glass side.

Several additional notes should be mentioned: No changes in results were observed when the direction of the electric field was reversed (negative voltage applied to 
droplet). There is no steady electric current in the water, although a charging current exists when we switch the field on or off. However, electrolysis is unlikely to occur during this process because we did not observe bubbles, and nucleation was not observed at the electrode tip as would be expected [47, 112, 113]. Furthermore, no nucleation was observed when a current was run through the droplet on a conducting substrate. Finally, no electrical breakdown was observed.

Our experiments show that ice nucleation probability is strongly enhanced during transient electrowetting. The observed freezing temperature is much higher than that for a static electric field. High speed camera images reveal three phenomena that occur when electric field is switched on: (1) the droplet expands due to electrowetting; (2) nucleation sites are always randomly located around the droplet three-phase contact line; and (3) nucleation occurs at multiple points, especially for higher voltage. To understand the nucleation mechanism, we do experiments on various substrates. Results indicate that this freezing is not a result of macroscopic boundary movement without the electric field (droplet dragged by electrode); or the electric field alone; or the change of electric field alone without triple-line movement; or the transient charging electric current. The nucleation must be related to the boundary movement resulting from electrowetting. One possibility is that locally-high electric fields may be formed at the distorted boundary during the transient electrowetting process, leading to electrofreezing [122, 144, 146]. Alternatively, ice nucleation may be due to the combination of boundary movement and high electric field. Simulations 
have shown that oscillatory shear in combination with a static electric field proved to be much more efficient in crystallization than an electric field alone.39 But both of these possibilities must face our observation that freezing occurs even when the the electrowetting field is switched off. The exact mechanism remains unknown, but the observations clearly implicate the triple line, and therefore suggest a link to the phenomenon of contact nucleation in the atmosphere [38].

\section{Acknowledgement}

A special thanks goes to Adam Laxo (MTU Physics), who helped with earlier experiments on electrofreezing, and to Weilue He (MTU Biomedical Engineering), who helped in designing the glass substrate with glue wall. This research was supported by the DOE Office of Science as part of the Atmospheric System Research program through grant DE-SC0006949. Y.K.Y acknowledges support from National Science Foundation, Division of Materials Research (Award Number 1261910). 


\section{Chapter 3}

\section{Non-thermal Ice Nucleation}

\section{Observed At Distorted Contact}

\section{Lines Of Supercooled Water Drops}

This chapter details the ice nucleation experiment on a vertical vibrational plane.

This work was based on a research collaboration and is just submitted. 


\section{Abstract}

Conditions under which supercooled water freezes are not only crucial for determining the precipitation efficiency of clouds and the climatalogically-significant vertical profile of water within the earth's atmosphere, but also underlie fields as diverse as evolution in extreme environments, food preservation, and the design of anti-icing surfaces [5, 11, 62, 67, 103, 110, 141]. Most work has focused on the roles of temperature, supersaturation and material properties[2, 46], while little attention has been devoted to dynamic effects, although it has long been noted that supercooled water can freeze instantly by shaking or tapping[25]. In this work, carried out at constant temperature, we explore nucleation of ice in supercooled water initiated by non-thermal means. Here we show that mechanical agitation induces freezing of supercooled water drops at distorted contact lines, pointing to negative pressure perturbation, rather than temperature, as a cause of ice nucleation. Our nucleation rate calculations show that, given the water density anomaly, plausible negative pressures $\left(\sim 10^{8} \mathrm{~Pa}\right)$ can account for the observed increase in freezing temperature $(\sim 10$ $\mathrm{K}$ ). The notion that negative pressure can trigger water-ice nucleation, provides a new context for interpreting long-mysterious observations, such as the surprising efficiency of contact nucleation in clouds, in terms of contact-line distortion. The results therefore suggest a need to explore what range of pressure perturbations occur in agitated supercooled cloud droplets, such that they can be included in atmospheric 
ice nucleation models.

\section{Introduction}

The phenomenon of contact nucleation comes from the observation that supercooled water droplets freeze at a higher temperature when a particle impacts the water surface, compared to the particle being immersed in the droplet [64]. Several possible mechanisms have been proposed, including existence of ice embryos on impacting particles, reduction of free-energy barrier for ice nucleation through impaction, role of line tension and propagation of pressure waves [18, 34, 39, 85]. However, why dynamic impaction will enhance ice nucleation is still far from clear. Recently, we found that a moving contact line due to electrowetting can strongly enhance the rate of ice

nucleation[152]. A mechanism remained elusive, however, thereby motivating further investigation of the influence of a moving contact line in the absence of an electric field. In the experiments presented here, we observe a single water drop resting on a substrate subjected to vertical oscillations. Non-thermal contributions to the nucleation process are considered by performing the experiments at a constant temperature significantly higher than the natural freezing temperature of the substrate. Further insight into the mechanism is obtained by pinpointing the location of freezing of the supercooled drop with high-speed imagery. 


\section{Experimental Methods}

The freezing experiments were carried out inside an insulated, isothermal container (Engel MHD13F-DM), with controllable temperature down to $-18{ }^{\circ} \mathrm{C}$. The original top cover is replaced by a custom-made lid with a small optical window at the center. A high speed camera (Photron SA6) is mounted above the optical window, and a round LED light is attached below the top lid to illuminate the droplet. A speaker (Tang Band W3-2108) sits inside of the freezer just below the optical window. The speaker is driven by a function generator (Frederiksen), and the frequency and amplitude of the speaker are calibrated by a laser vibrometer. A substrate made of 0.22- $m m$-thick silica glass (Hampton Research Corp., HR8-082) is attached to the top of the speaker. Substrates were washed with acetone, alcohol, distilled water, and dried with a clean, filtered, low-humidity air flow before the experiment. A flow of filtered dry air $\left(2 \mathrm{~L} \mathrm{~min}^{-1}\right)$ is fed into the freezer to decrease the inside relative humidity and to ensure no dew or frost can form on the substrate at temperatures above $-20{ }^{\circ} \mathrm{C}$. After three hours the freezer is in a steady state, and the temperature close to the droplet is $-17.0 \pm 0.5{ }^{\circ} \mathrm{C}$, which is measured by a RTD probe near the substrate.

A $30 \mu L$ droplet of type 1 grade water (distilled, deionized, UV-irradiated) rests on top of the substrate at the beginning. The droplet can survive as a supercooled 
liquid for several hours on the substrate until it totally evaporates, because the natural freezing temperatures of the $30 \mu L$ droplet on a silica glass substrate is $-25.6 \pm 0.6^{\circ} C$, far below the chamber temperature. The natural freezing temperature is measured using the approach described in prior work[41]. Briefly, the substrate is set on a cold stage with a $2 K$ cooling rate, and the stage temperature is recorded when the droplet freezes 'naturally', i.e., without any external agitation. For the oscillation experiments described in this work, the supercooled water drop is allowed to sit on the substrate for 10 minutes before the speaker is turned on, to make sure droplet reaches the equilibrium temperature. After 10 minutes, the speaker is turned on with a specified frequency and amplitude, and it is observed whether the drop will freeze or not within 10 seconds. An inclined mirror is placed at the edge of the speaker, and the overhead camera then can also be used to record a side view when needed. The contact angle of silica glass is $93.9 \pm 4.4^{\circ}$, and the advancing and receding contact angles are $101.0 \pm 7.7^{\circ}$ and $79.0 \pm 3.9^{\circ}$, respectively. Advancing/receding contact angles are measured with a KRUSS G10 drop shape analyzer. The difference between advancing and receding contact angle is a measure of the pinning that occurs on silica glass substrates. 


\section{Results and Discussion}

The response of sessile droplets on a vertically oscillating plane has been well studied[87, 88]. The resonant frequency of the sessile droplet mainly depends on the mass of the droplet and the contact angle. To find the resonant frequency, we record the response of the droplet from a side view by increasing the frequency in 5 $\mathrm{Hz}$ increments, at a small amplitude. The resonant frequency for the first mode of a $30 \mu L$ droplet on the silica glass substrate is approximately $55 \mathrm{~Hz}$. For the vibrational freezing experiment, we use $30 \mathrm{~Hz}$, because we want to be away from the resonant frequency in order to keep the oscillation of the droplet simple.

A sessile droplet on a constant-frequency, vertically-oscillating substrate experiences two types of oscillations[87]: (1) at small amplitude, the contact line remains pinned, resulting in contact angle hysteresis; (2) at large amplitude, the contact line can move. The relative spreading distance of a $30 \mu L$ pure water droplet on a silica glass substrate, for various amplitudes at a vibration frequency of $30 \mathrm{~Hz}$ are shown in Figure 3.1 A. Amplitude is represented by the maximum velocity $(v$ in unit of $\mathrm{cm} / \mathrm{s}$ ) of the speaker for each case, with $v=\omega A$. Equal-time increments from individual experiments are separated by vertical red dash lines. The thick blue line is the relative spreading distance measured from a side view. The thin blue line is the uncertainty in the estimation. The relative spreading distance is defined as $\left(D(t)-D_{0}\right) / D_{0}$, 


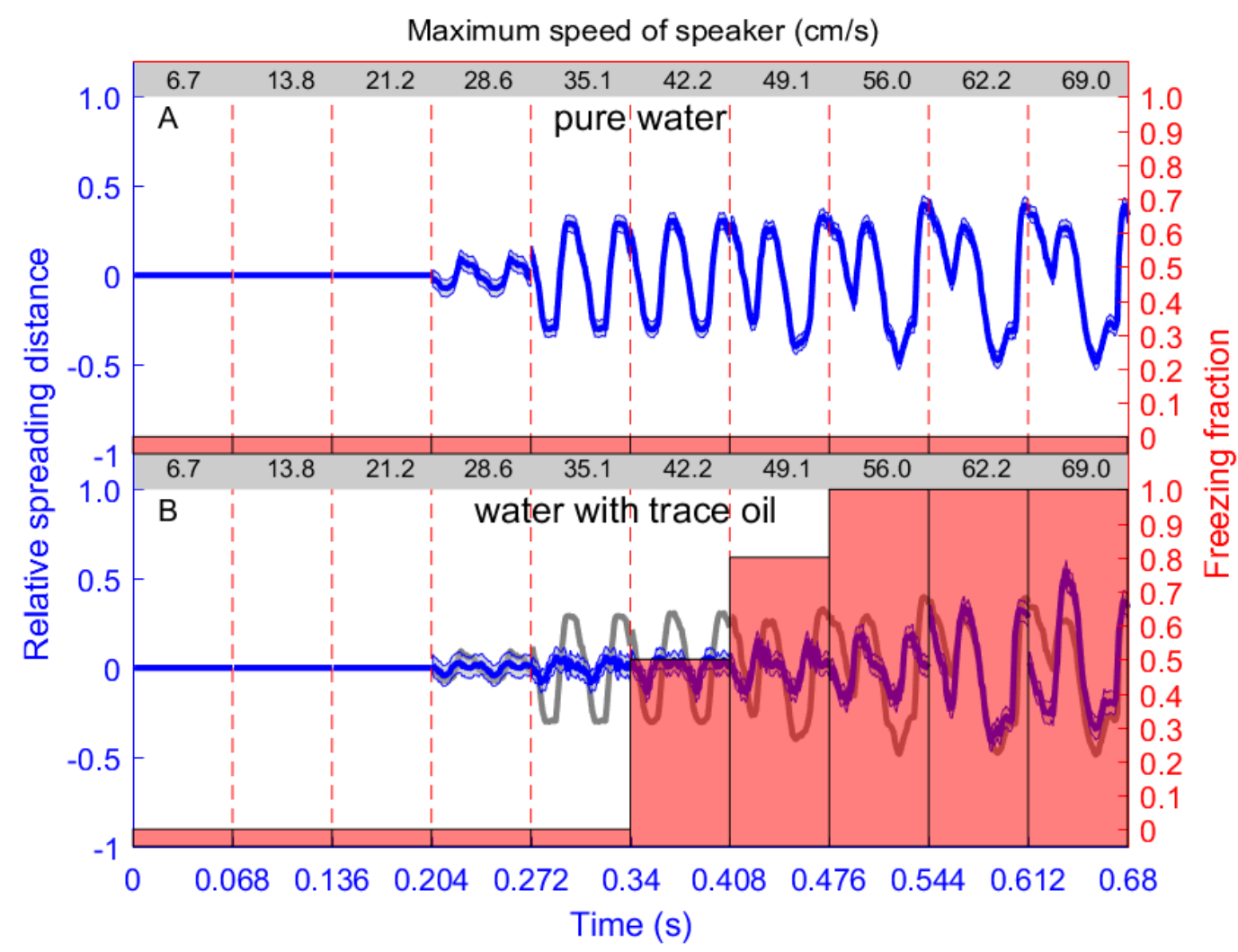

Figure 3.1: Response of a $30 \mu L \mathrm{~A}$ ) pure water and B) water with 10 $\mathrm{mg} / \mathrm{ml}$ pump oil on a silica glass substrate for different amplitudes at $30 \mathrm{~Hz}$ and $-17.0 \pm 0.5^{\circ} \mathrm{C}$.

where $D(t)$ is the diameter captured from the side view using $1000 \mathrm{~Hz}$ frame rate with $27.8 \mu m$ resolution, and $D_{0}$ is the diameter of droplet before vibration. It can be seen that the contact line does not move when $v_{\max }<28.6 \mathrm{~cm} / \mathrm{s}$ due to pinning on the substrate. The results also show that relative spreading of the drop is repeated within one oscillation cycle between $28.6 \mathrm{~cm} / \mathrm{s}$ and $42.2 \mathrm{~cm} / \mathrm{s}$, while it is repeated within two vibrational cycles between $49.1 \mathrm{~cm} / \mathrm{s}$ and $69.0 \mathrm{~cm} / \mathrm{s}$. This non-symmetric behavior at higher amplitude is because one satellite droplet becomes separated from the parent droplet vertically every other oscillation cycle. The smallest spreading 
distance is where the detachment occurs (see Figure $3.2 \mathrm{C}$ ). The detached satellite droplet can merge with the parent droplet, and a new cycle starts. When $v_{\max }>69.0$ $\mathrm{cm} / \mathrm{s}$, the droplet will quickly either shift out of the substrate, or breakup to several small satellite droplets within 10 seconds, which is not considered in this study.
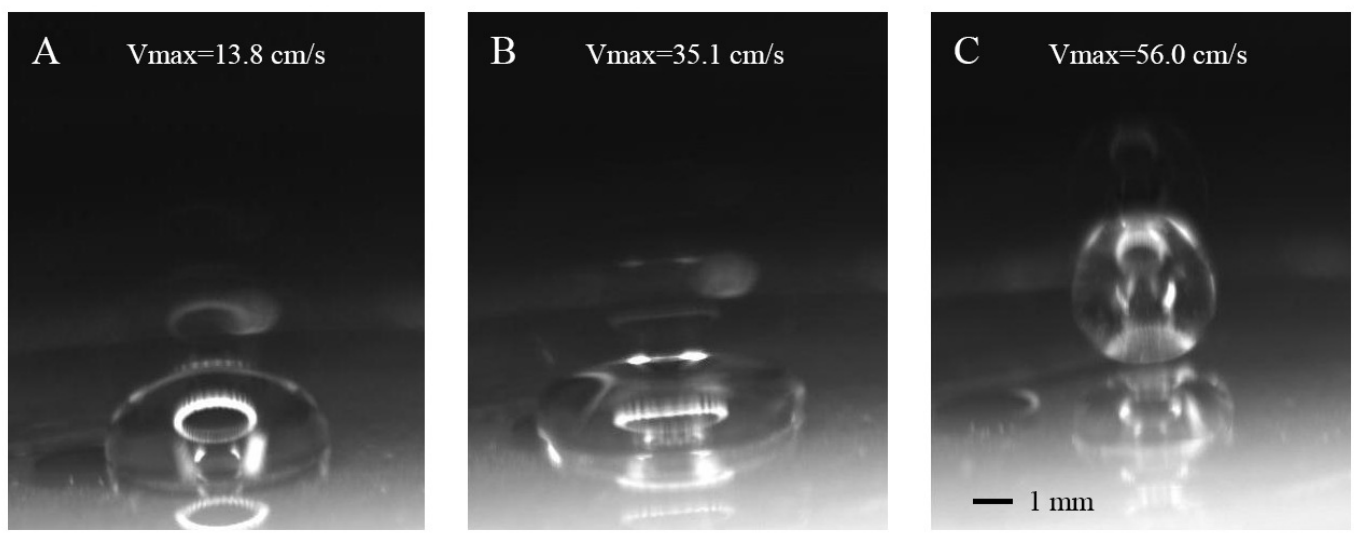

Figure 3.2: Side view of the response of a $30 \mu L$ drop changes with amplitude at three different amplitudes.

The freezing experiments are performed by setting a $30 \mu L$ drop of pure water at the center of the substrate, leaving it motionlessly in the cold chamber for 10 minutes before turning on the oscillation. The oscillating drop is observed for 10 seconds and occurrence of freezing is recorded. Experiments are repeated ten times for each case, and the freezing fraction is shown in Figure 3.1 A. The red bars represent the fraction of drops that experience freezing (freezing probability) for each case. It shows that ice nucleation is not triggered on silica glass substrate over the full range of amplitude. Apparently, although the existence of a moving contact contact line was observed to be necessary in prior experiments [152], it is not sufficient for initiating nucleation of 
ice in supercooled water.

The observation changes dramatically, however, when we mix a trace amount of oil with the water. Blue lines in Figure $3.1 \mathrm{~B}$ shows the result for a $10 \mathrm{mg} / \mathrm{mL}$ mixture (approximately $1 \%$ by mass of pump oil in water). Grey lines are the response of pure water for comparison. It can be seen that oil significantly inhibits the movement of the droplet due to its large viscosity. The addition of oil does not change the natural freezing temperature of the water drop. This result is not surprising because oil and water segregate. For example, prior studies demonstrate that oil will concentrate around the water droplet, concentrating at the three phase contact line[125]. However, things are quite different when contact line motion occurs: freezing is triggered when de-pinned oscillations are initiated, with the freezing probability increasing with amplitude (Figure 3.1 B).

Examples of time-resolved images of the freezing process taken with the high speed camera (5000 fps) are displayed in Figure 3.3. A1 and B1 represent the state before oscillation. For water, A2, A4, and A6 are examples of maximum spreading area, while A3 and A5 are examples of minimum spreading area. For the water-oil mixture, B2 is an example of maximum spreading area and B3 is an example of minimum spreading area before freezing. B4, B5 and B6 show how ice nucleates at the edge and how it propagates inward. Yellow arrows point out the multiple ice nucleation sites. For pure water, the drop oscillates with a de-pinned contact line but no freezing 
occurs. For the water-oil mixture, the droplet oscillates before freezing, but the maximum spreading area is smaller than pure water. Observation of the spatial location of the freezing onset shows that it always starts from the drop edge, near the contact line, and that sometimes it can even start from multiple points around the edge, as shown in Figure 3.3B4. This phenomenon is very similar to the electrowetting experiment: freezing from the edge and from multiple points[152]. We therefore anticipate that the mechanism of ice nucleation in the two cases should be similar.

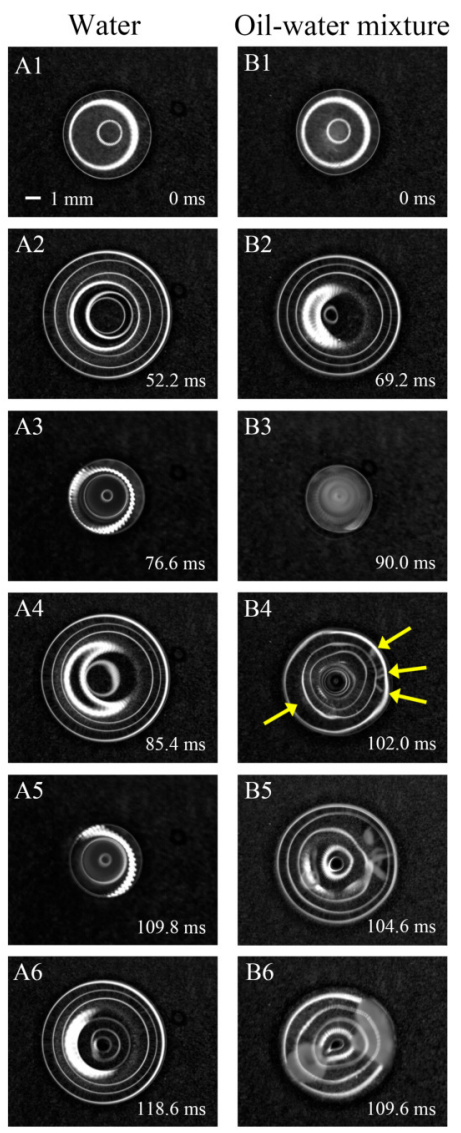

Figure 3.3: Individual video frames showing water (first column) and water with $10 \mathrm{mg} / \mathrm{mL}$ pump oil (second column) at different stages of oscillation on silica glass substrate with $30 \mathrm{~Hz}$ and $v_{\max }=56.0 \mathrm{~cm} / \mathrm{s}$ at $-17.0 \pm 0.5$ ${ }^{\circ} \mathrm{C}$. 
In order to investigate whether the ice nucleation effect observed in the water containing a trace of pump oil is due to unique chemical properties, we also test mineral oil. Results show that there is no significant difference between pump oil and mineral oil (compare Figure 3.1 and 3.4). We can also trigger ice nucleation of supercooled water droplets with trace amount of mineral oil through oscillation. High speed video confirms that ice nucleation always starts near the moving contact line, and can occur at multiple points. One example is shown in Figure 3.5.

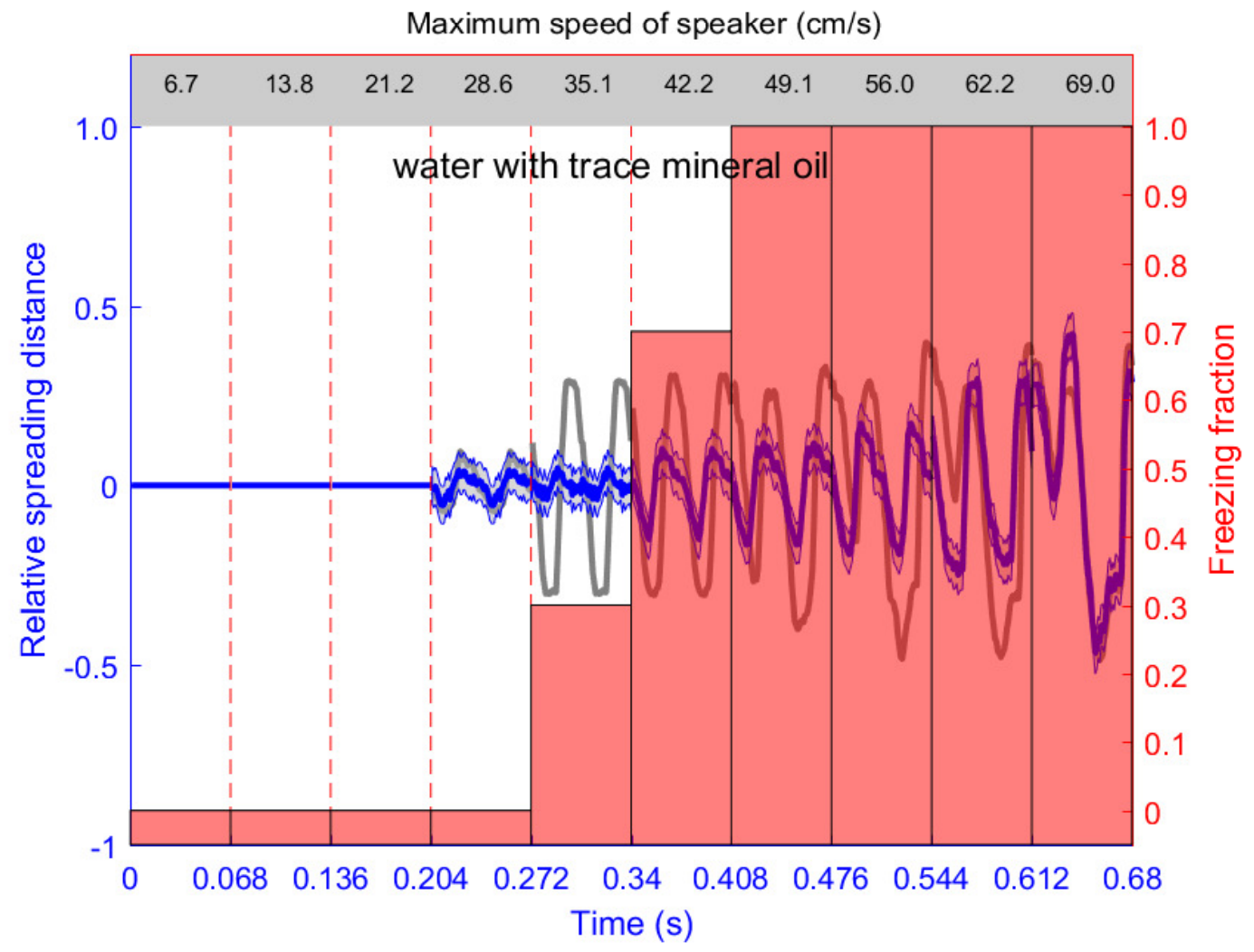

Figure 3.4: Response of a $30 \mu L$ water with $10 \mathrm{mg} / \mathrm{ml}$ mineral oil on a silica glass substrate for different amplitudes at $30 \mathrm{~Hz}$ and $-17.0 \pm 0.5^{\circ} \mathrm{C}$.

Seven different concentrations (ranging from $10^{-5}$ to $10 \mathrm{mg} / \mathrm{mL}$ ) of both pump oil 

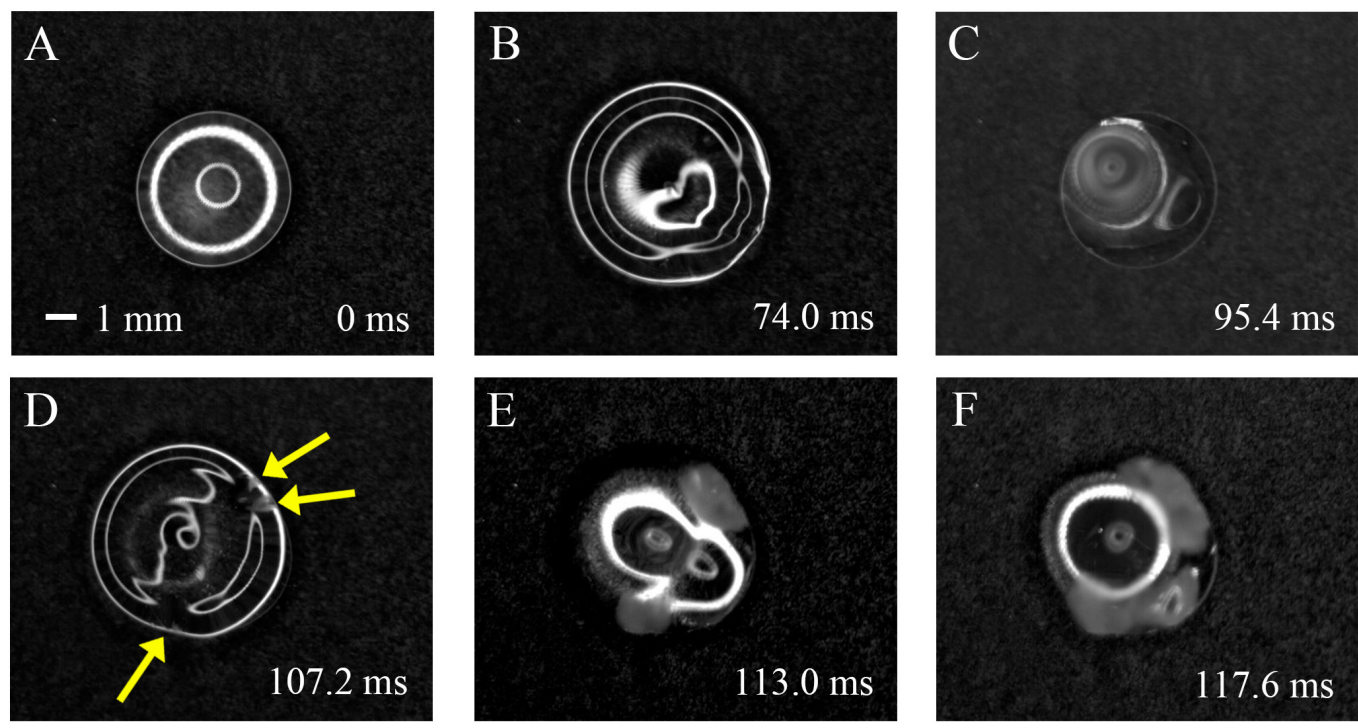

Figure 3.5: Individual video frames showing water with $10 \mathrm{mg} / \mathrm{mL}$ mineral oil at different stages of oscillation on silica glass substrate with $30 \mathrm{~Hz}$ and $v_{\max }=56.0 \mathrm{~cm} / \mathrm{s}$ at $-17.0 \pm 0.5^{\circ} \mathrm{C}$.

and mineral oil are also tested to study the effect of oil concentration on ice nucleation through oscillation. Results show that when the oil concentration is low, the freezing probability is low (see blue lines [3.6). The lower limit can be considered an oil concentration of 0 , i.e., pure water, for which the freezing fraction is 0 . The freezing fraction saturates at probability 1 for high concentrations. However, if we surround the pure water droplet with oil (much higher mixing fraction than $10 \mathrm{mg} / \mathrm{mL}$ ), then effectively the droplet becomes immobile, and we cannot trigger freezing even at very high amplitude and frequency. So our experiments suggest that in order to trigger ice nucleation on silica glass substrate upon oscillation, a trace of oil is needed, but too much oil alters the behavior. Results also show that the mean time for onset of freezing after starting the oscillation decreases with increasing oil concentration (see 
red lines in Figure (3.6). At the highest concentration shown in the figure, the freezing process is sufficiently rapid that it appears by eye to be instantaneous.

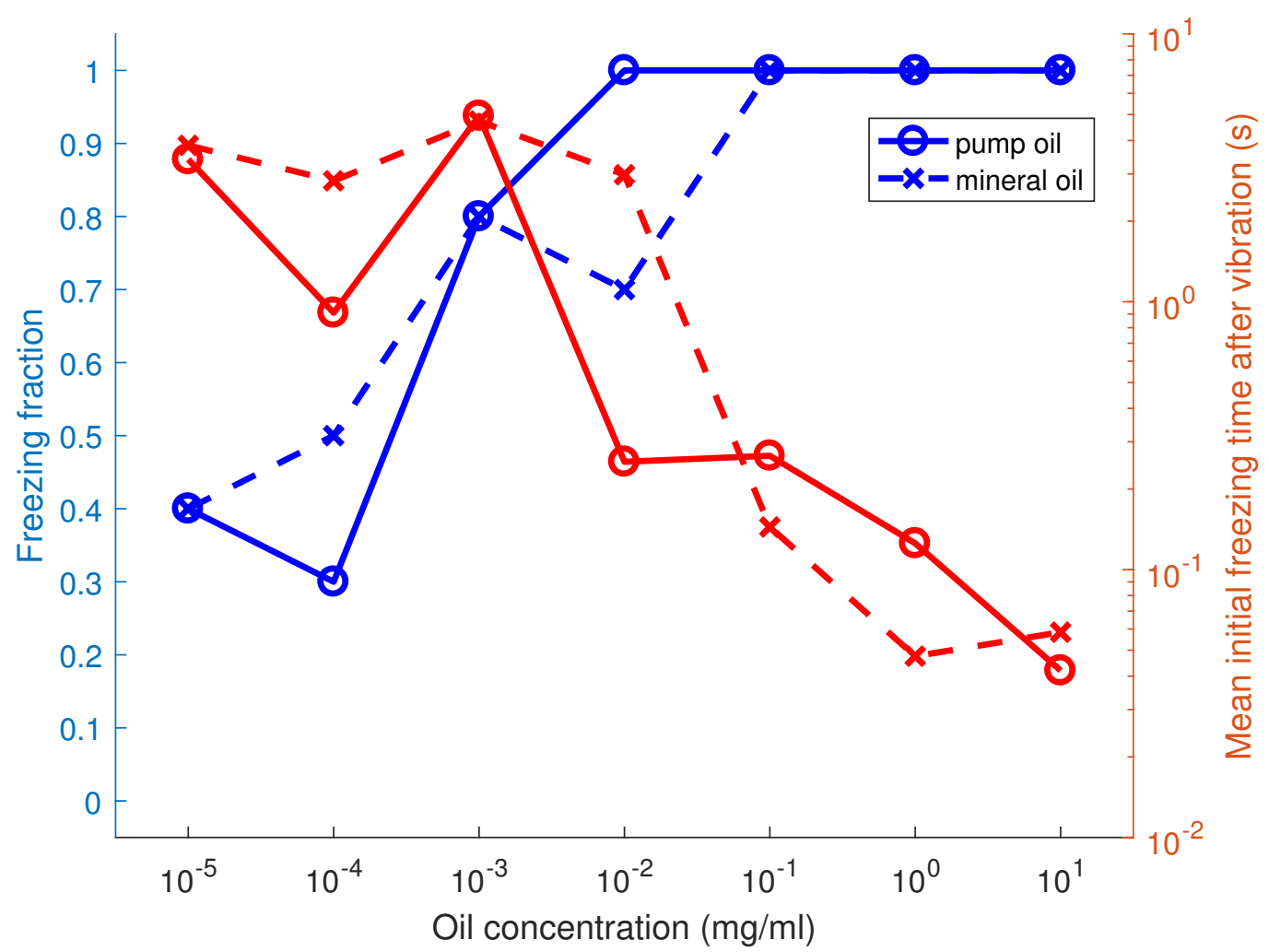

Figure 3.6: Effect of oil concentration on freezing fraction (blue lines) and freezing delay time after start of oscillation (red lines) with vibration of 30 $\mathrm{Hz}$ and $v_{\max }=56.0 \mathrm{~cm} / \mathrm{s}$ at $-17.0 \pm 0.5{ }^{\circ} \mathrm{C}$. Each case is repeated ten times.

So what's the ice nucleation mechanism? Because the oscillating drop experiments are performed at the same temperature $\left(0.5^{\circ} \mathrm{C}\right.$ uncertainty $)$, much higher than the natural freezing temperature, a temperature effect on ice nucleation can be ruled out. Furthermore, it is unlikely due to a chemical property of oil, because the oil is expected to be at the surface of the water droplet, and our measurements show 
that presence of the oil does not change the natural freezing temperature in a static drop. The natural freezing temperatures for $10 \mathrm{mg} / \mathrm{mL}$ pump oil and mineral oil are $-25.8 \pm 0.6{ }^{\circ} \mathrm{C}$ and $-26.5 \pm 0.4{ }^{\circ} \mathrm{C}$, similar to pure water droplet $-25.6 \pm 0.6{ }^{\circ} \mathrm{C}$. A possible role of frost or other surface defect is ruled out because a pure water drop experiences no freezing on the same substrate, and because no freezing occurs in the water-oil drop at small oscillation amplitude even when the contact line is moving back and forth. An explanation depending on a special site is also difficult to reconcile with the observation of freezing simultaneously initiated at multiple points because, usually, in such cases nucleation at one point is followed by nearly instant freezing of the full drop[40].
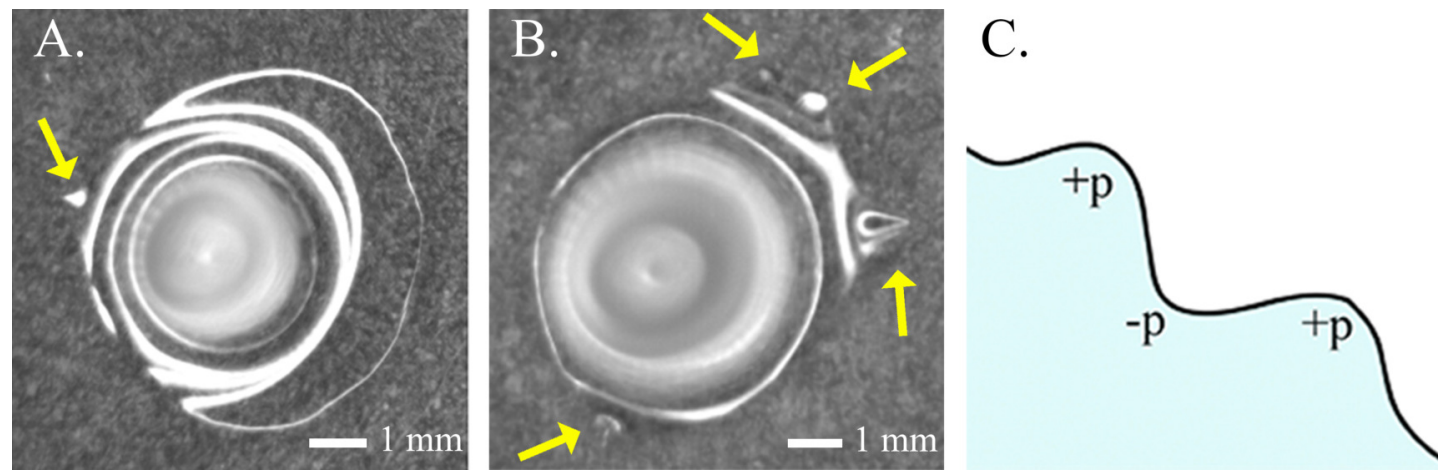

Figure 3.7: Example of macroscopic pinning behavior during oscillation of A) the water-oil mixture on a silica glass substrate and B) pure water on a PDMS substrate. C) Sketch of a curved contact line with indication of local pressure perturbations (+ or -). The scale is arbitrary, but can be expected to extend to nanometers, where the equivalent temperature change is of order degrees $\mathrm{K}$.

Whatever the mechanism, it must be related to drop oscillation and moving contact line, rather than temperature, chemistry of oil, or defect on substrate. The starkly 
different freezing behavior of the water-oil mixture compared with pure water must be the result of different contact line response during oscillation. The high speed camera images indeed show a difference: the shape of the pure water drop remains symmetric (spherical-cap shape) during the oscillations, whereas the shape of the water-oil droplet does not. Instead, the contact line often becomes strongly distorted from its static, circular shape, as shown in Figure 3.7 A. The contact line distortion during oscillation is likely a result of non-uniform distribution of oil at the surface due to the Plateau-Rayleigh instability [22]. Previous observation do show that oil is not uniformly distributed around the droplet[125]. The portions of the contact line containing oil will move slower than those regions without oil, due to the high viscosity of oil. The differential velocity leads to local distortions of the contact line, and we can therefore hypothesize that the existence of contact line distortion is associated with non-thermal initiation of ice nucleation.

We evaluate this hypothesis by asking whether we can induce strong distortion or curvature of the contact line in some other way. One possibility is to investigate drop oscillation on a substrate with localized pinning properties. It is known, for example, that physical (e.g., irregularities in surface morphology) or chemical (e.g., stains or inhomogeneities) defects on a surface can lead to strong pinning of the contact line[22]. As observed in Fig. 1 already, pinning also exist on the silica glass substrate, as manifested by the contact angle hysteresis. But the pinning on silica glass is quite uniform and homogeneous, which is why the shape of the droplet remains 
spherical during oscillation. We can ask, however, whether inhomogeneous, localized pinning can generate curved contact line and associated enhancement of freezing. A thin layer of PDMS spin-coated on a silica glass substrate is used in this study to test the hypothesis.

The PDMS (polydimethylsiloxane) surface fabrication is accomplished through spin coating. In brief, room temperature vulcanizing PDMS (Dow Corning RTV-3140) was dissolved in toluene and prepared in a 1:5 (w/v) PDMS solution. Siliconized glass coverslips were coated with PDMS using a two-stage spin coating process (Chemat Scientific KW-4A) to coat the siliconized glass coverslip. In the first stage, the coverslip underwent $1000 \mathrm{rpm}$ for $10 \mathrm{~s}$. At the beginning of Stage 1, $500 \mu L$ of PDMS solution was pipetted to the center of the spinning coverslip. Then the spin rate was increased to $6000 \mathrm{rpm}$ for another $40 \mathrm{~s}$ in Stage 2 for removing the excessive polymer. After spin coating, the PDMS-coated coverslip was left in a chemical hood at room temperature for further air-drying and curing for 20 hours. The thickness of the PDMS layer is about $5 \mu \mathrm{m}$. This value is estimated, according to the total mass applied to onto the coverslip, surface area of the coverslip, and the density of RTV3140. The contact angle of water on the PDMS substrate is $107.8 \pm 1.9^{\circ}$, and the advancing and receding contact angles are $113.5 \pm 5.4^{\circ}$ and $89.7 \pm 5.4^{\circ}$, respectively. The natural freezing temperature for a static water drop on PDMS is $-24.2 \pm 0.4{ }^{\circ} \mathrm{C}$.

The high speed camera confirms that the shape of a pure water droplet on a PDMS 
substrate, during oscillation with contact line motion, is not symmetric due to locally strong pinning (see Figure 3.7 B). Consistently, the contact line movement is also suppressed on the PDMS substrate compared with the silica glass substrate (Figure $3.8 \mathrm{~A})$. The contact line starts to move when $v_{\max } \geq 42.2 \mathrm{~cm} / \mathrm{s}$, substantially larger than that observed for the silica glass substrate. Furthermore, oscillating drops on PDMS at $-17^{\circ} \mathrm{C}$ are observed to freeze only after the contact line begins to move (Figure $3.8 \mathrm{~B}$ ) and again, the nucleation sites are all near the contact line. Water on strongly-pinning PDMS therefore behaves analogously as water with a trace of oil on silica glass.

All of the observations made thus far are consistent with the role of a distorted or strongly curved contact line. How can a curved contact line be connected to ice nucleation, a process notable for its strong temperature dependence? We take as our motivation the notion of nucleation as a kinetic phenomenon involving a Gibbs free energy barrier[51], and the fact that the natural variables for Gibbs free energy are temperature and pressure. Local curvature of a droplet surface or contact line is associated with a perturbation Laplace pressure[22] (see Figure 3.7 C). Recent work directly shows that the Laplace pressure affects the chemical potential difference between ice and water that drives phase change, thus modifying the ice nucleation rate: For example, the Laplace pressure of a nano-droplet is positive and non-negligible, explaining why a nanoscale supercooled droplet can survive at very low temperature without freezing [69]. It is therefore reasonable to expect that a negative pressure 

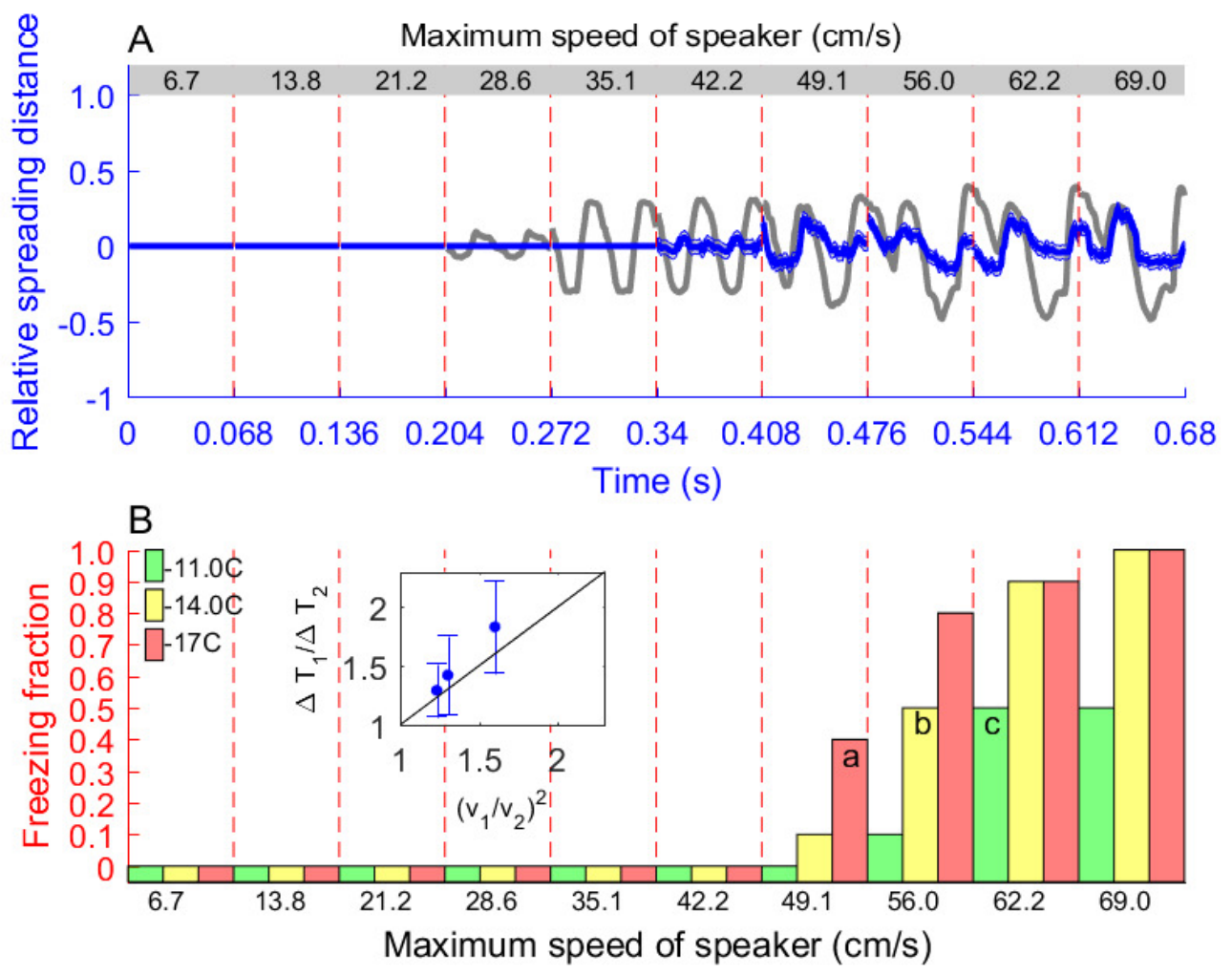

Figure 3.8: A) Response of a $30 \mu L$ pure water droplet on a PDMS substrate for different amplitudes at $30 \mathrm{~Hz}$. Format and line styles are as in Figure 3.1, B) Fraction of droplets that freeze for different amplitudes with $30 \mathrm{~Hz}$ at three temperatures.

perturbation associated with locally-negative curvature will enhance the local ice nucleation rate. This would be consistent with the observation that ice can form at anomalously high temperatures in the presence of deeply negative pressure in a liquid capillary bridge[90].

Previous computational and theoretical studies show that pressure will affect the 
chemical potential difference between ice and water for the phase change as [69, 82]

$$
\Delta \mu=l_{f} \frac{\Delta T}{T_{0}}+\Delta p \Delta \nu
$$

where $\Delta p$ can be either a positive or negative pressure perturbation and $\Delta \nu=\nu_{l}-\nu_{s}$ is the difference in specific volumes for liquid and solid water. Because $\Delta \nu$ is negative for the water-ice system (i.e., water density anomaly), the sign of $\Delta p$ determines whether pressure will increase or decrease the driving force for a phase change $\Delta \mu$, thus enhancing or suppressing the ice nucleation rate $J$. For example, the Laplace pressure of a nano-droplet is positive and may explain why nanoscale supercooled droplet can survive at very low temperature without phase change[69]. Conversely, it has also been observed that deeply negative pressure in a liquid capillary bridge allows ice to form at high temperatures[90].

Because negative pressure can increase the chemical potential difference between supercooled liquid and ice, we will consider the role of pressure perturbations in the heterogeneous ice nucleation rate. That, in turn, will allow for estimation of the negative pressure required to have the same ice nucleation rate at a higher temperature. The ratio of the heterogeneous ice nucleation rate at a higher temperature $T^{\prime}$ to that at a lower temperature $T$ at $p_{0}$ is $[64]$

$$
\frac{J\left(p_{0}, T^{\prime}\right)}{J\left(p_{0}, T\right)}=\exp \left[-\frac{16 \pi \sigma_{l s}^{3} f_{\text {het }}}{3 k_{B} \rho^{2} l_{f}^{2}}\left(\frac{T_{0}^{2}}{T^{\prime} \Delta T^{\prime 2}}-\frac{T_{0}^{2}}{T \Delta T^{2}}\right)\right]
$$


where $k_{B}$ is Boltzmann constant, $\sigma_{l s}$ is the water-ice surface free energy, $\rho$ is the density of ice, $l_{f}$ is the enthalpy of fusion, and $f_{\text {het }}$ is a geometrical factor accounting for the heterogeneous nucleation efficiency of a substrate. Here we assume the prefactor does not change significantly with temperature. This is roughly true when $T^{\prime}$ is close to $T$, compared to the exponential term that is retained. Using Eq. 3.1, the ratio of heterogeneous ice nucleation rate due to pressure perturbation $\Delta p$ at the same temperature $T^{\prime}$ is

$$
\frac{J\left(p_{0}+\Delta p, T^{\prime}\right)}{J\left(p_{0}, T^{\prime}\right)}=\exp \left[-\frac{16 \pi \sigma_{l s}^{3} f_{h e t}}{3 k_{B} \rho^{2} l_{f}^{2}}\left(\frac{T_{0}^{2}}{T^{\prime}\left(\Delta T^{\prime}+T_{0} \Delta p \Delta \nu / l_{f}\right)^{2}}-\frac{T_{0}^{2}}{T^{\prime} \Delta T^{\prime 2}}\right)\right] .
$$

When the enhancement due to pressure perturbation equals the suppression due to temperature, $J\left(p_{0}+\Delta p, T^{\prime}\right)=J\left(p_{0}, T\right)$, which leads to

$$
\Delta p \Delta \nu=\frac{l_{f}}{T_{0}} \sqrt{\frac{T}{T^{\prime}}} \Delta T-\frac{l_{f}}{T_{0}} \Delta T^{\prime}
$$

If $T^{\prime}$ is close to $T$, this can be approximated as

$$
\Delta p=\frac{l_{f}}{T_{0} \Delta \nu}\left(T^{\prime}-T\right)
$$

It tells us negative pressure we need to have the same ice nucleation rate at higher temperature is proportional to the temperature difference. For example, in order to balance the suppression of ice nucleation rate resulting from a $\sim 10 \mathrm{~K}$ temperature 
increase, a negative pressure of $\sim 10^{8} \mathrm{~Pa}$ is required, which assuming static Laplace pressure corresponds to a radius of curvature on the order of $1 \mathrm{~nm}$. These values are plausibly achieved, given observations of surface roughness, pinning deformation, and cavity collapse [50, 56, 93].

Our optical system does not possess the resolution necessary to measure curvature below the micrometer-scale, but we do observe a clear correlation between macroscopic curvature and the ability to trigger ice nucleation by drop oscillation, regardless of whether the distorted contact line is due to the pinning or Plateau-Rayleigh instability for the water-oil system, or for inhomogeneous pinning of the PDMS substrate (see Figure 3.7). The curved contact line will generate local perturbations in Laplace pressure around the edge, and the regions of negative radius of curvature will lead to negative Laplace pressure and enhancement of the ice nucleation rate. Another way to generate negative pressure, which cannot be ruled out, is through cavity collapse[44, 50]. Although we have no direct evidence supporting it, it is plausible that the moving, distorted contact lines lead to cavity formation through relatively small pressure perturbations. The collapse of the cavity then produce very high positive, and then negative pressure, which can lower the freezing temperature of supercooled water [49, 51, 108]. Regardless of the details, the observations of enhanced freezing are consistent with a predominant role of negative pressure.

The notion of pressure-induced nucleation implies that ice formation is favored when 
water is either supercooled or de-stressed. Can the correspondence between temperature and negative pressure (or other off-diagonal terms of the stress tensor [8, 61] ) be demonstrated experimentally? To that end, Figure 3.8 B also contains freezing results for $-11^{\circ} \mathrm{C}$ and $-14{ }^{\circ} \mathrm{C}$, showing that the freezing probability at a higher temperature is nearly compensated by more intense agitation (higher oscillation amplitude). For example, a freezing probability near 0.5 is observed for $-17^{\circ} \mathrm{C}$ when there is a relatively low amplitude of $49.1 \mathrm{~cm} / \mathrm{s}$; then for $-14{ }^{\circ} \mathrm{C}$ requires $56.0 \mathrm{~cm} / \mathrm{s}$, and finally at $-11^{\circ} \mathrm{C}$ a relatively high amplitude of $62.2 \mathrm{~cm} / \mathrm{s}$ is required. To test the compensation condition quantitatively, we note that Eq. 3.5 yields $(\Delta T)_{1} /(\Delta T)_{2}=(\Delta p)_{1} /(\Delta p)_{2}$, where 1 and 2 denote the experimental conditions at different $(p, T)$ but with the same ice nucleation rate. The green, yellow, and red bars represent the freezing fraction for each case at $-11.0 \pm 0.5{ }^{\circ} \mathrm{C},-14.0 \pm 0.5$ ${ }^{\circ} C$, and $-17.0 \pm 0.5{ }^{\circ} C$, respectively. Associating Bernoulli-type scaling $\sim \rho v^{2}$ with $\Delta p$, we see in the inset of Fig. $4 \mathrm{~B}$ that, indeed, the equality is supported to within the experimental uncertainty: with $\Delta p_{c} / \Delta p_{b} \sim\left(v_{c} / v_{b}\right)^{2} \sim(62.2 / 56.0)^{2}=1.23$ compared to $\Delta T_{c} / \Delta T_{b}=(24.2-11.0) /(24.2-14.0)=1.29, \Delta p_{b} / \Delta p_{a} \sim 1.30$ compared to $\Delta T_{b} / \Delta T_{a}=1.42$, and $\Delta p_{c} / \Delta p_{a} \sim 1.60$ compared to $\Delta T_{c} / \Delta T_{a}=1.83$ Here a, b, and $\mathrm{c}$ are labeled in the red, yellow, and green bars with near 0.5 freezing probability in Figure 3.8 B. Note that $\Delta T_{1} / \Delta T_{2}$ is closer to $\left(v_{1} / v_{2}\right)^{2}$, as opposed to $v_{1} / v_{2}$ or $\left(v_{1} / v_{2}\right)^{3}$. Uncertainties shown represent the observed temperature variability.

The oscillating drop experiments described here confirm that non-thermal distortion 
of the contact line strongly enhances the freezing of supercooled water. The notion of pressure-induced ice nucleation provides compelling context for interpretation of many prior experiments: Droplet freezing triggered by impaction of ice nuclei, salt particles, or another supercooled droplet, may all rely on the pressure perturbation through collision [34, 50, 85]. In addition, the enhanced ice nucleation at the contact line on particles or nanotextured surfaces may also be a result of the local curved contact line due to strong inhomogeneous pinning[26, 39, 56]. Pressure-induced ice nucleation provides a physically based understanding that can be quantified for prediction of ice formation processes in a variety of applications, such as atmospheric ice nucleation. The observations and implications discussed in this paper set the stage for further investigation of pressure perturbations occurring during collisions between droplets and a substrate or particle, their quantitative enhancement of nucleation rate, and their implications for the phenomenon of contact nucleation.

\section{Acknowledgement}

This work was supported by DOE Office of Science as part of the Atmospheric System Research program through Grant No. DE-SC0011690. A. Kostinski acknowledges support from NSF AGS-1639868. We thank Dr. A. Barnard for assistance with the laser vibrometer measurements and Dr. W. Cantrell for helpful discussions. We sincerely thank Yujin Sun and Dr. Jaroslaw W. Drelich in the Department of MSE, 
MTU for assisting surface characterization and Dr. Megan C. Frost in the Department of BME, MTU for providing with different polymer substrates. 


\section{Chapter 4}

\section{Minimalist Model of Ice}

\section{Microphysics in Mixed-Phase}

\section{Stratiform Clouds}

This chapter details the minimalist model for mixed-phase stratiform clouds based on stochastic ice nucleation! 1 . This work was based on a research collaboration and is published in full form in the Geophysical Research Letters [148], and is reprinted with permission by the American Geophysical Union2.

\footnotetext{
${ }^{1}$ This paper is co-authored by M. Ovchinnikov and R.A. Shaw

${ }^{2}$ Reprinted with permision from: F. Yang, M. Ovchinnikov and R.A. Shaw, Geophysical Research Letters, 40, 3756, 2013. Copyright 2013 by Geophysical Research Letters.
} 


\section{Abstract}

The question of whether persistent ice crystal precipitation from supercooled layer clouds can be explained by time-dependent, stochastic ice nucleation is explored using an approximate, analytical model, and a large-eddy simulation (LES) cloud model. The updraft velocity in the cloud defines an accumulation zone, where small ice particles cannot fall out until they are large enough, which will increase the residence time of ice particles in the cloud. Ice particles reach a quasi-steady state between growth by vapor deposition and fall speed at cloud base. The analytical model predicts that ice water content $\left(w_{i}\right)$ has a 2.5 power law relationship with ice number concentration $n_{i} . w_{i}$ and $n_{i}$ from a LES cloud model with stochastic ice nucleation confirm the 2.5 power law relationship and initial indications of the scaling law are observed in data from ISDAC. The prefactor of the power law is proportional to the ice nucleation rate, and therefore provides a quantitative link to observations of ice microphysical properties.

\section{Introduction}

Long-lived mixed-phase clouds are frequently observed in the Arctic region [76, 117,

132] where they play an important role in the radiation balance [73]. Mixed-phase 
layer clouds with similar characteristics exist over many other regions of the earth as well [137]. Observations show that ice particles precipitate from these clouds nearly all the time [76]. Recent experiments and modeling studies have led to great strides in understanding the complex and coupled radiation, dynamics, and microphysics of these clouds [78], but why the mixed-phase clouds can exist for such a long time with steadily precipitating ice particles remains uncertain. One of the essential questions is how to replenish the ice nuclei that are quickly removed after activation and subsequent growth and sedimentation of ice crystals. It has been suggested that ice nuclei entrained from the top of the boundary layer might offset the ice particles lost at the cloud base $[3]$, and some tentative observational evidence for that has been found [52]. However, [33] concluded that ice nuclei concentrations above cloud top were too low to account for observed ice number concentrations in cloud, given realistic entrainment rates. [80] suggested it might be the existence of low efficiency contact ice nucleation that extends the mixed-phase cloud lifetime, while [32] showed that evaporation ice nucleation at cloud top may also help explain the persistence of mixed-phase clouds. Recently, [137] argued that a time-dependent ice nucleation process in supercooled layer clouds would be a plausible explanation for the observed persistence of ice precipitation, and that concept can be taken as one of the motivations for this work.

This chapter provides an analytical model describing ice microphysical properties in a mixed-phase stratiform cloud, with emphasis on understanding the balanced state of ice nucleation, vapor growth, and sedimentation. The model is referred to 
as 'minimalist' because we seek for the minimum number of physical assumption necessary to give a reasonable explanation for the presence of ice persisting over long time and also providing a reasonable estimate of the ice crystal mass and precipitation rate.

\section{Model Description}

The model is based on the assumption of horizontally uniform and steady state conditions, in which the rate of formation of ice crystals within the cloud is balanced by the rate of removal of ice crystals through precipitation. For simplicity, we assume the cloud has uniform temperature and possesses a sufficient quantity of supercooled water so that the cloud humidity can be taken as saturated with respect to liquid water throughout. Ice crystals are assumed to nucleate at a uniform rate in the cloud and then to grow by water vapor deposition as they settle through the cloud. The cloud top is assumed to be closed. Steady state implies that the humidity within the cloud, the cloud droplet concentration, and the ice particle concentration do not change with time.

To provide a context for this work, we draw on typical cloud properties observed for many hours on 26 April 2008 during the Indirect and Semi-Direct Aerosol Campaign $(I S D A C)$ [76, 92]. We set the cloud temperature at $-10^{\circ} C$ and thickness of the 
mixed-phase cloud at $h=150 \mathrm{~m}$. The ice equivalent diameter reached $1 \mathrm{~mm}$ near the cloud base, but there was little signature of aggregation or riming due to the low liquid and ice water content. The liquid water profile is close to adiabatic.

A central assumption in this model is that ice crystals form stochastically from plentiful ice nuclei, as suggested by recent laboratory work [83, 136]. Details of how the nucleation rates are distributed are not considered here, effectively neglecting any highly efficient ice nuclei as transients not relevant to the steady state. Ice crystals are assumed to originate from supercooled cloud droplets, with some fraction $\phi$ of the droplets containing ice nuclei. If the liquid cloud droplet concentration $n_{w}$ is homogeneous in the cloud, the number concentration of newly formed ice crystal number concentration in time $\Delta t$ can be written as $\Delta n_{i}=n_{w} \phi\left(1-e^{-\Delta t / \tau}\right)$, where $\tau$ is the characteristic time for heterogeneous ice nucleation (i.e., the inverse of the extensive nucleation rate). If $\tau>>\Delta t, \Delta n_{i} \approx n_{w} \phi \Delta t / \tau$, so we can define a volume ice formation rate $n_{i}^{\prime}=\lim _{\Delta t \rightarrow 0} \Delta n_{i} / \Delta t=n_{w} \phi / \tau$, with units of $m^{-3} s^{-1}$.

Once nucleated, ice crystals grow and settle. The ice crystal radius $r_{i}$ increases with time due to vapor deposition growth at the rate approximated as $r_{i} \frac{d r_{i}}{d t}=C D s_{i}[64$, where $C$ is a shape factor, $D$ is a modified diffusion coefficient accounting for heat transport and density, and $s_{i}$ is the supersaturation with respect to ice. At constant temperature in the environment saturated with respect to liquid water, $s_{i}$ is constant throughout the cloud, so if the initial radius of the crystal is ignored the result of 
time integration of the growth equation is $r_{i}^{2}=2 C D s_{i} t$.

The ice particle terminal speed $v_{i, t}$ is assumed to have a power-law relationship with ice particle radius as $v_{i, t}=b r_{i}^{k}$, where $b$ and $k$ may depend on ice crystal habit and mass. For large crystals experiencing turbulent drag we can safely take the exponent to be $k=1 / 2$. If the deposition growth equation for $r_{i}$ is substituted into the ice terminal speed equation and then integrated over the distance from the point of nucleation to cloud base, we obtain equivalent ice crystal diameter as a function of height. Even for crystals nucleated at the cloud top, however, the resulting sizes do not exceed $200 \mu m$ and cannot explain those observed in ISDAC. To obtain a realistic crystal size it is necessary to consider growth in the presence of updrafts.

Both observational data and model results show that updraft velocity in the cloud is maximum near the base and zero at the top of the cloud (see supplementary material). For simplicity, we assume a linear decrease of updraft velocity with altitude: $v_{e}(z)=$ $\frac{v_{0}(h-z)}{h}$, where $h$ is the cloud thickness and $z$ is altitude above cloud base. Thus the ice fall speed under the influence of background velocity is $v_{i}=-b\left(2 C D s_{i} t\right)^{\frac{k}{2}}+v_{e}(z)$. The result is a differential equation for the height of an ice crystal above the cloud base,

$$
\frac{d z}{d t}=-Q t^{k / 2}+P(h-z)
$$


where $P=v_{0} / h$ and $Q=b\left(2 C D s_{i}\right)^{k / 2}$. The first term tends to increase fall speed due to depositional growth, and the second is the opposing updraft. The differential equation has the solution $z=h-e^{-P t} \int Q t^{k / 2} e^{P t} d t-c e^{-P t}$ where $c$ is a parameter depending on the initial condition: If the ice particle forms at cloud base, $c=h$, whereas if the ice particle forms at cloud top, $c=0$.

Trajectories for growing crystals formed at cloud top and cloud base are shown in Figure 4.1. In order to compare with ISDAC observations we take $v_{0}=0.3 \mathrm{~m} \mathrm{~s}^{-1}$, $h=150 \mathrm{~m}$, and $v=17 r_{i}^{0.5}$, with $r$ in $\mathrm{m}$ and $v$ in $\mathrm{m} \mathrm{s}^{-1}$. Both particles stay in the cloud longer than $4000 s$ (see Figure 4.1(a)), allowing large crystal size to be reached. Figure 4.1(a) also shows that both trajectories merge so that ice crystals tend to congregate as they approach the cloud base. We refer to this as reaching a quasi-steady state at the lower region of the cloud: no matter where ice particle forms it will have the similar size in the cloud base region and the terminal speed of ice particles will be close to the background updraft speed. Quasi-steady state implies that the terms on the right side of Equation 4.1 are nearly balanced such that $\frac{d z}{d t} \approx 0$, and therefore

$$
z=h-\frac{Q}{P} t^{k / 2} .
$$

The solution from Equation 4.2 (black line in Figure 4.1) is slightly offset from the 

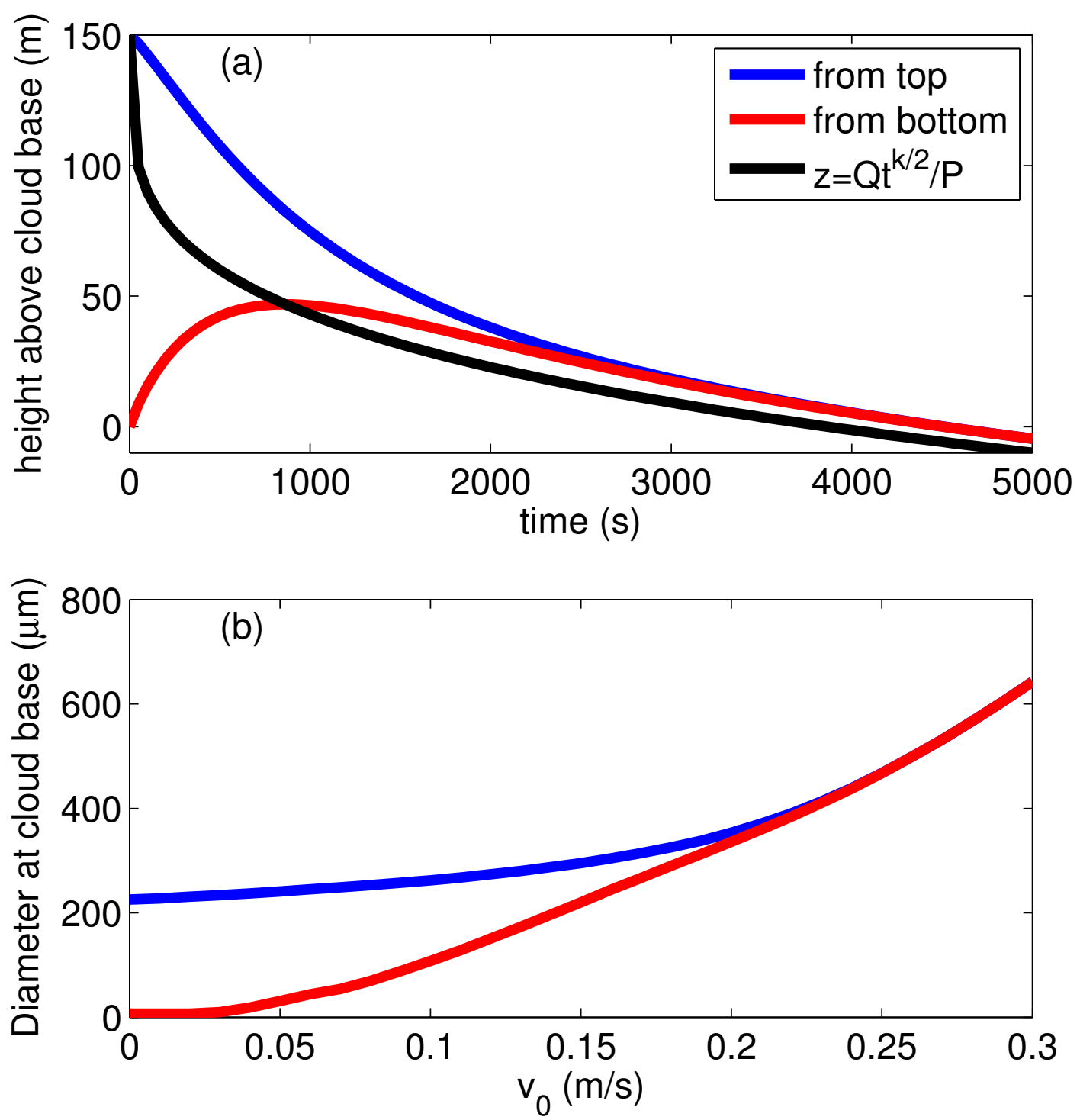

Figure 4.1: (a)Height of ice particle above cloud base vs time. Updraft velocity decreases linearly from $v_{0}=0.3 \mathrm{~m} / \mathrm{s}$ at the base to zero at the top. Blue line represents ice formed at cloud top, red at cloud base. Black line is based on quasi-steady state (Equation 4.2). (b) Diameter at cloud base of ice particles which forms at cloud top (blue) and cloud base (red) under different background updraft velocity $v_{0}$.

numerical solution of Equation 4.1 at the base region, but has very similar slope.

The slope of these curves represents the ice crystal fall speed, so the velocity at cloud base can easily be obtained by differentiating Equation 4.2, $v_{i}=(k Q / 2 P) t^{(k-2) / 2}=$ 
$(k / 2)\left(Q / v_{0}\right)^{2 / k} h$, where the second equality is obtained by eliminating $t$ using Equation 4.2, and taking $z=0$. At cloud base $v_{0}=b r_{i}^{k}$, so this becomes

$$
v_{i}=\frac{k h C D s_{i}}{r_{i}^{2}}=\frac{k h}{r_{i}} \frac{d r_{i}}{d t},
$$

where the second equality follows from the vapor growth rate equation. This result can be interpreted as the mean fall speed being proportional to the linear growth rate under quasi-steady state, i.e., the crystal only approaches cloud base at the rate at which it is able to grow by vapor deposition. Thus, in the quasi-steady regime the crystal growth times tend to converge to a single value, regardless of the initial location of the crystal nucleation event, and that time is much greater (and therefore the size much larger) than the time a growing crystal would take to fall through the depth of the cloud without an updraft.

It should be noted that Equation 4.3 is only satisfied in the quasi-steady region. To find when quasi-steady state can be expected at the cloud base region, different $v_{0}$ are tested (see Figure 4.1(b)). It can be seen that quasi-steady state is valid only when $v_{0}$ is larger than $0.2 \mathrm{~m} \mathrm{~s}^{-1}$. In addition, Figure 4.1(b) shows that if $v_{0}$ is larger than $0.25 \mathrm{~m} \mathrm{~s}^{-1}$, no matter where ice particles originate, they can reach diameters larger than $500 \mu \mathrm{m}$, which is close to the observed value in ISDAC. We take this as observational support for the highly simplified picture of quasi-steady ice crystal 
growth at cloud base. Physically it means that cloud regions containing relatively large updraft velocities (comparable to terminal velocity of large ice particles) will suspend small ice particles, increasing their residence time in the ice-supersaturated cloud.

Since in a quasi-steady state at cloud base all crystals have similar sizes and fall speeds (Figure 4.1) we can calculate the flux of ice particles out of the cloud as $n_{i} v_{i}$ and equate it with the column integrated nucleation rate

$$
n_{i} v_{i}=n_{i}^{\prime} h .
$$

Combining Equations 4.3 and 4.4, we get $n_{i}=n_{i}^{\prime} r_{i}^{2} / k C D s_{i}$. Using the definition of ice water content $w_{i}=n_{i} m_{i}=\frac{4}{3} \pi \rho_{i} r_{i}^{3} n_{i}$ to eliminate $r_{i}$, we obtain a relationship between $w_{i}$ and $n_{i}$,

$$
w_{i}=\frac{G^{\prime} n_{i}^{5 / 2}}{n_{i}^{\prime 3 / 2}},
$$

where $G^{\prime}=\frac{4}{3} \pi \rho_{i}\left(k C D s_{i}\right)^{3 / 2}$. This $5 / 2$ power law relationship between $w_{i}$ and $n_{i}$ is interesting because dilution or transport of ice crystals will tend to decrease $w_{i}$ and $n_{i}$ proportionally, i.e., they should follow a 1.0 power law. The prediction of 
a 2.5 power law is a result of ice particles being continuously formed due to the assumed stochastic ice nucleation process and smaller ice particles being held in the cloud by the updraft, with a concomitant increase in their residence time. This is consistent with the finding of [65] that a 1.0 power law exists between $w_{i}$ and $n_{i}$ for an exponential size distribution of snow crystals and no mean updraft.

\section{Comparison Of Results With LES Cloud Model And ISDAC Observations}

In order to evaluate the plausibility of the minimalist model we evaluate its predictions in the context of ISDAC cloud simulations and field observations. Two points should be mentioned as part of this comparison. First, the minimalist model is one dimensional, whereas the cloud simulations and observations contain all three dimensions. In the $1 \mathrm{D}$ model, ice particles can only fall out of the cloud in the one column, while in $3 \mathrm{D}$, ice particles can separate horizontally due to dilution or transport and can fall out of downdraft regions more quickly. So we expect that $G^{\prime}$ in Equation 4.5 can be modified to $G^{\prime}=\frac{4}{3} \gamma \pi \rho_{i}\left(k C D s_{i}\right)^{3 / 2}$, where $\gamma$ is a 3D correction parameter. Second, Equation 4.5 describes the $w_{i}-n_{i}$ relationship under the quasi-steady state conditions assumed in the model derivation, when updraft velocity exists in the cloud and linearly decreases with altitude. The extent to which the assumptions capture 
the essential physics is to be tested.

Large-Eddy Simulations (LES) are performed using the System of Atmospheric Modeling (SAM) dynamical framework [55] coupled with the Spectral Bin Microphysics (SBM) scheme [54] as described in [30]. The simulation setup for the ISDAC case is similar to that used by [92]. The model's computational domain includes $64 \times 64$ columns and 160 vertical levels, using $50 \mathrm{~m}$ grid spacing in both horizontal directions and $10 \mathrm{~m}$ in the vertical. Size distributions for liquid and ice hydrometeors are predicted, each discretely represented by 33 size bins. For ice particle properties, the same relationship between ice crystal size and fall speed as in the minimalist model are used. Collision processes are not considered. Specification of cloud condensation nuclei follows [92], producing a nearly constant droplet number concentration of around $200 \mathrm{~cm}^{-3}$ in the cloud. Ice particles are produced by prescribing a constant freezing probability of any droplet, regardless of its size, location, etc. Thus, a fraction $\left(n_{i}^{\prime} \Delta t\right.$, where $\Delta t$ is the model time step) of the droplet size distribution is converted to the ice size distribution every time step.

To test whether $w_{i}$ and $n_{i}$ from the LES exhibit a 2.5 power law similar to Equation 4.5, we analyze these variables from a time when the simulation has achieved a reasonably steady state (at 5 hours). We first select the columns containing an accumulation zone, where updraft velocity decreases with altitude in the cloud region, and then choose the corresponding $w_{i}$ and $n_{i}$ data at the base of the accumulation 

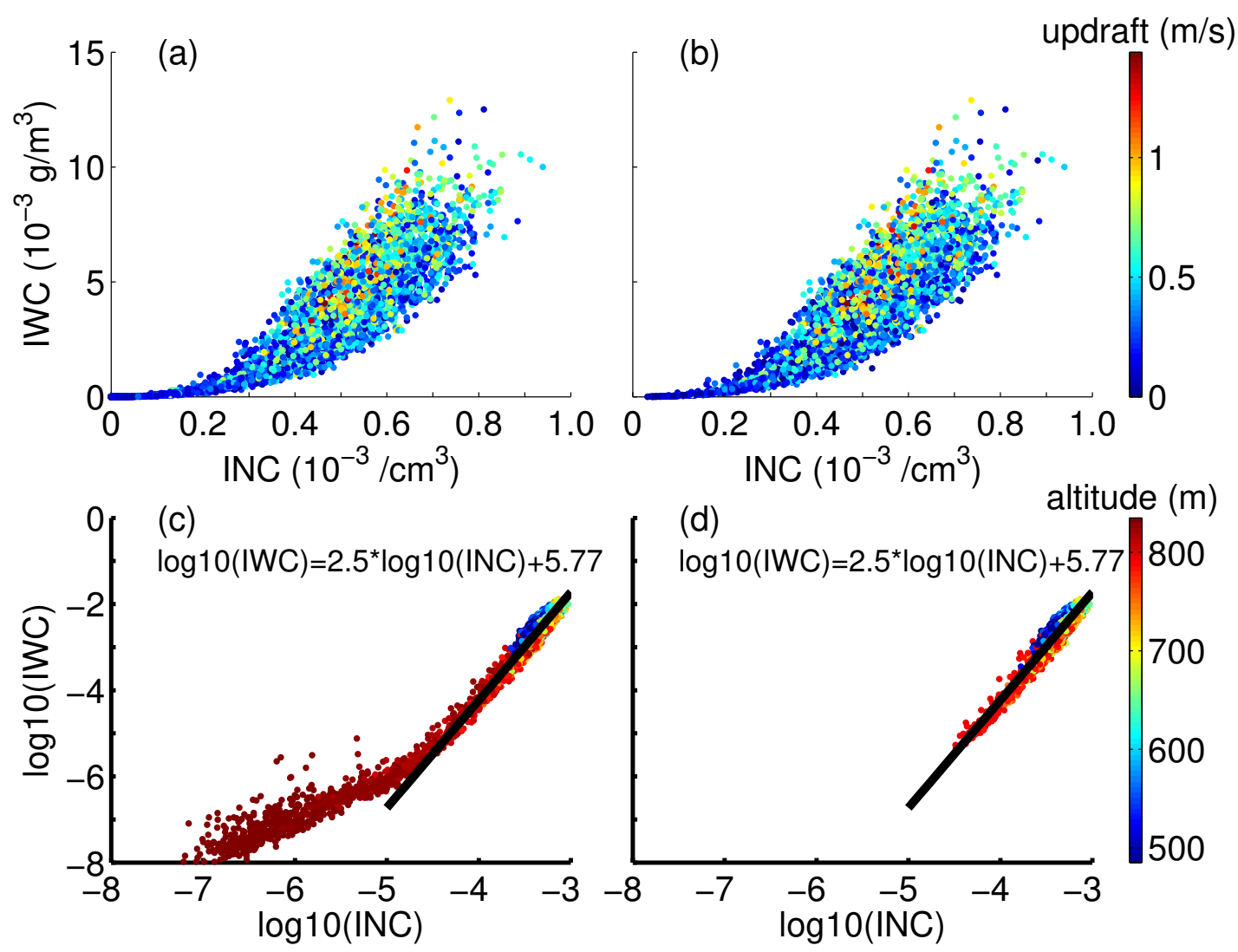

Figure 4.2: Ice water content and ice number concentration relationship from LES. (a) and (c) are accumulation zone region. (b) and (d) are selective accumulation zone region. Black lines in (c) and (d) are best fitted 2.5 slope lines. Colors in (a) and (b) represent updraft velocity, while colors in (c) and (d) mean altitude. The cloud base and top are at about $600 \mathrm{~m}$ and 800 $m$, respectively.

zone. Results are shown in Figure 4.2 (a) and (c). Each point represents the base of the accumulation zone for a single column in the LES. Colors in Figure 4.2 (a) represent the updraft velocity at that point, while colors in Figure 4.2 (c) represent the altitude of that point. Two power-law slopes clearly emerge in Figure 4.2 (c): One is the anticipated 2.5 slope and the other is the 1.0 slope expected for transport and dilution. In addition, we note that there is no preferred updraft velocity and altitude for the data on the line with 2.5 slope, whereas for data on the line with 1.0 
slope almost all points are at the top of the cloud. These columns actually contain only a small updraft at the cloud top, and the other part of the cloud is dominated by downdrafts. After removing these columns without a robust accumulation zone, we obtain a clear 2.5 power law between $w_{i}$ and $n_{i}$ (Figure 4.2 (b) and (c)). Further analysis of horizontal layers in the LES are shown in the supplementary material. Surprisingly, the 2.5 power law emerges not only in accumulation zones, but throughout the cloud, with the exception of just the cloud top region where entrainment and dilution is active and presumably the quasi-steady conditions are not reached.

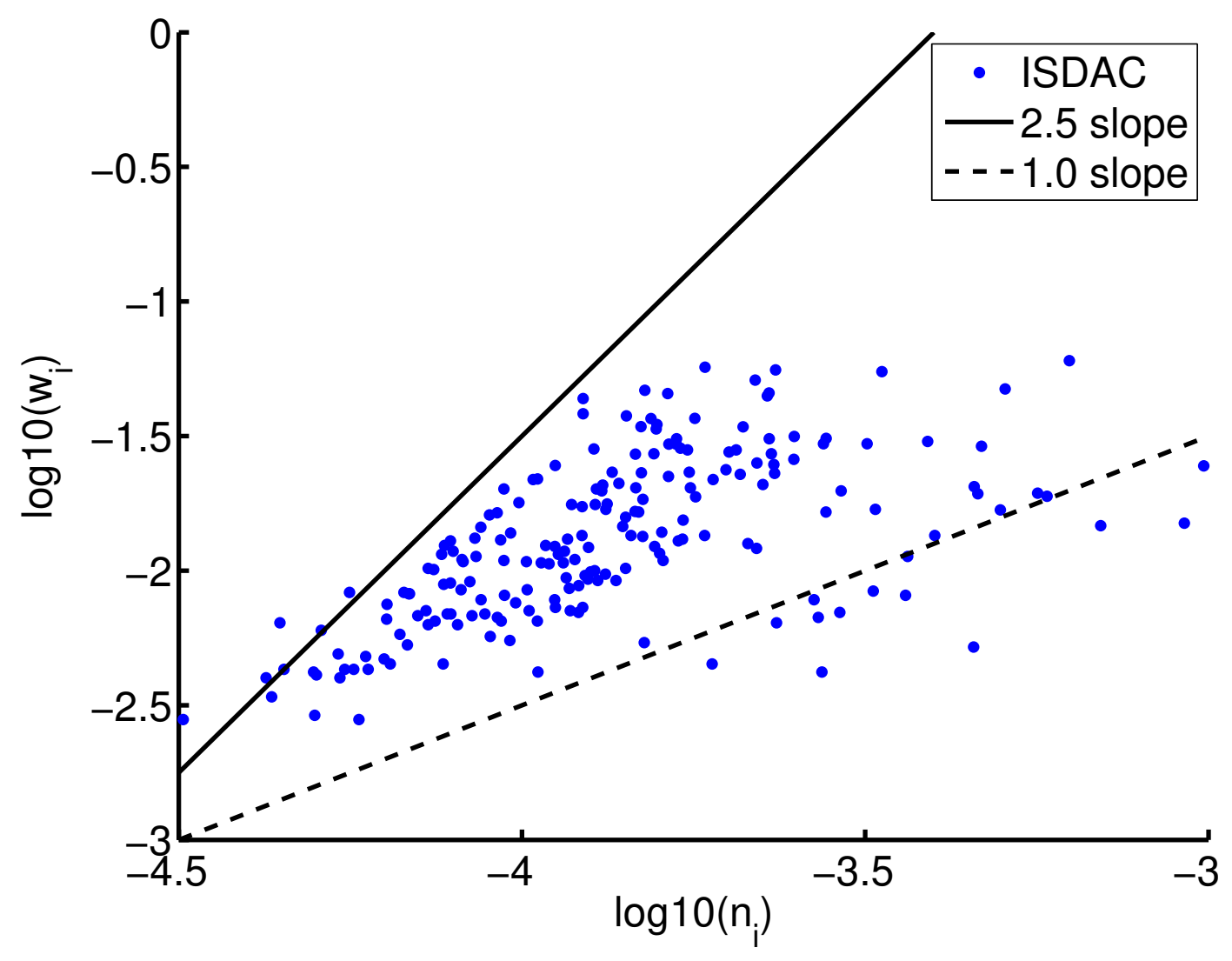

Figure 4.3: $w_{i}$ and $n_{i}$ relationship from Flight 31 ISDAC. Solid and dashed black lines represent 2.5 slope and 1.0 slope respectively. 
The LES observation of the 2.5 power law on horizontal layers allows us to consider whether there are similar indications in mixed phase clouds sampled during ISDAC. A full analysis is not possible in the space limitations of this letter, but here we use 1-s data from the 2DC and 2DP instruments taken during two horizontal in-cloud flight legs at around $700 \mathrm{~m}$ and $800 \mathrm{~m}$ on April 26 (the data set is discussed more fully by [29]). The data include ice particles larger than approximately $100 \mu \mathrm{m}$ in diameter and both $n_{i}$ and $w_{i}$ are derived from the size distributions. Obtaining reliable measures of $n_{i}$ are especially challenging due to ice crystal shattering, but the data have been post-processed to minimize such artifacts. Figure 4.3 shows the ISDAC data points plotted in log-log coordinates, and lines with slope 1.0 and 2.5 for comparison. Despite the measurement challenges, this first analysis suggests that the observed $\left(w_{i}, n_{i}\right)$ data lie within bounds set by the two power laws and therefore to the plausibility of the minimalist model assumptions.

Finally, we anticipate from Equation 4.5 that the intercept of the 2.5-slope line will be sensitive to the nucleation rate, being proportional to $\log \left(n_{i}^{\prime}\right)$. Figure 4.4 shows LES results for two ice nucleation rates: Blue points correspond to the LES with $\phi / \tau=2 * 10^{-9}$ and red points to $\phi / \tau=10^{-8}$. If we assume the correction parameter $\gamma$ doesn't change much between these simulations, the shift of the 2.5 slope line will be due only to a change in the ice nucleation rate. The intercept shift predicted by the minimalist model is $1.5 \log 10(5)=1.05$, which is very close to the best fitted line shift in Figure 4.4, $5.77-4.75=1.03$. Indeed, the two $\gamma$ values are 14.3 and 15.6 for 


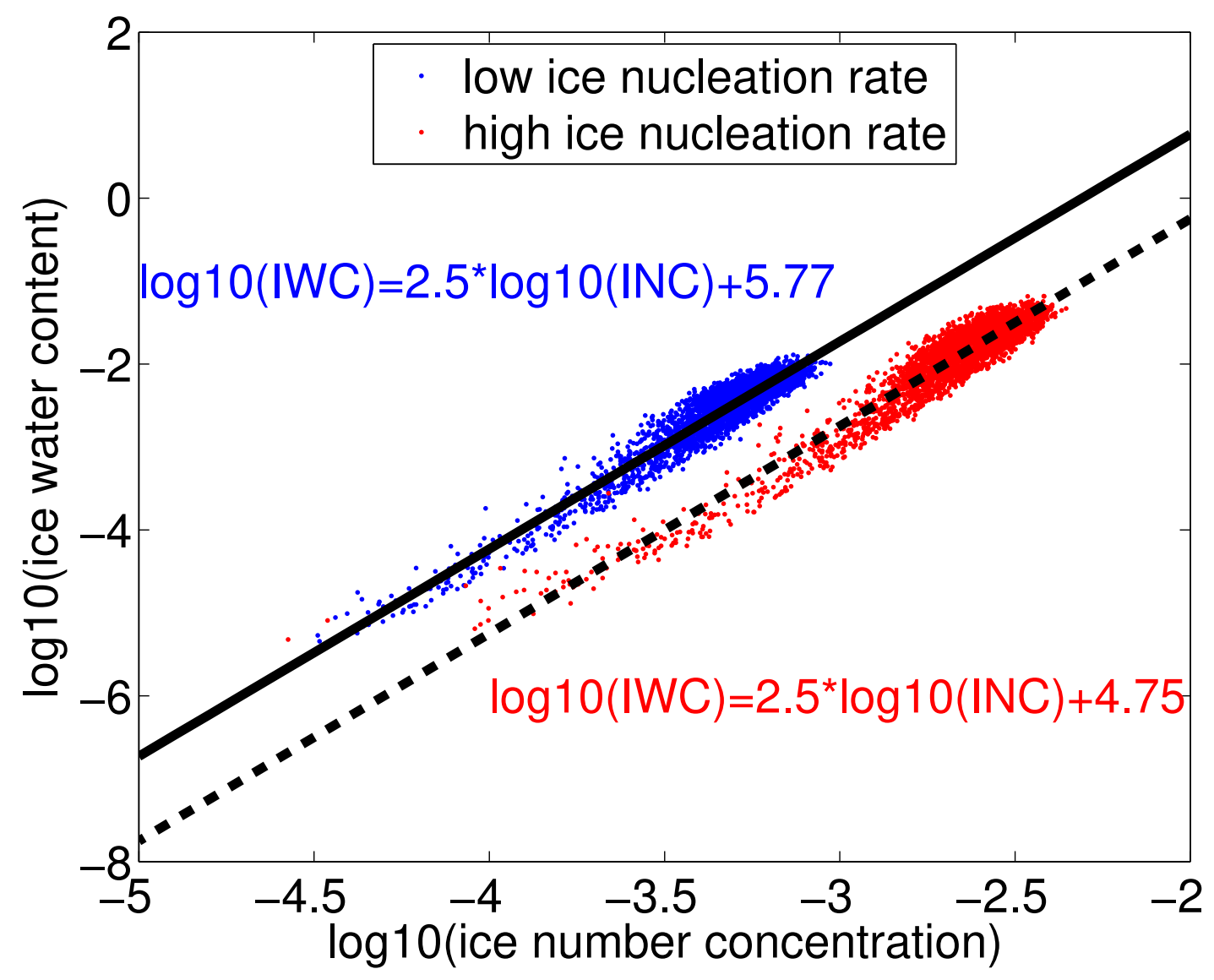

Figure 4.4: $w_{i}$ and $n_{i}$ relationship for two ice nucleation rates. Blue points are from LES with $\phi / \tau=2 * 10^{-9}$ and red points with $\phi / \tau=10^{-8}$. Solid and dashed lines are best fitted 2.5 slope lines.

low and high ice nucleation rates respectively(shape factor $C$ is set to be 1.0), so the assumption of constant $\gamma$ is reasonable. This provides a compelling link between ice microphysical properties and the ice nucleation rate within the cloud, which may be used in future analysis of cloud observations. 


\section{Conclusions}

We have approached the problem of steady ice precipitation from long-lived mixedphase clouds by assuming a steady state balance between new formation of ice particles due to a low stochastic ice nucleation rate throughout the cloud, and 'quasisteady' growth of ice particles by vapor deposition as they fall through updrafts and eventually out of the cloud base. A simple model based on this minimum number of assumptions is able to describe essential features of the ice microphysical properties of mixed-phase clouds. The model predicts a 2.5 power law between ice water content and ice number concentration in the cloud where updraft velocity decreases linearly with height. $w_{i}$ and $n_{i}$ from a LES cloud model with stochastic ice nucleation also follow the 2.5 power law, suggesting that the simple model can capture the properties of fully 3D mixed-phase cloud based on the assumption of plentiful, low efficiency ice nuclei and a stochastic ice nucleation process. Furthermore, a 2.5 power law relationship between $w_{i}$ and $n_{i}$ is observed as an upper bound in ISDAC measurements, suggesting the assumptions of the minimalist model are plausible and motivating additional analysis of ISDAC and other mixed-phase cloud data sets. These observations open up the intriguing possibility that ice microphysical properties within supercooled layer clouds can be used to investigate the nature of the ice nucleation process, and that similar models could serve as a bridge between complex field and cloud model observations and relatively idealized laboratory investigations of the time 
dependence of stochastic ice nucleation. The model also adds strength to the view that ice microphysics are tightly coupled with cloud dynamics and internal cloud variability, since the observed 2.5 power law is fundamentally tied to crystal growth in updrafts. Parameterizations based on these concepts are anticipated to be of value for larger-scale models in need of a physically based connection between cloud dynamics, ice nucleation, and the cloud microphysical properties that impact precipitation, cloud lifetime, and cloud optical properties.

\section{Acknowledgments}

This research was supported by the DOE Office of Science as part of the Atmospheric System Research program, including through grant DE-SC0006949, and used data from the Atmospheric Radiation Measurement Climate Research Facility. We thank A. Korolev for providing the processed ice data from ISDAC. Simulations were performed using PNNL Institutional Computing at Pacific Northwest National Laboratory. 


\section{Chapter 5}

\section{Microphysical Consequences Of}

\section{The Spatial Distribution Of Ice}

Nucleation In Mixed-Phase

\section{Stratiform Clouds}

This chapter details effect of spacial distribution of ice nucleation on the statistical properties of the mixed-phase stratiform cloud®1 This work was based on a research collaboration and is published in full form in the Geophysical Research Letters [149], and is reprinted with permission by the American Geophysical Union?2.

\footnotetext{
${ }^{1}$ This paper is co-authored by M. Ovchinnikov and R.A. Shaw

${ }^{2}$ Reprinted with permision from: F. Yang, M. Ovchinnikov and R.A. Shaw, Geophysical Research Letters, 41, 5280, 2014. Copyright 2014 by Geophysical Research Letters.
} 


\section{Abstract}

Mixed-phase stratiform clouds can persist even with steady ice precipitation fluxes, and the origin and microphysical properties of the ice crystals are of interest. Vapor deposition growth and sedimentation of ice particles along with a uniform volume source of ice nucleation, leads to a power law relation between ice water content $w_{i}$ and ice number concentration $n_{i}$ with exponent 2.5. The result is independent of assumptions about the vertical velocity structure of the cloud and is therefore more

general than the related expression of Yang et al. [2013] [148]. The sensitivity of the $w_{i}-n_{i}$ relationship to the spatial distribution of ice nucleation is confirmed by Lagrangian tracking and ice growth with cloud-volume, cloud-top, and cloud-base sources of ice particles through a time-dependent cloud field. Based on observed $w_{i}$ and $n_{i}$ from ISDAC, a lower bound of $0.006 \mathrm{~m}^{-3} \mathrm{~s}^{-1}$ is obtained for the ice crystal formation rate.

\section{Introduction}

Long-lifetime, mixed-phase stratiform clouds with continuous ice sedimentation are frequently observed in Arctic and mid-latitude regions [76, 132]. One question that remains unanswered is, what is the steady source of ice crystals? To compensate 
for the loss of ice nuclei due to ice particle sedimentation at cloud base, there must be a source of ice nuclei in the mixed phase cloud in order to maintain a steady state [137]. The source might be at the cloud top due to the evaporation nucleation or entrainment mechanism [13, 32], or in the whole cloud due to contact nucleation or time dependent stochastic ice nucleation [80, 137], or at the cloud base due to convection.

In a recent analytical model, [148] showed that a steady volume source for ice nucleation, as might be expected for stochastic ice nucleation, influences the microphysical structure of the cloud. Specifically, they argued that updrafts lead to 'accumulation zones' in which ice crystals can only be removed at the rate at which they grow by vapor deposition, with new ice crystals being constantly replenished through the steady ice nucleation rate. The resulting analytical model showed that ice water content $\left(w_{i}\right)$ and ice number concentration $\left(n_{i}\right)$ have a 2.5 power law scaling relationship,

$$
w_{i}=\frac{G n_{i}^{2.5}}{n_{i}^{\prime 1.5}},
$$

where $G=(4 / 3) \pi \rho_{i}\left(k C D s_{i}\right)^{1.5}$ with the units of $k g / s^{1.5} . n_{i}^{\prime}$ is the volume ice nucleation rate, $\rho_{i}$ is the density of ice, $C$ is the ice particle shape factor, $D$ is the modified diffusion coefficient, and $s_{i}$ is the water vapor supersaturation with respect to ice. The factor $k$ comes from the ice particle terminal velocity parametric equation [100], 


$$
v_{i}=b r_{i}^{k}
$$

where $r_{i}$ is the particle radius, and $b$ and $k$ were assumed to be constant parameters. A large eddy simulation (LES) cloud model with an idealized stochastic ice nucleation mechanism confirmed that $w_{i} \propto n_{i}^{2.5} / n_{i}^{\prime 1.5}$, but with a different prefactor. It was suggested that an empirical adjustment parameter to $G$ may be needed to compensate for the effect of three-dimensional (3D) dynamics in LES not captured by the idealized analytical one-dimensional (1D) model.

The work presented here is motivated by the following related questions. How sensitive are the ice microphysics in mixed-phase stratiform clouds to the spatial distribution of ice nucleation? For example, how general is the 2.5 power law between $w_{i}$ and $n_{i}$, and how will it differ for cloud top or cloud base ice nucleus seeding compared to volume based seeding? Finally, can $w_{i}-n_{i}$ observations allow for a quantitative determination of the ice nucleation rate $n_{i}^{\prime}$ in addition to indicating the spatial distribution of nucleation? The questions are addressed first by developing a new $w_{i}-n_{i}$ relationship for a steady, volume source of ice nucleation which is free of assumptions about the dynamic structure of the cloud. The conceptual and mathematical basis for the new approach are quite general, and therefore allow other ice nucleation sources, such as from cloud top, to be investigated. The analytical expressions are tested using the Lagrangian ice particle tracking method applied to the time dependent LES velocity 
field. Finally, the question of determination of $n_{i}^{\prime}$ is revisited, and a first estimate of the volume ice crystal formation rate is obtained for the Indirect and Semi-Direct Aerosol Campaign (ISDAC) Flight 31 case previously considered by [148].

\section{Analytical Model Of Ice Growth In A Mixed-Phase Stratiform Cloud}

The robust 2.5 power law observed between $w_{i}$ and $n_{i}$ in the LES cloud model results

of [148] came as somewhat of a surprise because the analytical result was obtained by assuming 'quasi-steady growth' in which ice particles are suspended in updrafts and only fall at the rate at which they grow by vapor deposition. Of course in a simulated 3D velocity field ice crystals can grow and fall out in downdraft regions, which will not satisfy the quasi-steady condition in the previous model. In this section we present an alternate model that does not rely on assumptions about the dynamical structure of the cloud. Essential assumptions are that the cloud has uniform temperature and possesses a large quantity of supercooled water so that the cloud humidity can be taken as saturated with respect to liquid water throughout. These assumptions are consistent with observations of long-lived, mixed-phase clouds that can be considered to be approximately in steady state, with uniformity of temperature and humidity valid for thin clouds. Ice crystals are allowed to nucleate at a temporally and spatially 
uniform rate within the cloud and to grow by vapor deposition as they settle through the cloud (aggregation is neglected).

We consider a mixed-phase cloud with a thickness of $h$ with steady volume ice nucleation rate $n_{i}^{\prime}$. Ice particle's terminal velocity is a function of radius $r_{i}$ described in Equation 5.2. In addition, the radius of an ice crystal $r_{i}$ will increase with time due to growth by vapor deposition as $d r_{i} / d t=C D s_{i} / r_{i}$, where $C$ is the shape factor, $D$ is a modified diffusion coefficient, and $s_{i}$ is the supersaturation with respect to ice. If $s_{i}$ doesn't change with time and we ignore the initial radius of the crystal,

$$
r_{i}^{2}=2 C D s_{i} t
$$

Thus the fall speed of ice crystal will increase with time,

$$
v_{i}=b r_{i}^{k}=b\left(2 C D s_{i} t\right)^{k / 2},
$$

and integrating over time results in an expression for the vertical distance traveled by the crystal,

$$
z_{i}=\frac{2}{k+2} b\left(2 C D s_{i}\right)^{k / 2} t^{(k+2) / 2} .
$$


It follows that the crystal falls a distance $z_{i}$ in time

$$
t=\left(\frac{k+2}{2 b\left(2 C D s_{i}\right)^{k / 2}} z_{i}\right)^{\frac{2}{k+2}} .
$$

We now consider growth of crystals falling from all levels within a mixed phase cloud with depth $h$ and vertical coordinate $z$ : at the cloud base $z=0$ and at the cloud top $z=h$. The number concentration at cloud base due to the crystals nucleated above within a narrow band of width $d z$ at level $z$ is $n_{i}^{\prime} d z / v_{i}(z)$, where $v_{i}(z)$ is the terminal velocity at cloud base of ice crystal generated within the band $d z$. So the ice number concentration $n_{i}$ at cloud base is,

$$
n_{i}=\int_{0}^{h} \frac{n_{i}^{\prime} d z}{v_{i}(z)} .
$$

The lower limit of integral 0 means particles are formed at cloud base and the upper limit of integral $h$ means seeding at the cloud top. Because $v_{i}$ is a function of $r_{i}$, while $r_{i}$ is related to $t$, and $t$ is a function of $z$, we can perform the integral analytically. The number concentration at cloud base resulting is

$$
n_{i}=\frac{k+2}{2} \frac{n_{i}^{\prime}}{\left((k+2) C D s_{i}\right)^{\frac{k}{k+2}}}\left(\frac{h}{b}\right)^{\frac{2}{k+2}} .
$$


Similar reasoning shows that the mass concentration at cloud base coming from a band of width $d z$ above cloud base is $m_{i}(z) n_{i}^{\prime} d z / v_{i}(z)$. Here, $m_{i}=\frac{4}{3} \pi \rho_{i} r_{i}^{3}$ is the single ice particle mass at cloud base generated in that band. As before, we can integrate over the depth of the cloud to obtain ice water content at cloud base

$$
w_{i}=\frac{k+2}{5} \frac{4}{3} \pi \rho_{i} n_{i}^{\prime}\left((k+2) C D s_{i}\right)^{\frac{3-k}{k+2}}\left(\frac{h}{b}\right)^{\frac{5}{k+2}}
$$

In order to get a height independent relationship between $w_{i}$ and $n_{i}$, we eliminate $h / b$ in Equations 5.8 and 5.9 to obtain,

$$
w_{i}=G^{\prime} \frac{n_{i}^{2.5}}{n_{i}^{\prime 1.5}}
$$

where $G^{\prime}=\frac{8}{15} \pi \rho_{i}\left(2 C D s_{i}\right)^{1.5}$. It should be noted that this relationship works not only at cloud base, but also at any level in the cloud (e.g., Equations 5.8 and 5.9 can be expressed for height $z$ by replacing $h$ with $h-z$, and then $(h-z) / b$ is eliminated to obtain Equation 5.10). Remarkably, the 2.5 power law emerges even without assumptions regarding the dynamical structure of the cloud that were made previously [148]. Furthermore, the result contains no dependence on parameters in the fall speed equation, $b$ and $k$. In both Equations 5.1 and 5.10 the relationship $w_{i} \propto n_{i}^{2.5} / n_{i}^{\prime 1.5}$ holds, with the prefactors related as $G^{\prime}=0.4(2 / k)^{1.5} G$. Finally, it is 
notable that in the current model ice particles at each level have a wide range of sizes, whereas they have the same size at cloud base in the idealized quasi-steady growth assumption in the previous model [148].

This result should be contrasted with the expectation for seeding from cloud top, under similar quiescent conditions. In that case we can write $n_{i}=n_{i, a}^{\prime} / v_{i}$ and $w_{i}=$ $(4 / 3) \pi \rho_{i} r_{i}^{3} n_{i, a}^{\prime} / v_{i}$, where $n_{i, a}^{\prime}$ is the ice nucleation rate per unit area through the cloud top. No integration is necessary in this case because the only source of ice crystals is at cloud top, and at least under the idealization of no turbulent mixing, all ice crystals therefore have the same history. Using Equations 5.3 and 5.4 we can directly obtain

$$
w_{i}=\frac{4}{3} \pi \rho_{i}\left(\frac{n_{i, a}^{\prime}}{b}\right)^{3 / k} n_{i}^{(k-3) / k} .
$$

It is immediately evident that, in contrast to Equation 5.10, this result contains no dependence on the vapor deposition rate, but instead depends directly on the parameters of the terminal fall speed relation (Equation 5.2). For large ice particles we can assume $k=1 / 2$, resulting in $w_{i} \propto n_{i, a}^{\prime 6} n_{i}^{-5}$. The -5 power law behavior is starkly different from the +2.5 power law behavior predicted by Equation 5.10 and by the approach of Yang et al. (2013) [148]. These results suggest that the vertical distribution of ice crystal formation in the cloud has major consequences for the ice 
microphysical properties within the cloud. In the following sections we explore these consequences.

\section{Lagrangian Ice Particle Tracking In LES Time De- pendent Field}

In order to investigate the expressions derived in previous section, namely to determine whether they capture essential behavior of ice microphysics in a dynamic, 3D velocity field, we use an large eddy simulation (LES). The velocity and thermodynamic fields and the liquid cloud microphysical properties are taken from the LES

cloud model [92], but ice crystal formation and growth is accomplished through a Lagrangian tracking method. The simulation is based on conditions measured during ISDAC Flight 31, as described by [91]; in that work it was shown that a supercooled liquid water cloud existed at water saturated conditions over many hours, with the relatively low ice concentration not significantly depleting that source. We perform the ice crystal tracking over the simulation time from 4 to 5.5 hours when the clouds are nearly in steady state conditions. Horizontally averaged profiles including temperature $(T)$, pressure $(p)$, liquid cloud fraction, relative humidity over liquid water $\left(R H_{w}\right)$ and ice $\left(R H_{i}\right)$ at the 4 hour simulation time, are shown in Figure 5.1, The liquid cloud fraction is near unity between $600 \mathrm{~m}$ and $800 \mathrm{~m}$, where $R H_{w}$ is about 
$100 \%$ and $R H_{i}$ is about $115 \%$.
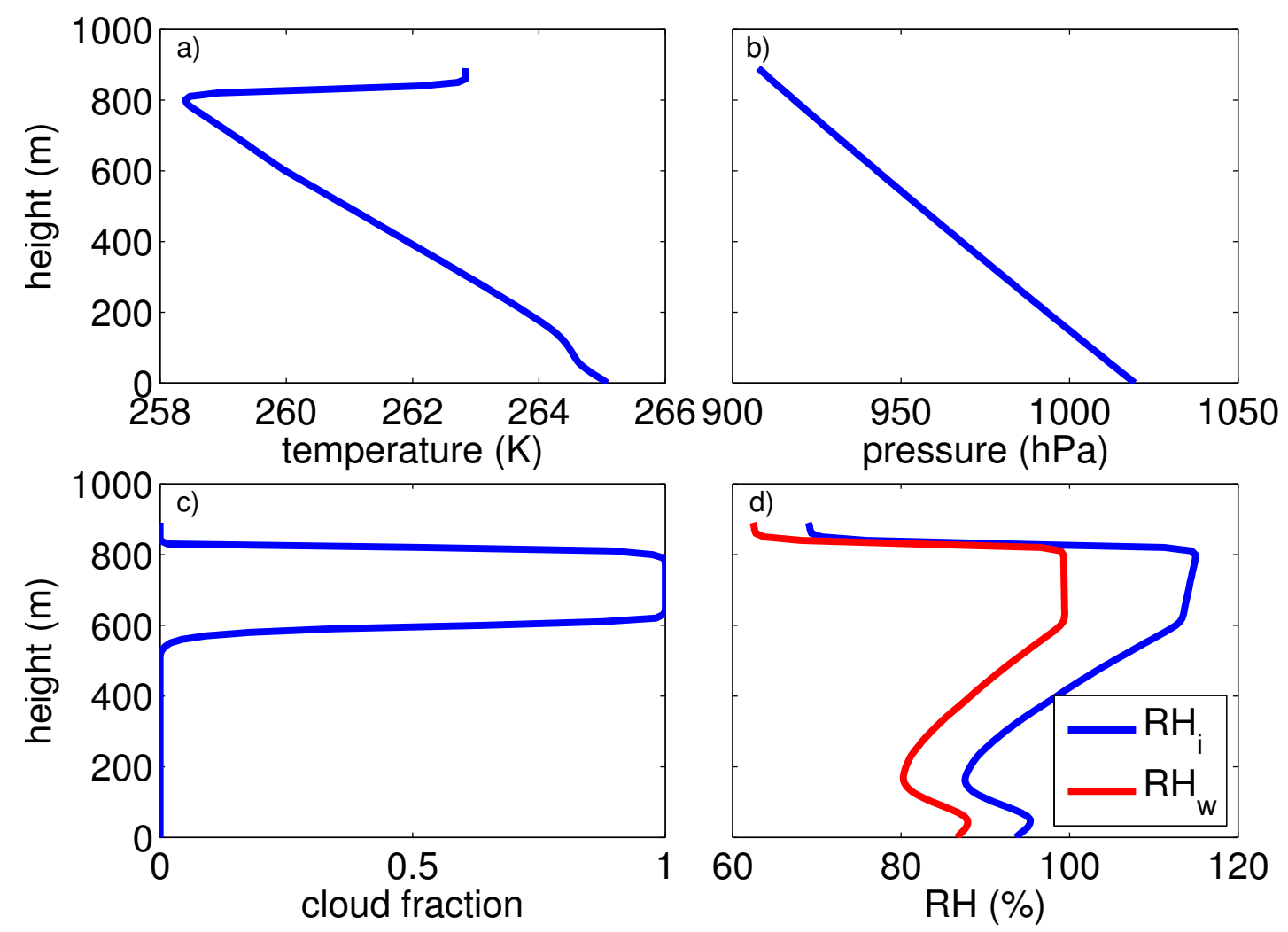

Figure 5.1: Mean vertical profiles of a) Temperature, b) Pressure, c) liquid cloud fraction and $\mathrm{d}$ ) relative humidity respective to liquid water and ice.

We seed ice particles in the liquid cloud layer, and consider the growth and sedimentation of ice particles in the time dependent LES field; velocities and thermodynamic properties are updated every 8 seconds. Ice particle trajectories are calculated using an Adams-Bashforth 2nd order method [42]. Ice particle terminal velocity is a function of its radius $v_{i}=17 r_{i}^{0.5}$, with $r$ in meters and $w_{t}$ in $m s^{-1}$ [91, 148]. The initial ice particle radius is set to be $10 \mu \mathrm{m}$. We assume ice particles are spherical and the growth of ice particles is only due to deposition of water vapor. The ice particle 
radius at time $t+d t$ is calculated via $r(t+d t)=\left(r(t)^{2}+2 C D s_{i} d t\right)^{0.5}$, where $d t$ is the modelling time step. For spherical ice particles, we set $C=1.0$ in this study. $s_{i}$ is calculated every 1 second as $s_{i}=\frac{e_{w}}{e_{i s}}$, where $e_{w}$ is the water vapor pressure and $e_{i s}$ is the saturated water vapor pressure over ice, which is a function of temperature.

We performed simulations with a volume distributed ice crystal source, and with area distributed sources (cloud top and cloud base, respectively), and in all cases the sources were assumed to be uniform and steady, based on the nearly-steady and stratiform conditions observed in the cloud. In each simulation we seed 81920 ice particles every 8 seconds throughout the cloud region. The three different locations are: (1) Uniform seeding in the liquid cloud layer (between $600 \mathrm{~m}$ and $800 \mathrm{~m}$ ) with $50 m$ horizontal separation and $10 m$ vertical separation. (2) Seeding at the cloud top region (between $798 \mathrm{~m}$ and $800 \mathrm{~m}$ ) with $50 \mathrm{~m}$ horizontal separation and 0.1 $m$ vertical separation (approximating an area source). (3) Seeding at the cloud base region (between $600 \mathrm{~m}$ and $602 \mathrm{~m}$ ) with $50 \mathrm{~m}$ horizontal separation and $0.1 \mathrm{~m}$ vertical separation (approximating an area source). The growth and sedimentation of ice particles are calculated along the trajectory of each ice particle in the time dependent field. If ice particles evaporate $\left(r_{i}<1 \mu m\right)$ or hit the ground, they are removed from the simulation and do not contribute to our final results. After 1.5 hours, the number of ice particles in one grid box represents $n_{i}$ in the units of \# per grid box, and the mass of ice particles in that grid box $\left(\sum \frac{4}{3} \pi \rho_{i} r_{i}^{3}\right)$ represents $w_{i}$, with units of $k g$ per grid box. Here we assume the density of ice particles is a constant $\rho_{i}=900 \mathrm{~kg} / \mathrm{m}^{3}$. 


\section{Influence Of The Spatial Distribution Of Ice Nucle- ation}

Using the ice crystal tracking within the simulated, time-dependent cloud, the dependence of ice microphysics on ice crystal seeding location can be investigated. For a uniform, steady-in-time source of ice crystals, consistent with the theory outlined in previous Section, the $w_{i}-n_{i}$ relationship at three different heights is shown in Figure 5.2. Each dot corresponds to $n_{i}$ and $w_{i}$ within a single grid box, and the cloud of dots in a given panel corresponds to all grid boxes at the specified height $z$. The lines correspond to Equation 5.1 (black line) and Equation 5.10 (red line). The green dot in each subplot corresponds to the horizontally averaged $\log \left(w_{i}\right)$ and $\log \left(n_{i}\right)$ for that layer. The previous model [148] captures the lower boundary of the points, while the new, generalized model captures the typical behavior and very closely coincides with the averaged properties. The spread of simulation points is generally captured by the predicted 2.5 slope, similar to the previously reported results from the LES with fully interactive dynamics and microphysics [148]. More careful statistical analysis suggests that the upper and lower boundaries of the data exhibit 1.0 and 2.5 slopes separately (see supplementary material). The upper boundary displaying a linear relationship between $n_{i}$ and $w_{i}$ is interpreted to result from turbulent mixing and dilution. [148] proposed that the 2.5 slope followed from the assumptions of "quasi-steady" vapor 
deposition growth as an ice crystal falls slowly through an updraft and a steady, volume source of ice crystal nucleation. We see now that, although the updraft may be necessary to predict maximum ice crystal sizes (as was the original motivation in the previous work), the majority of the cloud has ice microphysical properties that depend simply on the assumptions of vapor deposition growth as ice crystals fall at terminal speed, and a steady, volume source of ice nucleation.
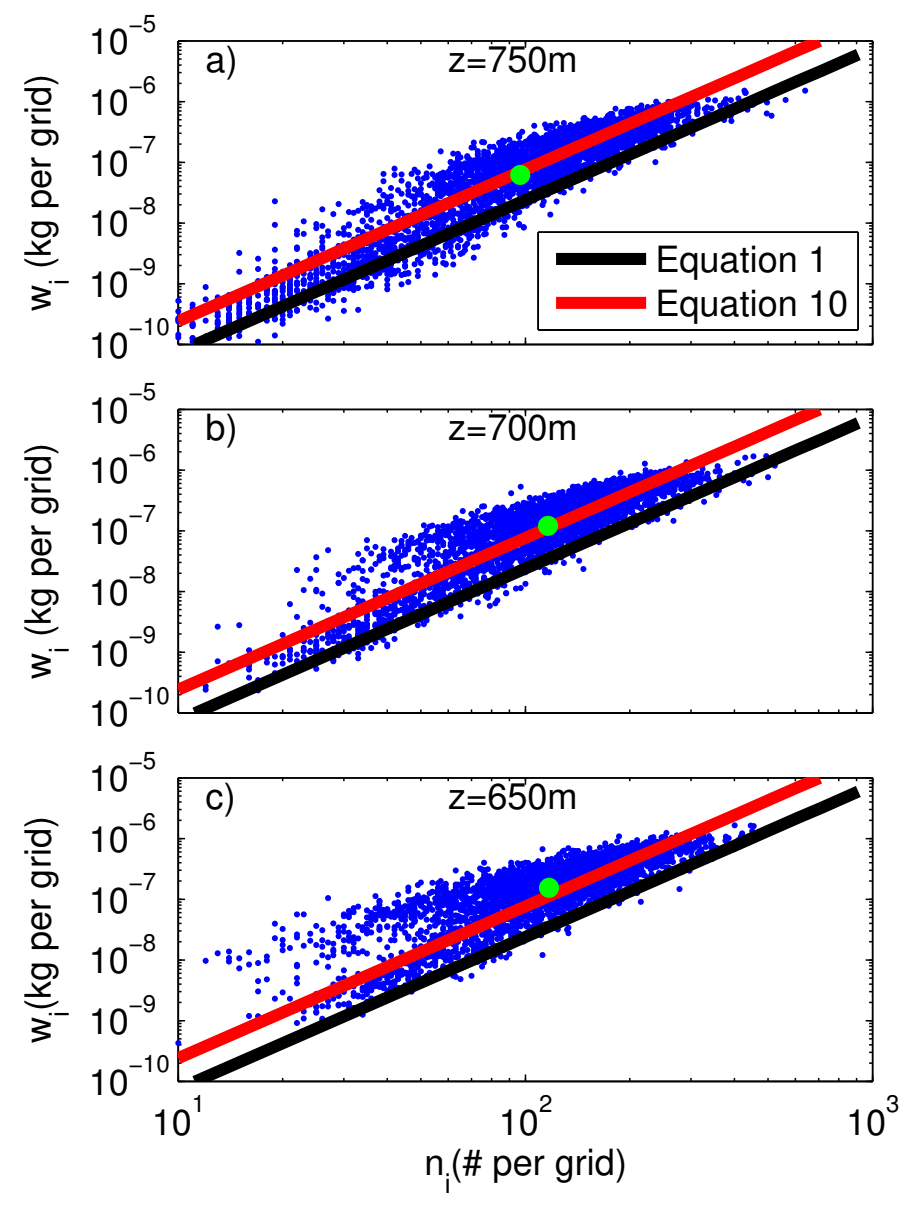

Figure 5.2: $w_{i}$ and $n_{i}$ relationship at three different layers from the Lagrangian particle tracking method in LES time dependent field. Black line is based on Equation 5.1 and red line is from Equation 5.10. Green dot corresponds to the average $\log \left(n_{i}\right)$ and $\log \left(w_{i}\right)$ at each level. 
To test whether the ice crystal seeding distribution affects the microphysical properties and the $w_{i}-n_{i}$ power law as implied by Equation [5.11, we carried out a simulation with ice crystals formed at cloud top. Finally, for sake of completeness we carried out a simulation with ice crystals formed at cloud base. No theory is provided for this latter scenario because it would necessarily rely on dynamical assumptions (i.e., updraft velocity is necessary to carry ice into the supercooled liquid cloud). Figure 5.3 shows the $w_{i}-n_{i}$ relationship for the three seeding locations (the volume-source results are repeated for direct comparison) at three different levels $(650 \mathrm{~m}, 700 \mathrm{~m}$ and $750 \mathrm{~m}$ ). The red line in each subplot is calculated from Equation 5.10. For comparison the brown dash lines have slope 1.0 as would be expected for simple dilution and turbulent mixing, i.e., $n_{i}$ and $w_{i}$ increasing or decreasing in direct proportion. In the middle-column panels, corresponding to ice crystal seeding at cloud top, the grey line displays a slope of -5 as expected from Equation 5.11 .

The simulation results validate the theoretical expectation that the 2.5 power law appears in the uniform volume-seeding case (Figure $5.3 \mathrm{a}, \mathrm{d}, \mathrm{g}$ ), but disappears for both cloud-top seeding (Figure $5.3 \mathrm{~b}, \mathrm{e}, \mathrm{h}$ ) and cloud-base seeding (Figure $5.3 \mathrm{c}, \mathrm{f}, \mathrm{i}$ ). The cloud-top seeding results indeed suggest a spread of points along the -5 slope line, but it should be pointed out that the prefactor given in Equation 5.11 does not predict the proper location of the line, presumably due to the strong 3D effects of turbulence. Turbulent mixing and dilution play a strong role in determining the ice microphysical properties in the cloud-top and cloud-base simulations as evidence by 


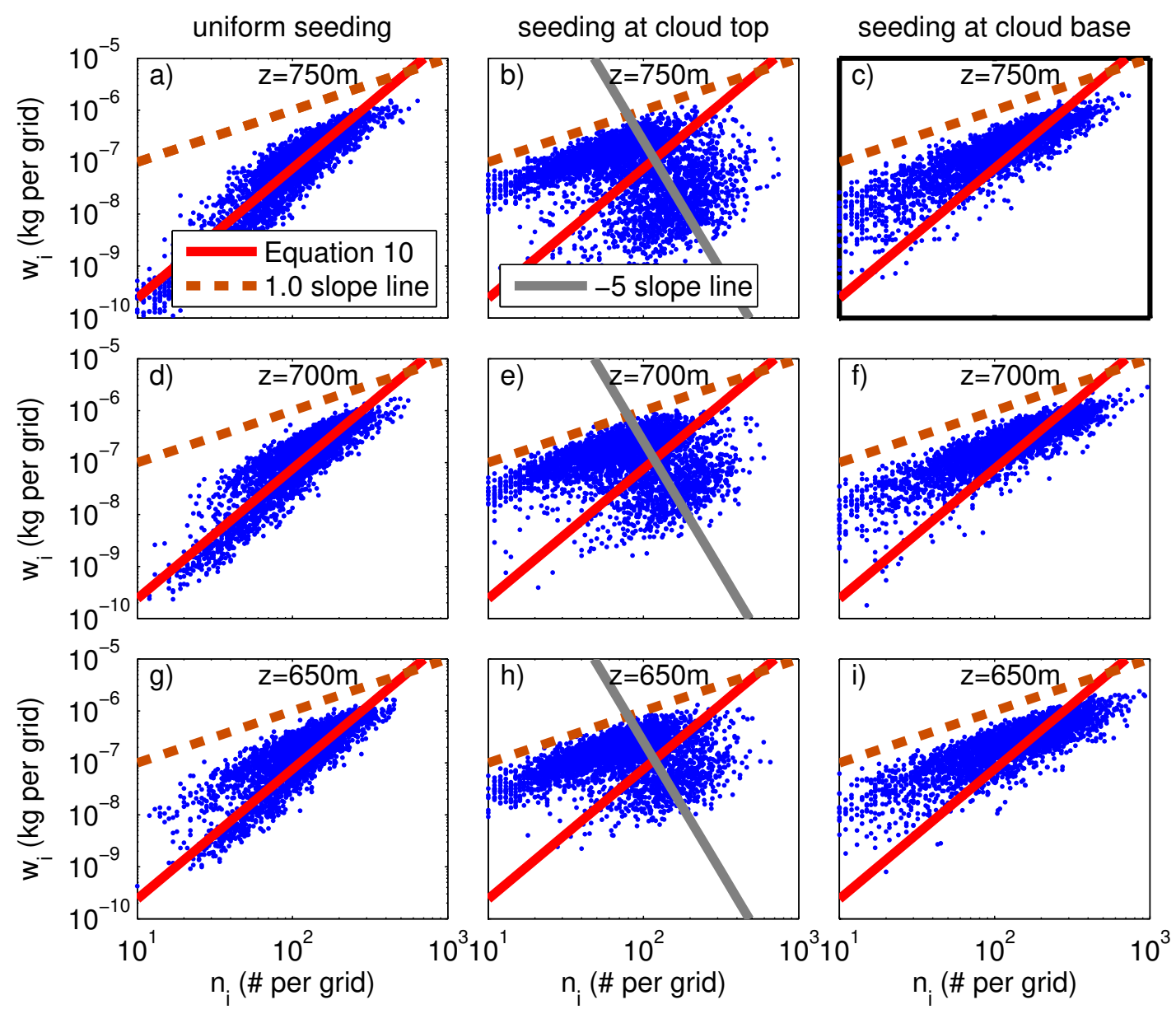

Figure 5.3: Relationship between $w_{i}\left(\mathrm{~kg}\right.$ per grid) and $n_{i}$ (\# per grid) at $650 m$ (g,h,i), $700 m$ (d,e,f) and $750 m$ (a,b,c) for three different seeding places.a), d) and g) are uniform seeding in the cloud layer; b), e) and h) are seeding at cloud top; c), f) and i) are seeding at cloud base. Red line is calculated from Equation 5.10, and brown dash line is 1.0 slope line, while grey line is -5 slope for comparison.

the clear distribution of points along 1.0-slope lines. There is also a formation of a 1.0-slope branch of points at low levels in the uniform volume-seeding case (panel g).

In the previous work ISDAC data was shown that provided some support for the appearance of a 2.5 slope in naturally occurring clouds [148], but no quantitative 


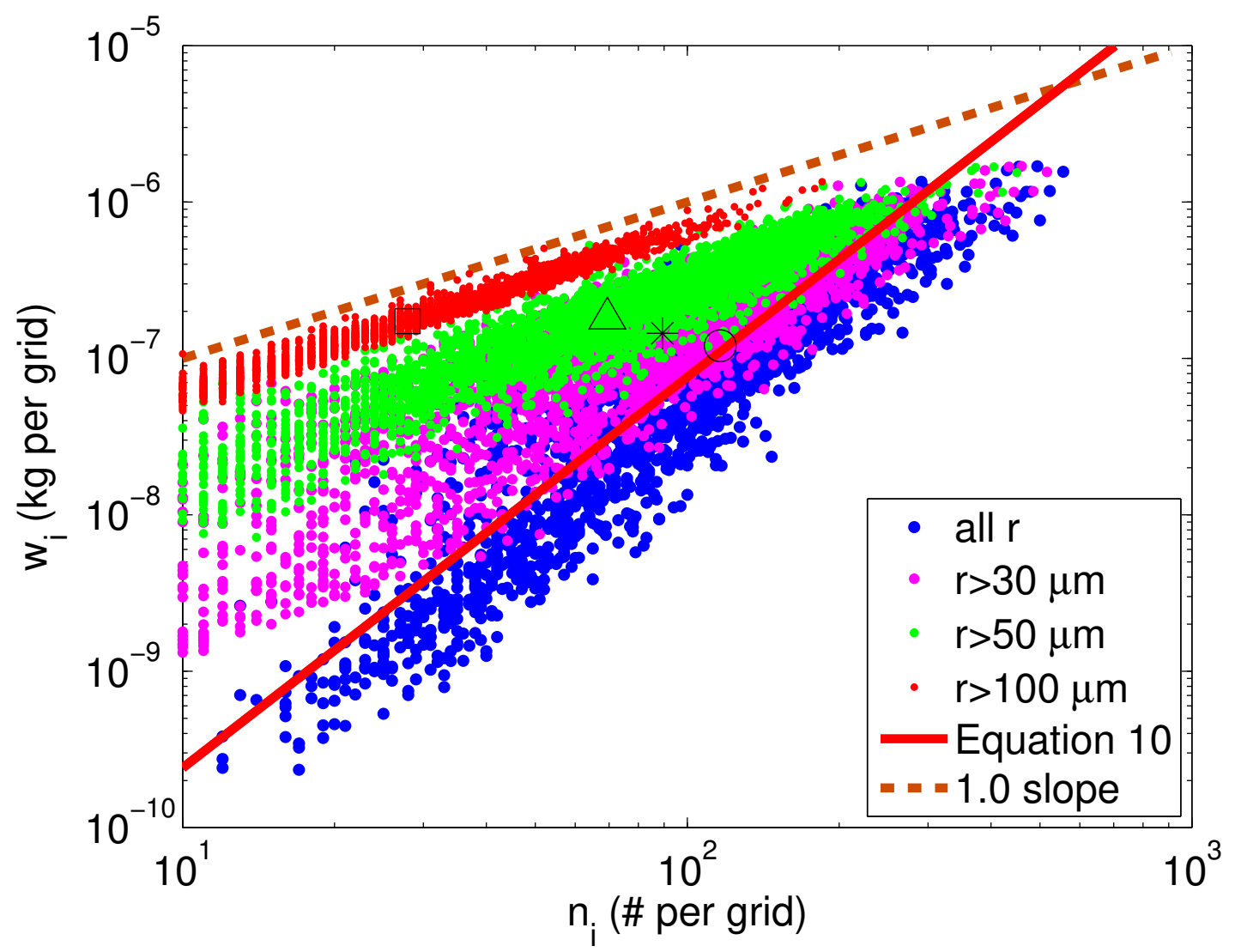

Figure 5.4: Relationship between $w_{i}\left(k g\right.$ per grid) and $n_{i}$ (\# per grid) at $700 \mathrm{~m}$ for different threshold radii from the Lagrangian particle tracking model. Red line is calculated from Equation 5.10 and the brown dash line is 1.0 slope for comparison. Circle, star, triangle and square represent the average of $\log \left(w_{i}\right)$ and $\log \left(n_{i}\right)$ for blue, pink, green and red dots separately.

conclusions were drawn at that stage. For a simple closure experiment, we estimate $n_{i}^{\prime}$ from the average $w_{i}$ and $n_{i}$ data in Figure 5.2, Results show that $n_{i}^{\prime}$ calculated from Equation 5.10 are 0.10, 0.12 and 0.14 \# per grid per second at 650, 700 and 750 $m$ separately, which are close to the exact $n_{i}^{\prime}=0.125 \mathrm{~s}^{-1}$ (The calculation is made in log-log coordinates; the value of 0.125 arises from the imposed nucleation rate of one ice crystal per grid per LES time step). Given the success of Equation 5.10 in describing the LES results in previous Section we can ask whether it is possible to 
obtain an estimate of the volume ice nucleation rate $n_{i}^{\prime}$ in a mixed phase cloud. The estimate of $n_{i}^{\prime}$ would be made by calculating the mean $\overline{w_{i, o b s}}$ and $\overline{w_{i, o b s}}$ in the $\log -\log$ coordinates, then $n_{i}^{\prime}=\left(G^{\prime}{\overline{n_{i, o b s}}}^{2.5} / \overline{w_{i, o b s}}\right)^{1 / 1.5}$ based on Equation 5.10. We emphasize that the estimation of $n_{i}^{\prime}$ only implies a steady volume ice crystal formation rate, and does not necessarily confirm details about whether that is due to immersion nucleation based on a stochastic ice nucleation mechanism, contact nucleation based on a stochastic collection process, or some other mechanisms. The previous study showed that the observed $w_{i}$ and $n_{i}$ from ISDAC Flight 31 are bounded by 2.5 and 1.0 slope lines (see Figure 4.3). For the first approximation, the average $\log \left(w_{i}\right)$ and $\log \left(n_{i}\right)$ are calculated as $-1.9\left(w_{i}=0.0141 \mathrm{gm}^{-3}\right)$ and $-3.9\left(n_{i}=0.126 \mathrm{~L}^{-1}\right)$. Based on Equation 5.10, we can estimate the volume ice nucleation rate to be $n_{i}^{\prime} \approx 0.006$ $m^{-3} s^{-1}$. It should be noticed that Equation 5.10 assumes that ice particles of all possible radii are present, while data from observation have a threshold value due to the instrument resolution limitations. The influence of a threshold radius on the $w_{i}-n_{i}$ relationship from the Lagrangian particle tracking model is investigated in Figure 5.4. It can be seen that the 2.5 power law slope is gradually masked as the threshold radius is increased, with the slope eventually making a transition to close to 1.0 at larger threshold radius. In addition, a larger threshold radius will bias the measurements of $w_{i}$ and $n_{i}$ and therefore lead to lower estimates of $n_{i}^{\prime}$. From the LES example in Figure 5.4 the estimated $n_{i}^{\prime}$ based on the average of $\log \left(w_{i}\right)$ and $\log \left(n_{i}\right)$ is 0.12 for all data, but is only $0.067,0.038$ and 0.0086 for threshold radii of $30 \mu \mathrm{m}, 50 \mu \mathrm{m}$ 
and $100 \mu m$, respectively. This suggests that values estimated from measurements, such as the $0.006 \mathrm{~m}^{-3} \mathrm{~s}^{-1}$ obtained from ISDAC data, could be one or even up to two orders of magnitude smaller than the true value. Detailed comparison with data will require additional effort, for example because the relationship between equivalentsphere radius and ice crystal maximum dimension is shape dependent, and therefore the instrument resolution effect will be more complex. In addition, uncertainty in the ice crystal density and shape factor, as well as in the modified water vapor diffusion coefficient (which includes effects of ice accommodation coefficients) will also affect the estimation of $n_{i}^{\prime}$.

\section{Conclusions}

Under steady state conditions, a mixed phase, stratiform cloud will exhibit microphysical properties that are surprisingly sensitive to the spatial distribution of ice crystal formation. We have shown that a volume-distributed source of ice nucleation quite generally exhibits a 2.5 power law slope between $w_{i}$ and $n_{i}$. The 2.5 slope arises directly from expressions for vapor-deposition growth and sedimentation of ice particles along with a steady volume source of ice crystals. Physically, once ice particles are formed, they will grow and fall at the same time. As they fall to lower levels, new ice particles are continuously formed form there. In other words, at a specific 
height, different ice particles experience a variety of histories. This integral distribution leads to the 2.5 power law. Both theory and simulation show that the 2.5 power law breaks down for other spatial distributions of ice nucleation; for example, the same theoretical approach gives a power law slope of -5 for a cloud-top source of ice nucleation. In simulations with both cloud-top and cloud-base ice nucleation a slope of 1.0 is strongly evident, and it is even apparent in the spread of points for the volume source of ice nucleation. This linear relationship between $w_{i}$ and $n_{i}$ can be interpreted as resulting from simple dilution or turbulent mixing between cloud regions with different histories. We summarize the main findings of this work as follows:

$\dagger$ The 2.5 power law relationship between $w_{i}$ and $n_{i}$ predicted by the analytical model presented here can be considered more general than the result obtained previously [148]. Specifically, the approaches have the following differences: (1) The old model rested on the assumption of a linearly-decreasing updraft velocity profile, while the new model does not include explicit assumptions about the dynamical structure of the cloud. (2) Ice particles at one single layer based on the old model have only one size, while in the model presented here there is a broad size distribution for ice particles. (3) The new approach quantitatively predicts the average of $w_{i}$ and $n_{i}$ from the LES Lagrangian tracking model, while the old model was only able to describe a boundary for the simulation data. 
$\dagger$ This 2.5 power law relationship arises directly from vapor-deposition growth and sedimentation of ice particles along with a steady volume source of ice crystals. Without the growth of ice particles, $w_{i}$ and $n_{i}$ have a linear relationship; furthermore, turbulent mixing and dilution tend to favor such a linear relationship. Simulations confirm that without continuous and uniform seeding throughout the whole cloud volume, e.g. seeding of ice crystals from cloud top or cloud base, the 2.5 power law relationship disappears. A steady, uniform source of ice crystals from cloud top leads, for an idealized non-turbulent cloud, to a prediction of a -5 power law between $w_{i}$ and $n_{i}$. Simulations with cloud top seeding exhibit a spread of points that appears consistent with this scaling, along with spread along +1.0 lines. The power law approach is very general and avoids difficulties in matching cloud height, for example; future work will address topics such as the vertical profiles predicted by Equations 5.8 and 5.9.

† Observational data have shown some evidence for the 2.5 power law relationship, which means that the steady volume ice nucleation rate might exist. As a first approximation, we estimate $n_{i}^{\prime}$ in the mixed phase cloud to be approximately $0.006 \mathrm{~m}^{-3} \mathrm{~s}^{-1}$ based on the observed $w_{i}$ and $n_{i}$ from ISDAC Flight 31 . Increasing the threshold radius due to the observation equipment could decrease both the power law slope from 2.5 to 1.0 and the estimated $n_{i}^{\prime}$ by one to two orders of magnitude. To make a better estimation of $n_{i}^{\prime}$, density, shape factor, and accommodation coefficients of ice particles in the mixed phase cloud should be 
known more accurately, and a detailed analysis of the instrument size threshold for measurement of $w_{i}$ and $n_{i}$ should be performed.

In the larger context, these results have several possible implications and applications. First, this generalized model for $w_{i}-n_{i}$ statistics provides an alternate method for investigating the ice nucleation process. For example, if $w_{i}$ and $n_{i}$ measurements are observed to robustly exhibit the 2.5 power law relationship, it is a strong indication for a uniform volume source of ice nucleation throughout the whole cloud. This in turn provides hints for the ice nucleation mechanism, such as immersion nucleation with a stochastic ice nucleation rate or perhaps contact nucleation based on a stochastic collection process. Second, even without details of the mechanism, this model provides a basis for indirectly determining the ice crystal formation rate $n_{i}^{\prime}$ quantitatively. This quantity is important for determining the stability and properties of mixed-phase clouds, and in principle could be investigated over a variety of thermodynamic and environmental conditions using direct or remote measurements of ice microphysical properties. Third, because this model is based on simple assumptions (e.g., prescribed spatial distribution for ice formation) and established theoretical expressions (e.g., terminal fall speed, vapor deposition growth rate), it may provide a basis for developing mixed phase cloud parameterizations useful in coarse-grained computational models. 


\section{Acknowledgements}

This research was supported by the DOE Office of Science as part of the Atmospheric

System Research program, including through grant DE-SC0006949, and used data

from the Atmospheric Radiation Measurement Climate Research Facility. Simulations were performed using PNNL Institutional Computing at Pacific Northwest National Laboratory. 



\section{Chapter 6}

\section{Long-Lifetime Ice Particles In}

\section{Mixed-Phase Stratiform Clouds:}

\section{Quasi-Steady And Recycled}

\section{Growth}

This chapter details the existence of long-lifetime ice particles in the mixed-phase

stratiform cloud\$1 This work was based on a research collaboration and is published in full form in the Geophysical Research Letters [150], and is reprinted with permission by the American Geophysical Union2 2 .

${ }^{1}$ This paper is co-authored by M. Ovchinnikov and R.A. Shaw

${ }^{2}$ Reprinted with permision from: F. Yang, M. Ovchinnikov and R.A. Shaw, Journal of Geophysical Research: Atmospheres, 120, 2015. Copyright 2015 by Geophysical Research Letters. 


\section{Abstract}

Ice particles play an important role in precipitation and radiation transfer in stratiform mixed-phase clouds. Lagrangian ice particle tracking in mixed-phase clouds is applied in both a 3-D time dependent velocity field produced by a Large Eddy Simulation cloud model and in a 2-D idealized field. It is found that more than $10 \%$ of ice particles have lifetimes longer than 1.5 hours, much longer than the large eddy turnover time or the time for a crystal to fall through the depth of a non-turbulent cloud. An analysis of trajectories in a 2-D idealized field shows that there are two types of long lifetime ice particles: quasi-steady and recycled growth. For quasisteady growth, ice particles are suspended in the updraft velocity region for a long time. For recycled growth, ice particles are trapped in the large-eddy structures, and whether ice particles grow or evaporate depends on the ice relative humidity profile within the boundary layer. Some ice particles can grow after each cycle in the trapping region, until they are too large to be trapped, and thus have long lifetimes. The relative contribution of the recycled ice particles to the cloud mean ice water content depends on both the dynamic and thermodynamic properties of the mixing layer. In particular, the total ice water content of a mixed phase cloud in a decoupled boundary layer can be much larger than that in a fully coupled boundary layer. 


\section{Introduction}

Thin, stratiform, mixed-phase clouds cover large portions of the midlatitudes and polar regions. They are radiatively important and can play an integral part in the evolution of boundary layer structure and surface fluxes in sensitive areas like ice covered oceans [20, 78, 132]. The observation that ice is often generated in these clouds continuously over long time is perhaps surprising, and efforts have been made to understand origins of the ice [33, 80, 137, 148].

Microphysical properties of mixed phase clouds, such as ice number concentration, ice water content, liquid-ice water partition, play an important role in their radiative properties, precipitation efficiency and cloud lifetime [52, 89]. Recently, a minimalist model predicted that the ice number concentration and ice water content has a general 2.5 power law relationship, if the new ice particle nucleation rate is a constant [149]. Data from a large eddy simulation (LES) cloud model confirmed the 2.5 power law relationship, and observation data also shows a similar non-linear trend. How-

ever, this simple model cannot explain the existence of large ice crystals that have been found in both observational and modelling studies. For example, observational data from Flight 31 of Indirect and Semi-Direct Aerosol Campaign (ISDAC) show that the mean maximum ice particle dimension at mixed phase cloud base can be 
larger than $1 \mathrm{~mm}$, while its mass-equivalent spherical ice particle diameter is around $600 \mu \mathrm{m}$ [76]. Data from the LES cloud model with bin microphysics scheme also captures those large ice particles [91], but without Lagrangian information it is difficult to identify the mechanisms for their formation. Using the analytical model, the maximum radius for an ice crystal to fall out of the mixed phase cloud in a still environment (no updraft or downdraft) can be calculated, assuming the ice crystal is initially located at cloud top: we assume the initial ice particle size is $10 \mu m$ radius, cloud top is at $800 \mathrm{~m}$, liquid cloud base is at $600 \mathrm{~m}$, and the supersaturation with respect to ice within the mixed phase cloud region is $10 \%$. The growth of the ice particle's radius follows $r=\left(r_{0}^{2}+2 C D s_{i} t\right)^{0.5}$, where $r_{0}$ is the initial ice particle radius, $C$ is the shape factor, $D$ is the modified diffusion coefficient, $s_{i}$ is the supersaturation over ice and $t$ is time. The ice particle is assumed to fall at terminal velocity, described by the equation $v=17 r^{0.5}[149]$. Combining the above two equations, we can numerically or analytically calculate the maximum ice particle diameter at mixed phase cloud base, obtaining approximately $200 \mu \mathrm{m}$. This size is much smaller than that from the ISDAC observational data as we discussed before. Our size estimation depends on a number of simplifications in formulations of diffusional growth and fall velocity, and neglecting collisions among ice particles. However, the same approximations of ice processes were used in an LES cloud model, which predicted ice crystal sizes comparable to ISDAC observations. This indicates that there might be some some dynamical factors that allow ice particles to persist for longer times 
and grow larger in a realistic mixed phase cloud than in an idealized still environment.

Previous research has shown that the production of large drops is related to the dynamic motions in the boundary layer [31]. It is also believed that the recycling of ice particles in thunderstorms is the key mechanism for hail formation [10]. In addition, aerosols such as cloud condensation nuclei (CCN) and ice nuclei (IN) can be transported through cloud. Even when cloud droplets totally evaporate, CCN/IN can be activated again due to recycling [30, 118]. Aerosol chemical and physical properties can change during the recycling processes, which might have an effect on cloud microphysics and climate [1, 130]. There is awareness that eddies can recycle falling particles, and therefore might affect their lifetimes, but, to our knowledge, implications of this process for ice particles in stratiform mixed phase clouds have not been qualitatively studied.

This chapter builds on the effort of [149] by considering the mechanisms for producing large ice crystals in shallow, mixed-phase stratiform clouds, drawing attention to the role of the boundary layer dynamical structure. We adopt a Lagrangian approach to investigate the lifetime and size evolution of ice crystals in a turbulent boundary layer. First, we analyze the statistics of ice crystal lifetimes in a three-dimensional, dynamic field from a detailed large-eddy simulation model. In order to obtain 
a physical understanding of the processes involved, we then analyze ice crystal trajectories in an idealized two-dimensional kinematic velocity field and develop analytical solutions for ice crystal orbits. In addition, we consider how the turbulent and thermodynamic structure of the boundary layer influence the generation of large ice crystals and determine the mixed-phase cloud ice water content. Finally, we discuss implications and summarize the results.

\section{Model Setup For Langrangian Ice Particle Tracking In 3D Time Dependent Field}

In order to investigate the production of a small number of large ice crystals, we analyze the growth of ice crystals within a simulated mixed-phase stratiform cloud. Growth is calculated along Lagrangian tracks of crystals within the dynamic, three-dimensional velocity and thermodynamic fields generated by a LES of an Arctic springtime cloud-topped boundary layer. The LES model and simulation setup based on observations from ISDAC Flight 31 [76] are described in detail by

[91, 92], and only their main features are briefly summarized below. The LES model is based on the System for Atmospheric Modeling (SAM, version 6.10.3), which is built on an analestic dynamical framework and uses liquid-ice moist static energy 
and total water specific humidity as prognostic thermodynamical variables [55]. Mass and number mixing ratios for cloud droplets and ice particles are predicted by the microphysics scheme [77] with the following important modifications. Only one category of ice particles is considered, and mass-size and mass-fall speed relationships are chosen to be in the form of power laws representative of dendrite crystals, - the dominant crystal type observed during the studied case. The size distribution of ice crystals is treated as a Gamma distribution with the shape parameter 3, which has been shown to more closely match the cloud properties computed with more sophisticated size-resolved microphysics treatments than using the default shape parameter value of 0 for an exponential size distribution [91]. Collision-coalescence process is neglected and the ice nucleation parameterization is replaced by a simple relaxation scheme that keeps the ice number mixing ratio tied to the prescribed value $\left(1 g^{-1}\right)$. The longwave radiative cooling is parameterized as a function of the liquid water content profile and shortwave radiation is neglected.

The three-dimensional model domain covers $6.4 \mathrm{~km}$ in both horizontal directions and $1.6 \mathrm{~km}$ in the vertical, with grid spacings of $50 \mathrm{~m}$ and $10 \mathrm{~m}$, respectively. Lateral boundary conditions are cyclic and surface latent and sensible heat fluxes are set to zero. Large scale subsidence is prescribed at $0.4 \mathrm{~cm} \mathrm{~s}^{-1}$ above and at the capping inversion level $(z=825 \mathrm{~m})$ and is decreasing linearly toward the surface. The initial sounding contains a well-mixed layer extending from altitude 
of $400 \mathrm{~m}$ to the inversion and a slightly moister and more stable layer below 400 $m$. Additional details of and justification for the presented setup are provided by [91].

The simulation is run for eight hours with the second half of the run being approximately in steady state. LES velocity and thermodynamics fields archived over hours 4 and 5 are used in the Lagrangian particle-tracking model. Ice particles are nucleated out from liquid water droplets. The nucleation mechanism can be considered stochastic immersion ice nucleation or contact ice nucleation, as long as they provide a uniform and constant volume ice nucleation rate in the mixed phase cloud region. The volume ice nucleation rate represents the number of ice particles generated per unit volume per unit time [148, 149]. Nucleated ice particles are assumed to be spherical and can grow or evaporate depending on the saturation ratio with respect to ice. The capacitance for the ice particle-mass relationship, the mass-maximum particle dimension relationship and the maximum particle dimension-terminal velocity relationship are the same as Equations A9-A13 in [91]. In these formulations, ice particles are treated as low density spheres, for which the capacitance-mass relationship is close to that derived by [138] based on measurements by [124] for hexagonal plates forming broad branches after 10 minutes of growth at saturation with respect to liquid water at $-12{ }^{\circ} \mathrm{C}$. The terminal velocity-maximum particle dimension is similar to that for low-density spheres given by [4]. 
Ice particles are seeded in the liquid cloud layer between $600 \mathrm{~m}$ and $800 \mathrm{~m}$ with 50 $m$ horizontal separation and $10 m$ vertical separation. For each seeding location, one ice particle with $10 \mu m$ initial radius is nucleated out every 8 seconds. Its growth and motion, including sedimentation, are calculated within the time dependent field. Trajectory for each ice particle is calculated with the Adams-Bashforth 2nd order method until it totally sublimates or falls to the ground (further details by [149]). The Lagrangian tracks do not include a contribution from LES subgrid-scale velocity fluctuations and this has two justifications. First, the problem studied here is focused on the large-scale motion of individual ice crystals. Single-particle dispersion statistics are reasonably well captured by LES because errors in Lagrangian velocity correlation time and velocity fluctuation magnitudes tend to offset each other [153]. This is in contrast to pair or multi-particle dispersion, for which sub-grid-scale velocity fluctuations are important when the initial particle separations are less than the grid scale [135]. Second, strongly sedimenting particles tend to smooth over small-scale, high-frequency velocity fluctuations within the turbulent flow [16]. The unresolved small scales in the LES are likely to be smoothed over based on the following observations. Throughout most of the computational domain, the turbulent kinetic energy (TKE) resolved by the LES is almost two orders of magnitude larger than the unresolved TKE. Specifically, except in the lowest 100 $\mathrm{m}$, the mean unresolved values are $0.005 \mathrm{~m}^{2} \mathrm{~s}^{-2}$ whereas the in-cloud resolved values are on the order of $0.4 \mathrm{~m}^{2} \mathrm{~s}^{-2}$. Thus, the subgrid velocity scale is about $0.06 \mathrm{~ms}^{-1}$, 
compared to the resolved velocity scale of $0.5 \mathrm{~ms}^{-1}$. The fall speed of ice particles exceeds $0.1 \mathrm{~ms}^{-1}$ for radii greater than $40 \mu \mathrm{m}$, which is achieved in a matter of minutes after the particles first appear (e.g., see Figure S1 in [148]). Therefore, in most of the simulated time the ice crystal fall speeds are significantly larger than the expected velocity fluctuations in the unresolved field.

In summary, 81920 ice particles with $10 \mu m$ initial radii are seeded uniformly within the liquid-cloud layer between $600 \mathrm{~m}$ and $800 \mathrm{~m}$ every $8 \mathrm{~s}$, with $50 \mathrm{~m}$ horizontal separation and $10 \mathrm{~m}$ vertical separation. It should be mentioned that by cloud in this work we refer to the layer where both liquid and ice are present, and "seed" here refers to ice initiation in the cloud, not related to artificial cloud seeding. The growth and trajecotry of each ice particle are calculated at one-second intervals within the time-dependent field from a LES cloud model. The position and radius of each ice particle, together with the air velocity at that position output every $8 \mathrm{~s}$ for further analysis. As the ice number concentration is very low, we ignored the dynamic and thermodynamic feedbacks of ice particles to the field, which is called the one way coupling. 


\section{Long Lifetime Ice Particles In A 3D Time Depen- dent Velocity Field}

For a constant crystal nucleation rate as we discussed in previous section (one every 8 seconds at each seeding location), we expected that the total number of ice particles in the mixed phase cloud would reach a steady state after a relatively short time: either on the order of one large eddy turnover time if dominated by fluid transport, or in the time required for a crystal nucleated at the top of the cloud to settle out in still air. The LES field has a turbulent boundary layer depth of $H \approx 800 \mathrm{~m}$ with a vertical velocity scale of $w^{\prime} \approx 0.45 \mathrm{~ms}^{-1}$, so that a large eddy time can be estimated as $H / w^{\prime} \approx 30$ minutes. The longest time needed for an ice particle to fall out of the $200 \mathrm{~m}$ thick mixed phase cloud in a still environment is found to be approximately $24 \mathrm{~min}$. This is calculated with the same method used to obtain the maximum ice particle diameter in section 1. As a first estimate, it would seem reasonable to assume that on time scales of half an hour, the total number of ice particles in the cloud should be almost constant due to a balance between formation and sedimentation. However, the total number of ice particles in the cloud increases monotonically over the full 1.5 simulated hours (not shown here). To understand this surprisingly long-term response we alter the perspective slightly and consider the question: if we seed ice particles uniformly within the cloud only at the beginning, 
how does the fraction of those ice particles in the mixed phase cloud decay with time? Results show that $2.8 \%$ of those ice particles are still in the cloud after 1.5 hours (black line in Figure 6.1 a, and surprisingly, 14.5\% still survive in the whole simulation domain. The fraction of surviving ice particles is much larger than $2.8 \%$, because ice particles still exist in the sub-cloud region (below the base of mixed phase cloud) after 1.5 hours and may still reenter the mixed phase cloud. As no other ice formation mechanism exists in the model, this means $14.5 \%$ of ice particles have lifetime longer than 1.5 hours. Considering the time scales discussed previously, the existence of such a large fraction of long-lifetime ice particles was a surprise.

Ice particles might have long lifetimes if they are suspended within the cloud region in a steady updraft [148], or if they fall out of the cloud in downdraft regions and get back into the cloud several times [30]. To find which mechanism is more important, we define the recycling number $(R N)$ as the number of times an ice particle reenters the liquid cloud layer (above $600 \mathrm{~m}$ in this study). The probability density functions (PDF) of ice particle lifetime for different $R N$ are shown in Figure 6.1 b. We also define the factor $\chi$ as the fraction of ice particles surviving after time $\tau$,

$$
\chi(\tau)=1-\int_{0}^{\tau} P D F(t) d t .
$$



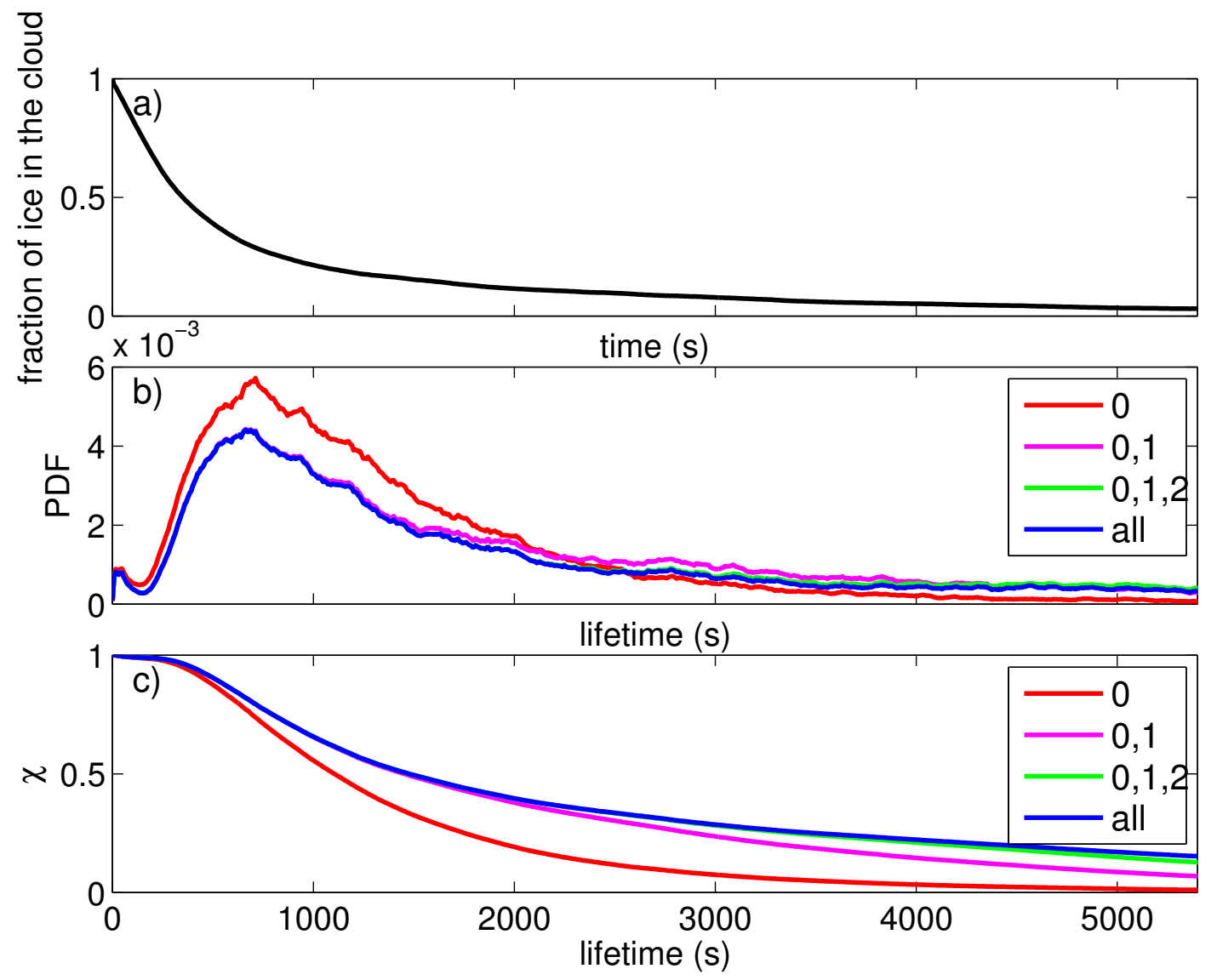

Figure 6.1: (a) Fraction of ice particles remaining in the cloud. The black line is from the LES time dependent field. (b) PDF of ice particle lifetimes. Different colors represent different recycling numbers. (c) Ice particle survival fraction after a specific lifetime $\tau$, defined as $\chi(\tau)=1-\int_{0}^{\tau} P D F(t) d t$.

Results are shown in Figure 6.1 c. It is clear that the fraction of recycling particles make the largest contribution to the surviving particles as time progresses, and nearly all of the surviving ice particles have been recycled at least once after $4000 \mathrm{~s}$.

Figure 6.2 shows the time evolution of the normalized size distribution of ice particles in the mixed phase cloud. Again, this result is for ice particles generated only at 


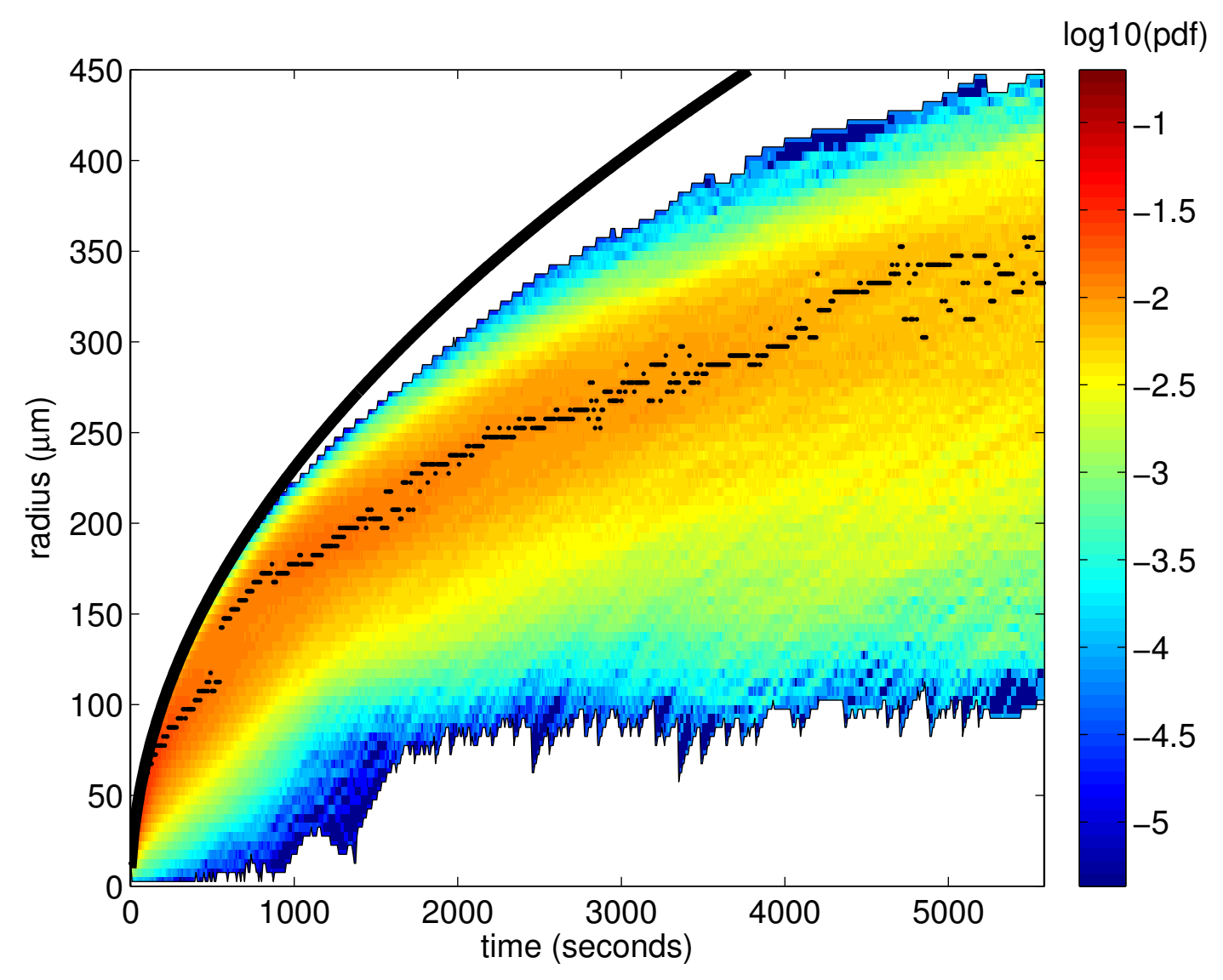

Figure 6.2: Time evolution of the probability density function for ice particle radius in the mixed phase cloud. The black curve is the growth of an ice particle under a constant supersaturation, which is set to be the initial supersaturation in the mixed phase cloud. The block dots indicate the maximum value of the PDF at each time.

$t=0$. Black dots in the figure represent the radii with the highest probability at different time. The black thick line represents the growth curve of an ice particle under a constant supersaturation equal to the initial supersaturation in the mixed phase cloud. It shows that the upper boundary of the PDF and the maximum probability points almost follow this growth curve at the beginning, while after approximately $1000 s$ they deviate substantially. The reason is that the ice supersaturation ratio 
is almost time independent in the mixed phase cloud due to the existence of liquid droplets and steady cloud temperature. When ice particles remain within the mixed phase cloud region, they can grow under the similar supersaturation with respect to ice. But after some time most of them will fall into a region of subsaturation with respect to ice. Although some of them might be recycled into the mixed-phase region again, the average growth rate is smaller due to the time spent below the liquid cloud base. In particular, it is interesting to see that there exists a lower limit radius (about $100 \mu \mathrm{m}$ ) after $2000 \mathrm{~s}$. This lower limit is presumably a result of the subcloud region still being supersaturated with respect to ice, and recycled ice crystals therefore grow even as they are below the mixed phase region. At the same time, some subset of lucky ice particles will exist in the supersaturated region for a long time, thus they can always grow, leading to a broad distribution.

Figure 6.3 displays trajectories for ten long-lifetime ice particles in the mixed phase cloud. These in-cloud trajectories are plotted in radius-time coordinates with the color representing the recycling number. For clarity in tracing each trajectory the time gap when an ice particle is below the mixed-phase cloud is marked with a black dashed line. The gray background shading shows the outlines of the size PDF from Figure 6.2 for comparison. The large star symbols represent average ice particle radius in the whole mixed phase cloud region, and the color represents the average recycling number at that time. Results show that on average, ice particle radius 


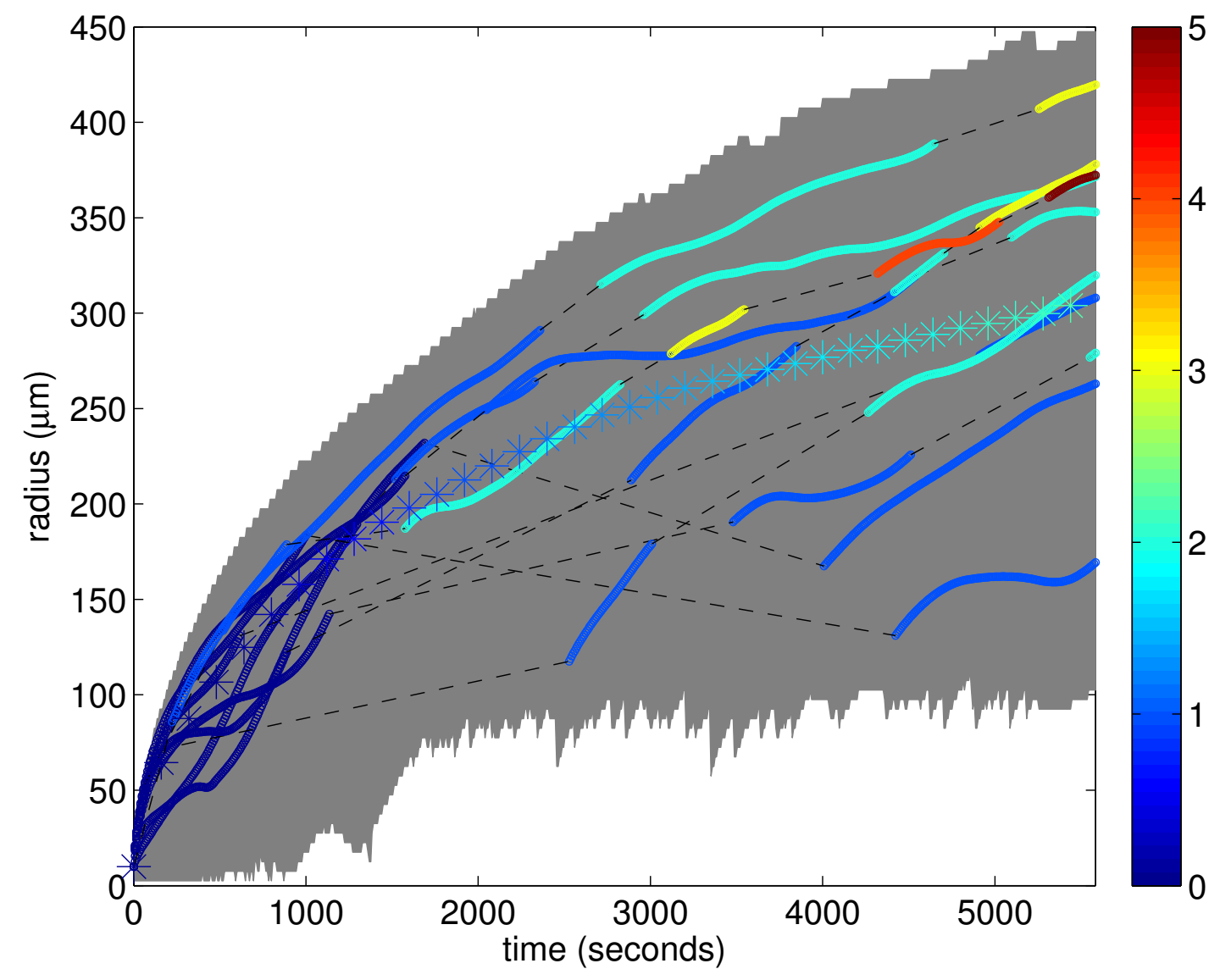

Figure 6.3: Radius versus time for ten long-lifetime ice particles in the mixed phase cloud. The ice particle radius is plotted only for the times when it is in the mixed phase cloud, with dashed lines joining the regions between which the ice particles are below liquid cloud base. Color here represents the recycling number. The large star symbols represent the average ice particle radius over the whole mixed phase cloud region, and its color represents the average recycling number at that time. The grey shading indicate the full range of ice particle size distribution at each time as shown in Figure 6.2.

and recycling number both increase with time. Especially revealing to note that the average recycling number is close to the ratio of time $t$ to the large eddy turn over time $\left(\tau_{l} \approx 30 \mathrm{~min}\right.$ in this case). It suggests that the recycling process has a connection to the eddy structure. 


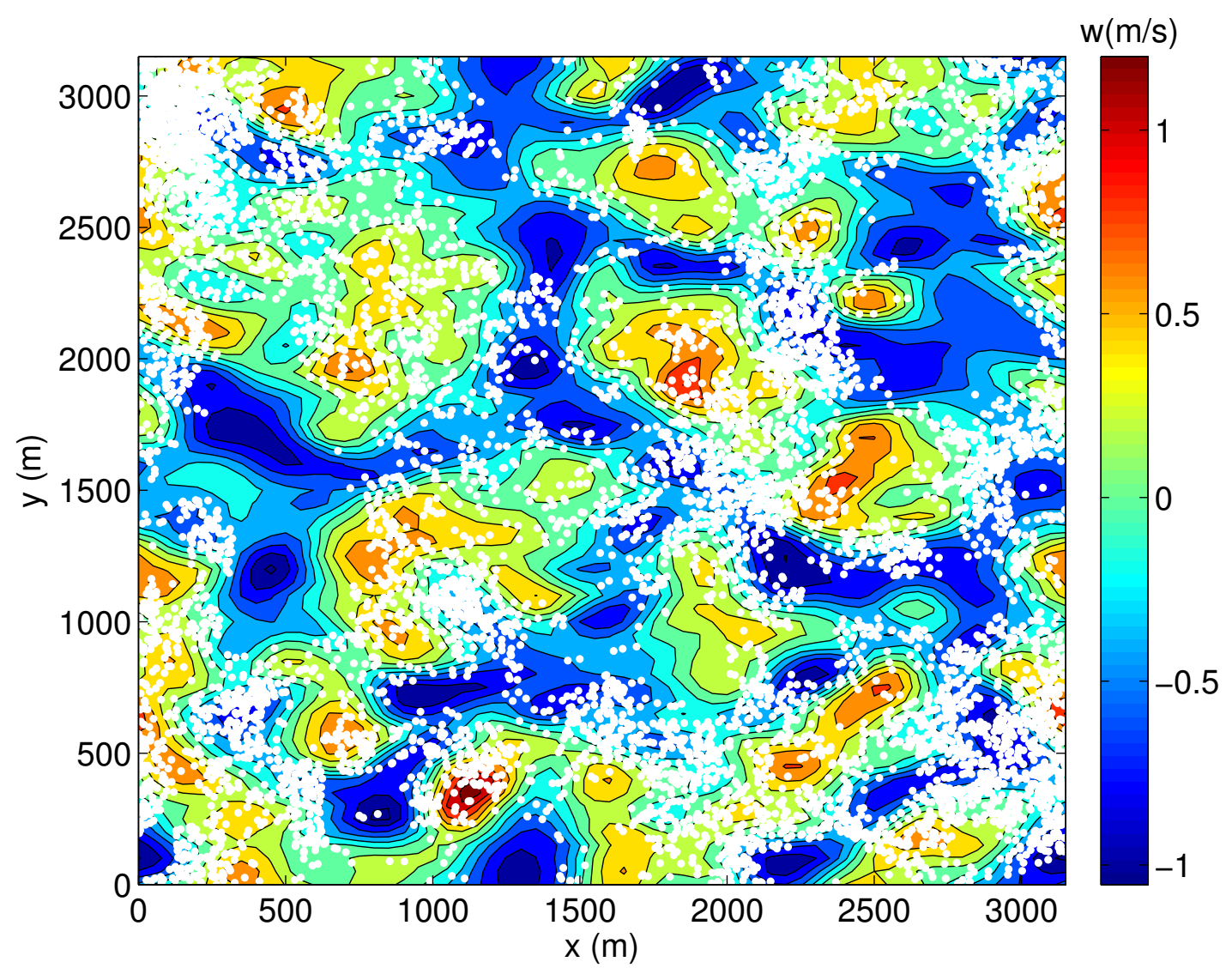

Figure 6.4: Initial horizontal seeding location for long lifetime ice particles. Background color represents the initial air vertical velocity.

We also are interested in the initial seeding location of long lifetime ice particles (particles still surviving after 1.5 hours). To that end we ask a simple question: are the initial seeding locations for long-lifetime ice particles uniformly distributed in the domain or clustered? Figure 6.4 shows the initial horizontal seeding location for the ice particles still surviving after 1.5 hours. The background color is the mean air vertical velocity in the mixed phase cloud region at the time of ice particle seeding. It can be seen that the long lifetime ice particles are strongly clustered within the cloud. It is also apparent that clustering tends to be more correlated with updrafts 
than downdrafts in Figure 6.4. Statistical analysis shows that $17 \%$ of particles initially seeded in updraft regions have lifetime longer than 1.5 hours, while only $13 \%$ of particles initially seeded in downdraft regions have such long lifetimes. The correlation is therefore positive, but rather modest. It is perhaps not a surprise that long lifetime ice particles are not obviously linked with the initial vertical velocity region. Because the velocity field is time dependent, an updraft region in the initial field can quickly transform to a downdraft region, on average with a time scale on the order of the large-eddy correlation time, but in any specific realization such as that chosen here, the individual eddy lifetimes are randomly distributed about the expected time. Therefore, ice particles initially seeded in an updraft velocity do not always have long lifetime, or conversely, ice particles initially seeded in a downdraft region do not always have short lifetime. The positive correlation means that there is only a weak tendency for ice particles to be more likely to have long lifetime if they are initially seeded in the updraft region than downdraft region. To further study the cluster behaviour of initial horizontal seeding locations, we convert the dots in Figure 6.4 to a 2D density field and calculate the 2D autocorrelation functions for the vertical velocity field and density field. As is clear from simple inspection, even if not strongly cross-correlated, the fields have similar correlation lengths: the integral length for the particle density field is $180 \mathrm{~m}$, and that for the vertical velocity field is $142 \mathrm{~m}$. This is strong indication that there is a connection between the cluster structure of long-lifetime ice crystal and the large-eddy structure of the turbulent 
flow in which the particles are embedded. Because most of these long lifetime ice particles have been recycled at least once(See Figure 6.1), we can also expect that the mechanism for the recycling process is linked to the spatial large-eddy structure.

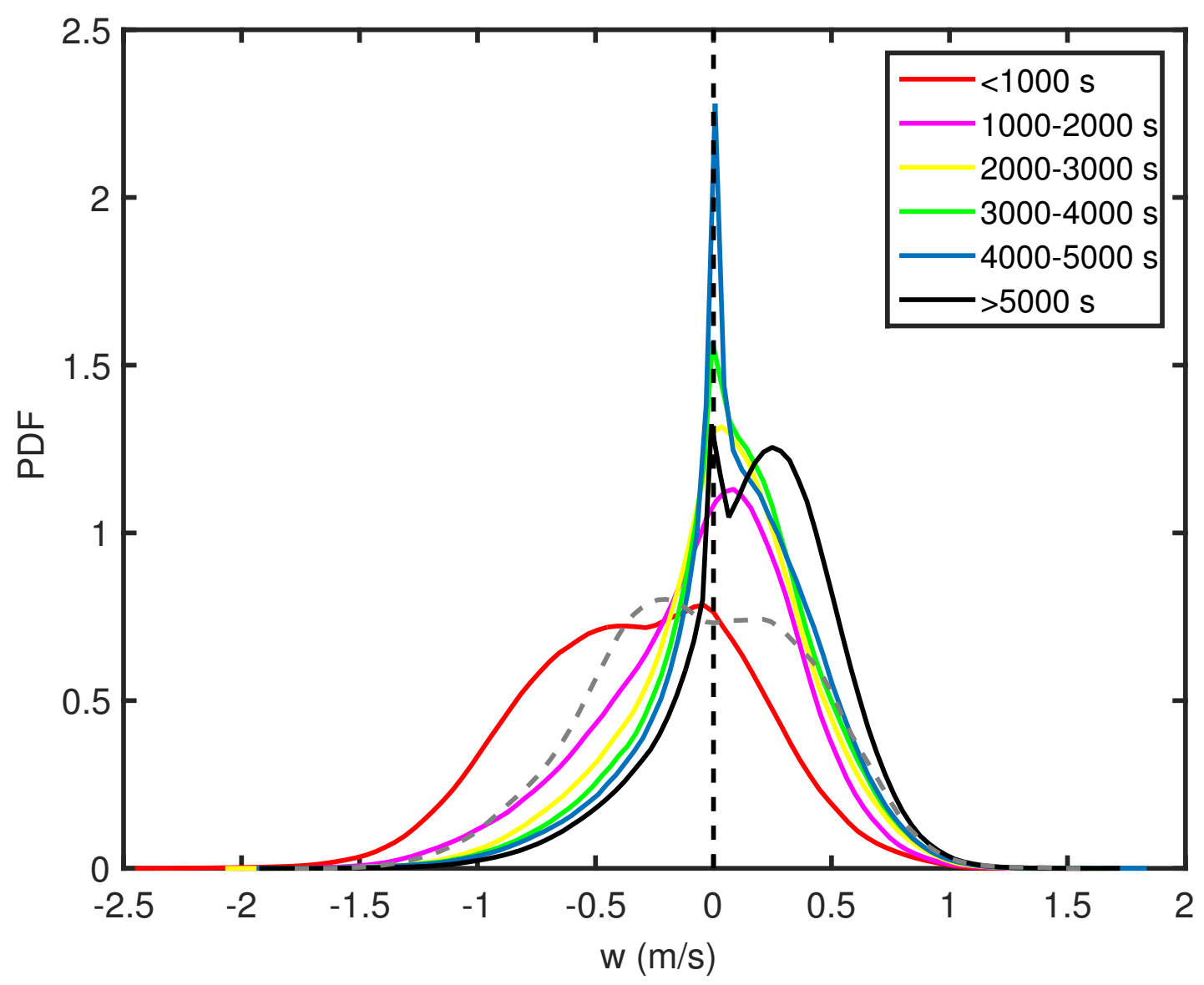

Figure 6.5: Probability density function of vertical air velocity conditioned on ice particle location, for ice particles with different ranges of lifetime. The gray dashed line is the initial vertical air velocity for all ice particles seeded in the cloud, which is centered on $w=0$.

The role of vertical velocity can be further investigated by considering conditional statistics based on ice particle locations. Figure 6.5 shows the probability density 
function of vertical air velocity for ice particles with different lifetimes. It can be seen that short lifetime ice particles have a higher probability of residing in downdraft regions, while long lifetime ice particles display a higher probability of residing in updraft regions. For comparison's sake, the PDF of the vertical velocity for the initial seeding locations is centered on zero (gray dashed line). More interestingly, for ice particle lifetimes greater than $5000 \mathrm{~s}$, a peak at about $0.4 \mathrm{~ms}^{-1}$ emerges, which likely corresponds to a stationary growth region in which updraft velocity is roughly equal in magnitude to the ice particle terminal speed. This is an indication of the quasi-steady growth mechanism of [148].

In summary, long-lifetime, recycling ice particles exist in mixed phase clouds, and are present in such numbers that they can be of consequence to the ice water content and the maximum crystal size, which can affect radiation balance and in-cloud heterogeneous chemical reaction. Understanding the dynamic and microphysical coupling that leads to this observation is challenging for complex, three-dimensional trajectories in a time dependent LES field. We address this in a more idealized way in the following section. 


\section{Long Lifetime Ice Particles In A 2D Idealized Ve- locity Field}

To investigate the motion of ice particles within the cloud-topped boundary layer

we take the simplified kinematic approach of [75] as inspiration and use a prescribed 2D cellular velocity field. This type of approach has proven useful for testing microphysical parameterizations $[79,114]$. Here we apply it to explore the mechanisms for existence of long-lifetime ice particles. Specifically, based on the 3D time-dependent LES study in the previous section, we understand that the ice crystal recycling is a result of the interplay between the large-scale structure of the velocity field and the growth and associated fall speed of the ice crystals carried within that velocity field. Trajectories simulated within a 2D, time-independent velocity field are highly idealized, but they clearly represent the interactions between particle settling, particle growth and evaporation, and the large-eddy structure within a cloud-topped boundary layer. Connections between the idealized 2D results presented in this section and the more realistic 3D results from the previous section will be further discussed in the concluding section.

The horizontal and vertical velocities for the 2D kinematic field are given by 


$$
\left\{\begin{array}{l}
u=u_{0} \cos \left(\frac{2 \pi x}{L}\right) \sin \left(\frac{2 \pi z}{H}\right) \\
w=w_{0} \sin \left(\frac{2 \pi x}{L}\right) \sin \left(\frac{\pi z}{H}\right)
\end{array}\right.
$$

where $u_{0}$ and $w_{0}$ are horizontal and vertical velocity amplitudes, and $L$ and $H$ are horizontal and vertical domain ranges, respectively. For simplicity, we assume the horizontal boundary is periodic, and $u_{0}=w_{0}=v_{\max }$, where $v_{\max }$ is a maximum velocity parameter. The thermodynamic profiles (pressure, temperature, supersaturation ratio) representative of a well-mixed cloud-topped boundary layer are adapted from a previous study (Figure 5.1). Figure 6.6 shows the flow structure with $L=3150 \mathrm{~m}, H=800 \mathrm{~m}$ and $v_{\max }=2 \mathrm{~ms}^{-1}$, and the grey shaded region represents the liquid cloud. It should be mentioned that although this idealized field is time independent, we can still use it to study the dynamic and microphysical coupling effects because the largest eddies are a basic structure of a turbulent boundary layer and have lifetimes long compared to the time for a large ice particle to fall through the cloud layer in still air (see previous section).

Ice particles are seeded uniformly in the liquid cloud layer between $600 \mathrm{~m}$ and $800 \mathrm{~m}$ with $50 \mathrm{~m}$ horizontal separation and $10 \mathrm{~m}$ vertical separation. The initial ice particle radius is $10 \mu \mathrm{m}$. Trajectories in the $2 \mathrm{D}$ field are calculated using an Adams-Bashforth second order method, accounting for ice crystal growth/evaporation and sedimentation. The total simulation time is 1.5 hours. Six different velocity 


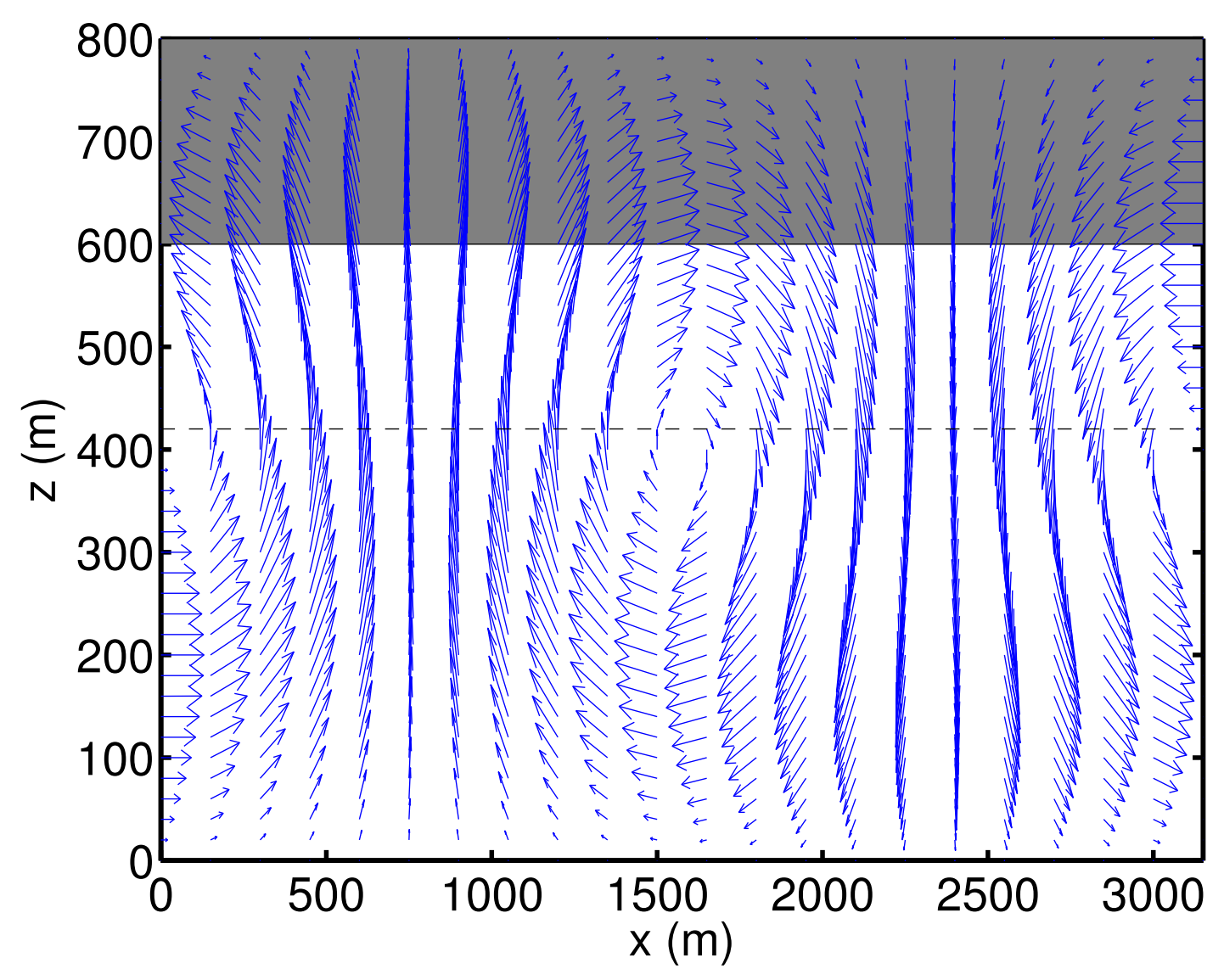

Figure 6.6: Velocity field in the idealized 2D field with $v_{\max }=2 \mathrm{~ms}^{-1}$. The arrows indicate the wind direction and magnitude. The black dashed line (at $420 \mathrm{~m}$ ) represents the level above which the environment is supersaturated with respect to ice, below is the region of subsaturation with respect to ice, and the shaded area denotes the mixed-phase region.

amplitudes are tested: $v_{\max }=0.1,0.25,0.5,1.0,2.0,4.0 \mathrm{~ms}^{-1}$. The $\chi$ versus lifetime with different $v_{\max }$ are shown in Figure 6.7. Red lines represent non-recycling ice particles, and blue lines represent all ice particles. The results show that for $v_{\max }$ larger than $1 \mathrm{~ms}^{-1}$, recycling ice particles exist in the mixed phase cloud. Increasing $v_{\max }$ decreases $\chi$ for non-recycling ice particles, but increases $\chi$ for recycling ice particles for a specific lifetime (Figure 6.7 d-f). The reason for this is that a larger 
air velocity transports ice particles out of the mixed phase cloud in the downdraft region quickly, thus decreasing the fraction of non-recycling ice particles. At the same time, ice particles might quickly reenter in the updraft region and go back into the cloud again before they totally evaporate, thus increasing the fraction of recycling ice particles. It's interesting to see that the non-recycling ice particles also have long lifetime for $v_{\max }=4 \mathrm{~ms}^{-1}$. This is because more ice particles can be held in the quasi-steady state region (where updraft velocity is larger than ice terminal velocity) for a longer time compared with $v_{\max }=2 \mathrm{~ms}^{-1}$.

However for smaller $v_{\max }\left(0.1,0.25,0.5 \mathrm{~ms}^{-1}\right)$ the blue lines coincide with the red ones, which means that there are no recycling ice particles in the cloud during the 1.5 hours (Figure $6.7 \mathrm{a}-\mathrm{c})$. In addition, when increasing $v_{\max }, \chi$ does not decrease monotonically like in the larger $v_{\max }$ cases. The reason is that for the smallest $v_{\max }=0.1 \mathrm{~ms}^{-1}$, ice particles can't be suspended in either the mixed phase cloud or in the subcloud region. So the longest lifetime of ice particles is less than 5000 seconds. For $v_{\max }=0.25 \mathrm{~ms}^{-1}$, ice particles can't be suspended in the mixed phase cloud, but they can be trapped in the subcloud region where the air velocity is larger (Note that the maximum vertical velocity is at $400 \mathrm{~m}$ ). Although there are no recycling ice particles for $v_{\max }=0.25 \mathrm{~ms}^{-1}$, there exist long lifetime ice particles. Those ice particles trapped in the subcloud region could have longer lifetime, but are not considered recycling ice particles by our definition, which requires that ice 

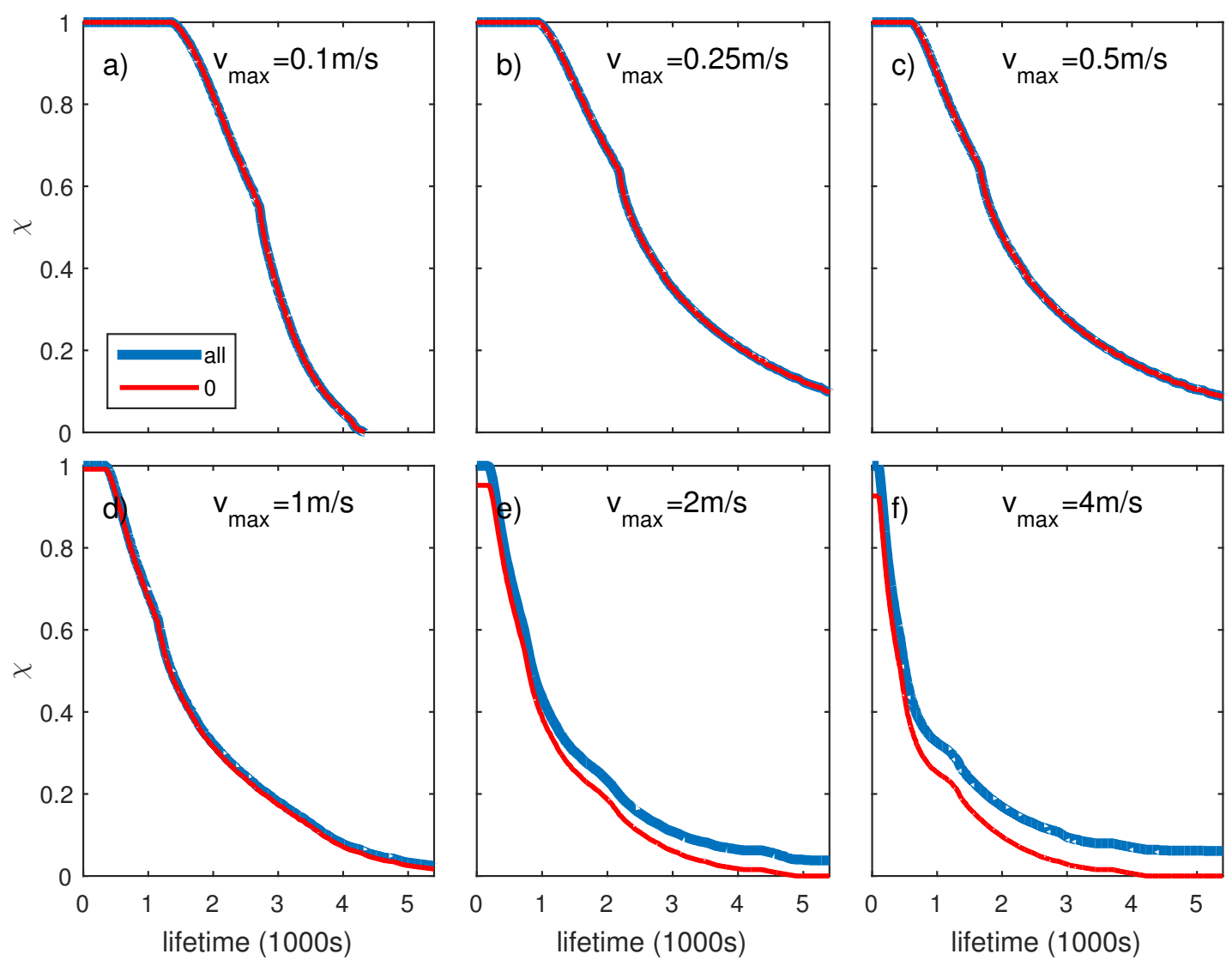

Figure 6.7: $\chi$ versus lifetime in 2D field with different maximum velocity $v_{\max }$. Here the thick blue line (all) includes both recycling and non-recycling ice particles, and the thin red line (0) only includes non-recycling ice particles.

particles reenter the mixed phase cloud (above $600 \mathrm{~m}$ ). Why ice particles can be trapped in the subcloud region will be discussed later. The kink observed in Figure 6.7 a-e are probably a result of two different ice particle removal rates: before the kink the removal is dominated by non-trapped particles, whereas after the kink it is dominated by trapped particles. By trapped, we mean ice particles that have recirculation and long lifetimes, even if the recirculation does not take them back into the mixed-phase cloud, such as in Figure 6.7 a-c (i.e., trapped particles are not 
necessarily classified as recycling particles).
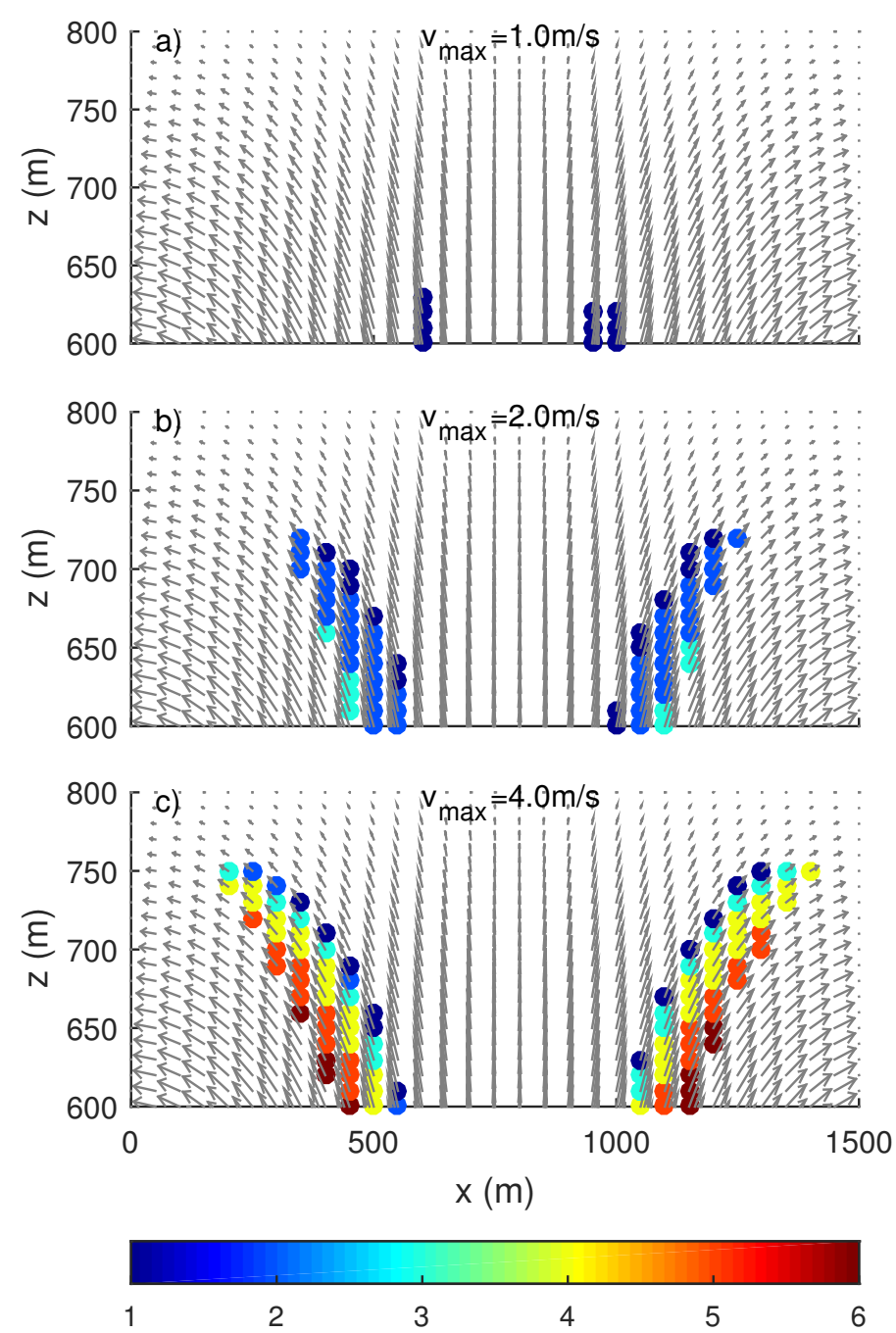

Figure 6.8: Locations of recycled particles when first seeded for three different $v_{\max }$ : a) $1.0 \mathrm{~ms}^{-1}$, b) $2.0 \mathrm{~ms}^{-1}$, c) $4.0 \mathrm{~ms}^{-1}$. Color represents the recycling number. It should be mentioned that the z-axis is restricted to the mixed-phase layer and the $\mathrm{x}$-axis is limited to the upward branch of the circulation in Figure 6.6.

Figure 6.8 shows the initial seeding locations of recycling ice particles for $v_{\max }=1.0$,

2.0, and $4.0 \mathrm{~ms}^{-1}$. Colors of the points in each figure represent $R N$ at the end 
of the simulation (after 1.5 hours). It can be seen that for any given case, taking $v_{\max }=4.0 \mathrm{~ms}^{-1}$ as an example, the initial seeding locations of recycling ice particles occupy a distinctive, well defined region of the cloud, symmetric about the center of the updraft velocity region. Due to the symmetry of the vortex structure this can be considered the vicinity of a stagnation point. The recycling number is larger when the seeding location is lower in height (for a given $x$ ), or close to the center of the vortex (bottom left and right corners in each subplot of Figure 6.8). In particular, we note that the recycling number is discontinuous from the center of the updraft region to the vortex center. This discontinuity implies that there should be a sharply defined seeding region for recycling ice particles. Furthermore, increasing $v_{\max }$ increases both the seeding location range and the ice particle $R N$.

To better understand the interactions between dynamic and microphysical effect on long lifetime ice particles, three different trajectories for the $v_{\max }=2.0 \mathrm{~ms}^{-1}$ case are analyzed. The trajectory for an ice particle seeded at $x=800 \mathrm{~m}$ and $z=790$ $\mathrm{m}$ is shown in Figure 6.9 $a$. Ice particle is seeded very close to the center of the updraft region $(787.5 \mathrm{~m})$ where the horizontal velocity is very small. So it can grow and be suspended in the cloud for a long time before it falls out of the liquid cloud region. Updraft and very small horizontal velocity define a 'quasi-steady' growth region where ice particles cannot fall out until they grow larger, in agreement with the conceptual picture of [148]. In the quasi-steady region, an ice particle grows 


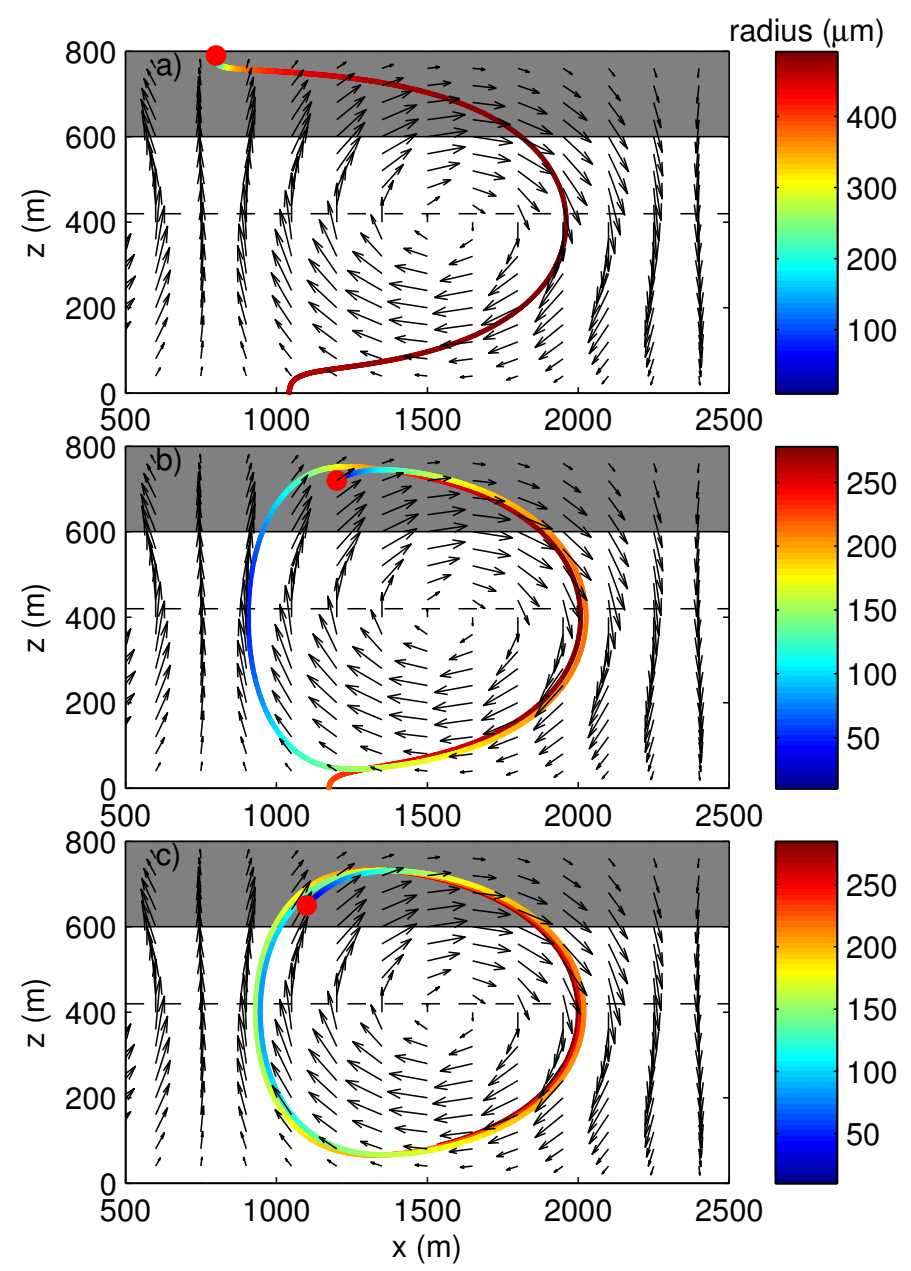

Figure 6.9: Trajectories of three different ice particles in an analytical velocity field with $v_{\max }=2.0 \mathrm{~ms}^{-1}$ (see Equation 6.2 for explanation). Red dots represent initial seeding locations (x,z) at (a) $(800 \mathrm{~m}, 790 \mathrm{~m})$, (b) $(1200$ $\mathrm{m}, 720 \mathrm{~m})$, and $(\mathrm{c})(1100 \mathrm{~m}, 650 \mathrm{~m})$. The color bar here represents the radius of the ice particle. Subcloud region is below $600 \mathrm{~m}$ and subsaturated region with respect to ice is below $420 \mathrm{~m}$, illustrated in Figure 6.6.

rapidly because of the high supersaturation over ice in the mixed phase cloud. Although the horizontal velocity is very small, the ice particle will eventually be transported to the downdraft region after a long time, where the ice particle will fall quickly without recycling. The lifetime of the ice particle in Figure 6.9 $a$ is 4848 
s, and we define this type of long lifetime ice particles as Type 1: quasi-steady growth.

The trajectory for an ice particle seeded at $x=1200 \mathrm{~m}$ and $z=720 \mathrm{~m}$, which has a lifetime of $4524 \mathrm{~s}$, is shown in Figure $6.9 \mathrm{~b}$. The ice particle is recycled once before it falls to the ground. We define this type of long lifetime ice particle as Type 2: recycled. In this case, the ice particle grows in the cloud region at first, but is quickly transported to the downdraft region and falls out of the cloud. Below $400 \mathrm{~m}$ where water vapor is undersaturated with respect to ice, the ice particle sublimates. However, the particle is sufficiently large to survive in this sublimation region, and eventually to be transported to the updraft region within the converging air motion. Particles have a chance to reenter the cloud, as long as they do not fall to the ground or evaporate completely. Once an ice particle is recycled to the cloud, it can grow in the water saturated region again, transport to the downdraft region quickly, and so forth. Eventually the ice particle will fall to the ground when it is large enough that its fall speed does not allow it to be swept back into the updraft region again. An example of an extreme case is for an ice particle seeded at $x=1100 \mathrm{~m}$ and $z=650$ m: the crystal still exists at the end of the simulation time (Figure 6.9 c). Therefore its lifetime is longer than $5400 s$ (1.5 hours). It is worth noting that the seeding region that produces recycling ice crystals has very sharp boundaries and that relatively small changes in seeding location change the number of recycling events. We expect that this is a result of the idealized, two-dimensional, time-independent 
flow, and that such organization is unlikely in a three-dimensional, time-evolving flow. The location of seeding events in regions that evolve into stagnation points at the top of the cloud is probably more general.

Compared with Type 1, quasi-steady growth trajectories, Type 2, recycled trajectories for long lifetime ice particles have several interesting properties. First, as an ice crystal grows, the recycling trajectory or orbit in the updraft region will shift to the larger updraft velocity side compared with the previous one, while in the downdraft region, the new trajectory will shift to the smaller downdraft velocity side. In addition, the radius of curvature of the trajectory in the updraft region is larger than that in the downdraft region. All these properties are due to the gravitational settling of ice particles: gravity always drags ice particles away from the air streamlines.

An alternative way to look at the long lifetime ice particles is in a radius-height plot Figure 6.10, where color represents vertical velocity of the air. For Type 1 trajectories, the ice particle grows quickly in the updraft region in a quasi-steady balance between the updraft and the fall speed, until it finally moves quickly to the downdraft region and falls out of the cloud. For Type 2 trajectories, although the size of the ice particle increases in the supersaturated region (e.g., cloud region) 


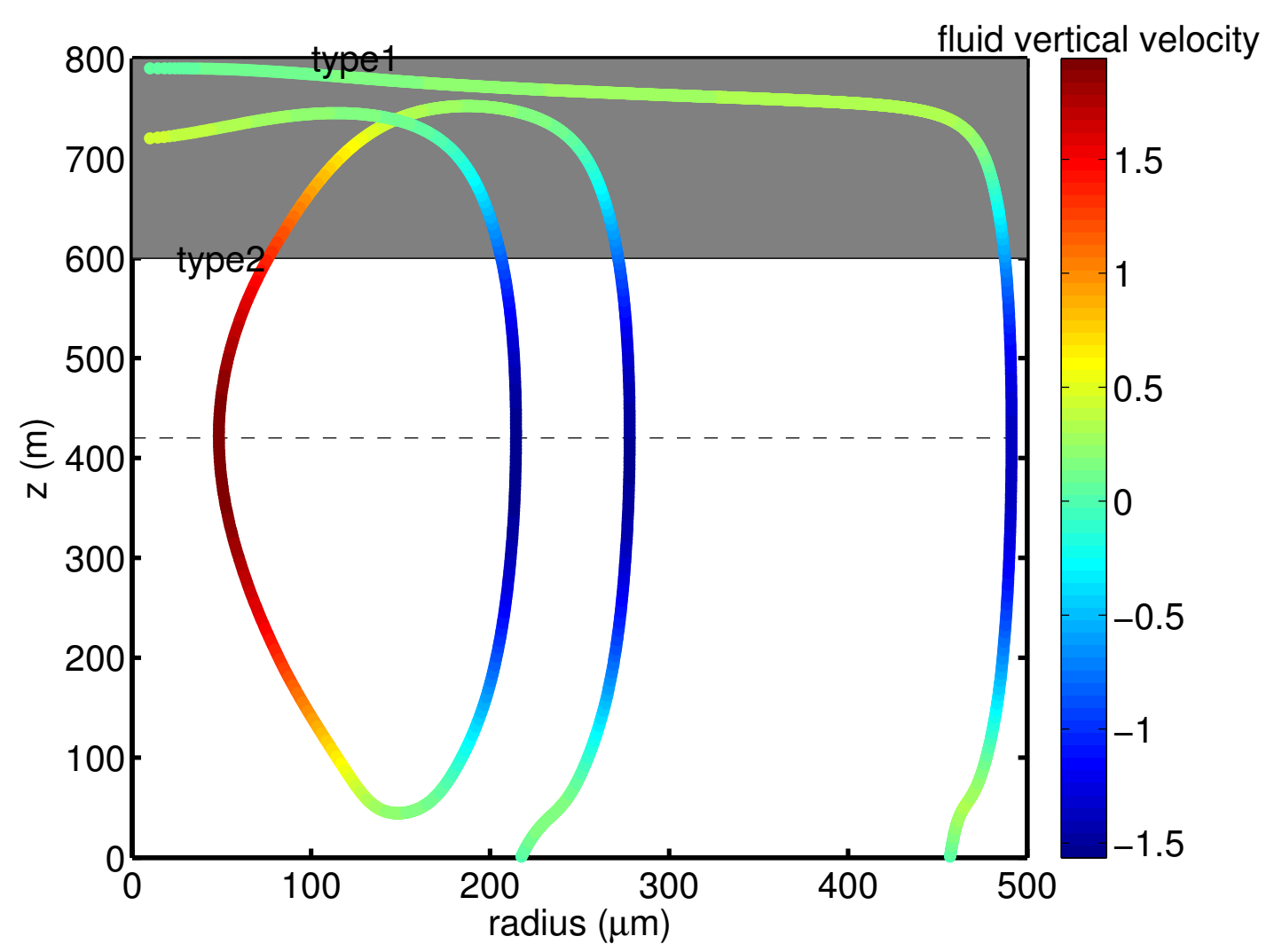

Figure 6.10: Height versus radius for two types of long lifetime ice particles with $v_{\max }=2.0 \mathrm{~ms}^{-1}$. Colors here represent the air vertical velocity. Type 1 is initially seeding at $(800 \mathrm{~m}, 790 \mathrm{~m})$ and Type 2 is seeded at $(1200 \mathrm{~m}, 720$ $\mathrm{m}$ ). Subcloud region (below $600 \mathrm{~m}$ ) and subsaturated region with respect to ice (below $420 \mathrm{~m}$ ) are the same as in Figure 6.6.

and decreases in the subsaturated region (e.g., subcloud region), on average the ice particle size increases after each cycle. This is because the center of the orbit is sufficiently close to the level of ice saturation. In the end, the ice particle falls to the ground when it is large enough that it can't be recycled again. The radius-height plot eliminates the complexity of the full trajectory, so we consider using it for visualizing long-lifetime crystal growth trajectories in a fully $3 \mathrm{D}$ velocity field. We note in passing that a similar visualization approach has been used by [60], albeit 
with a different intended purpose. It should be mentioned that it is not necessary to have looping trajectories within the phase space: Ice particles can continuously grow if the trapping region is always supersaturated with respect to ice. Figure 6.11 shows radius-height plots for four selected ice particles in the 3D LES. Trajectory 1 shown in Figure 6.11 is for an ice particle initially seeded at $(3050 \mathrm{~m}, 150 \mathrm{~m}, 615$ m) in Cartesian coordinates. This particle totally evaporates in the sub-cloud region after $3088 \mathrm{~s}$ without recycling, which is close to Type 1. Trajectory 2 in Figure 6.11 is for an ice particle seeded at (3000 m, $2600 \mathrm{~m}, 605 \mathrm{~m})$. The ice particle is recycled several times and finally falls to the ground after $5400 \mathrm{~s}$. Trajectories 3 and 4 in Figure 6.11 show examples of ice particles with lifetimes longer than $5400 \mathrm{~s}$. All trajectories 2-4 belong to Type 2 . It can be seen that the long lifetime ice particles neither fall out directly to the ground, nor evaporate quickly in the subcloud region. Instead, they were trapped in the vortex-like field. If the trapping region is mostly supersaturated with respect to ice, the ice particles will grow, e.g. trajectory 4 . In contrast, if the trapping region is subsaturated, the ice particle will evaporate, e.g. trajectories 1, 2 and 3 in the subcloud region. But some trajectories (e.g., 2 and 3) do not show rapid evaporation because the region just below the mixed-phase cloud base is still supersaturated with respect to ice (Figure 5.1). In addition, when the environment can reach ice supersaturation even in the sub-liquid-cloud region, ice particles can be trapped in these regions until they grow large enough that the updraft cannot hold them, e.g., trajectory 2 . 

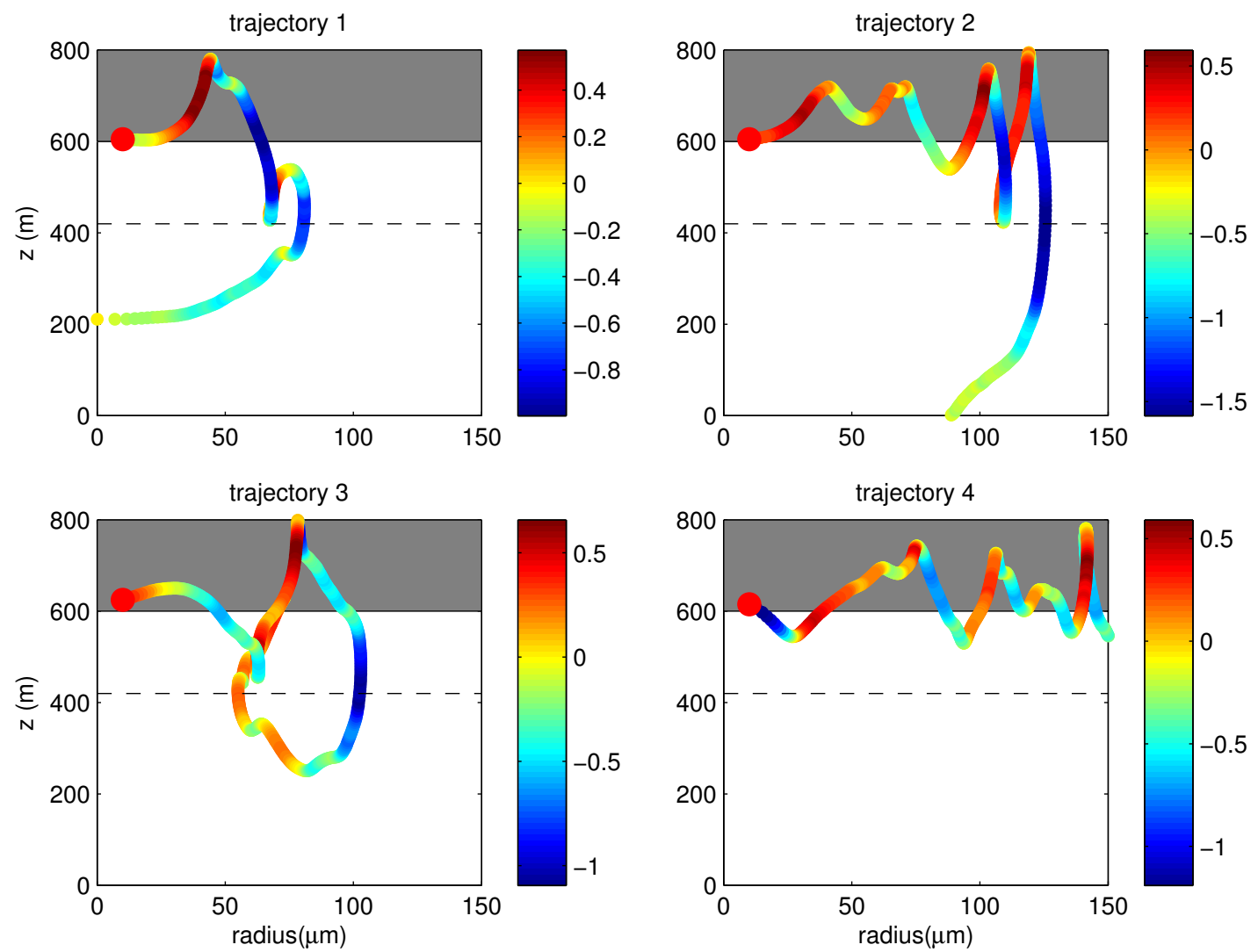

Figure 6.11: Height versus radius for four specific ice particles in the 3D time dependent field. Grey shading indicate the mixed phase cloud region and the colorbar represents the vertical velocity (in $m s^{-1}$ ). Red dots represent the initial seeding locations.

In summary, moderate eddy strengths can suspend or trap ice particles in a mixed phase cloud. If ice particles are seeded close to the center of an updraft region stagnation points, where the horizontal velocity is very small, they can be suspended or levitated in the mixed phase cloud for a long time before they fall out. If ice particles are seeded in the region where horizontal velocity is not so small, they can be trapped in the eddy. However, not every ice particle can be trapped. For example, 
large ice particles can't be trapped in the eddy due to large terminal velocity. In addition, not all trapped ice particles can have long lifetime. They can't have long lifetime if they totally evaporate in the subcloud region (e.g. ice particles are seeding in the downdraft region). Results above also show that increasing air velocity will increase the number of recycling ice particles, their recycling number and therefore their maximum size. It should be mentioned that the definition of a recycled ice particle depends on the assumption of cloud base, while the definition of trapped ice particle depends on its trajectory only. Ice particles can't be recycled if the air velocity is very small (e.g. $v_{\max }=0.25 \mathrm{~ms}^{-1}$ ), in which case ice particles are trapped only in the sub cloud region and usually evaporate slowly. In general, the dynamic structure of the mixing layer affects the trapping region and the thermodynamic properties within the trapping region determine the growth of ice particles.

\section{Analytical Solutions For Trajectories Of Constant- Size Ice Particles In Simple Velocity Fields}

From the study of trajectories in an idealized 2D velocity field we see that recycling ice particles have very interesting trajectories that depend on seeding locations, so we take yet another step in idealization and consider analytical solutions for the 
trajectories of fixed-size particles. We can then use the analytical trajectories to ask the following questions: Can we find the regions in which ice particles can be trapped in the boundary layer? Can we estimate the size of ice particles that cannot be trapped for a specific eddy strength? This would allow us to estimate the maximum size ice crystal that can be generated in a turbulent mixed-phase cloud. To that end, we derive analytical solutions for constant-size-particle trajectories in two simple air vortex fields.

We use polar coordinates $(r, \theta)$, where $r$ is the distance from the origin and $\theta$ is clockwise angle. We assume the particle terminal speed is a constant $v_{t 0}$ in the downward direction (constant particle size). Therefore, the particle's velocity in polar coordinates is

$$
\left\{\begin{array}{l}
v_{p, r}=v_{a, r}+v_{t 0} \sin \theta \\
v_{p, \theta}=v_{a, \theta}+v_{t 0} \cos \theta
\end{array}\right.
$$

where the subscripts $p$ and $a$ represent particle velocity and air velocity, respectively, and the subscripts $r$ and $\theta$ represent radial and azimuthal directions, respectively. Because $v_{p, r}=d r / d t$ and $v_{p, \theta}=r d \theta / d t$, we can get the governing equation for the trajectory through the quotient $v_{p, \theta} / v_{p, r}$, 


$$
\frac{r d \theta}{d r}=\frac{v_{a, \theta}+v_{t 0} \cos \theta}{v_{a, r}+v_{t 0} \sin \theta} .
$$

If the radial air velocity is zero $v_{a, r}=0$, Equation 6.4 can be rewritten as,

$$
\frac{d f}{d r}+\frac{f}{r}=-\frac{v_{a, \theta}}{r v_{t 0}}
$$

where $f \equiv \cos \theta$. For a specific air velocity field it may be possible to obtain an analytical solution for a particle trajectory. We take as an idealized model for a large-scale boundary layer structure the Rankine vortex (combination of a solid-body core and an irrotational velocity field outside), which is often used for representing vortex structures in the atmosphere. We continue by obtaining analytical solutions for solid-body and irrotational vortices, with the understanding that the Rankine vortex can be constructed as a peicewise-continuous velocity field.

\section{Solid Body Rotation Field}

We assume the air velocity in polar coordinates is $v_{a, r}=0$ and $v_{a, \theta}=\omega r$, where $r$ is the distance from the origin and $\omega$ is a constant. This air field corresponds to solid 
body rotation with constant angular velocity $\omega$. Assuming the particle is initially located at $\left(r_{0}, \theta_{0}\right)$, its trajectory in polar coordinates can be solved analytically from Equation 6.5,

$$
f=-\frac{\omega_{0}}{2 v_{t 0}} r+\frac{f_{0} r_{0}+\frac{\omega_{0}}{2 v_{t 0}} r_{0}^{2}}{r},
$$

where $f_{0}=\cos \theta_{0}$. Because $0 \leq \theta \leq 2 \pi$ and $f=\cos \theta$, thus $-1 \leq f \leq 1$,

$$
-1 \leq-\frac{\omega_{0}}{2 v_{t 0}} r+\frac{f_{0} r_{0}+\frac{\omega_{0}}{2 v_{t 0}} r_{0}^{2}}{r} \leq 1 .
$$

Since $r>0$, the above inequality can be written as two simple inequalities,

$$
\left\{\begin{array}{l}
g_{1} \leq 0 \\
g_{2} \geq 0
\end{array}\right.
$$

where,

$$
\left\{\begin{array}{l}
g_{1}=\frac{\omega_{0}}{2 v_{t 0}} r^{2}-r-f_{0} r_{0}-\frac{\omega_{0}}{2 v_{t 0}} r_{0}^{2} \\
g_{2}=\frac{\omega_{0}}{2 v_{t 0}} r^{2}+r-f_{0} r_{0}-\frac{\omega_{0}}{2 v_{t 0}} r_{0}^{2} .
\end{array}\right.
$$


The criterion for a closed trajectory is that Equation 6.8 has a solution that satisfies $0<r_{\min } \leq r \leq r_{\max }<\infty$. If the solid body air domain were infinite, a particle would always have a closed orbit regardless of its size and corresponding terminal velocity. The reason is that the air velocity goes to infinity as $r \rightarrow \infty$. Therefore, no matter how large the terminal velocity is, there must be a region in which particles can be trapped. Figure 6.12 shows one trajectory for a specific case: $\omega=0.01$ $\mathrm{s}^{-1}$, and a particle with $v_{t 0}=0.1 \mathrm{~ms}^{-1}$ seeded at $x=50 \mathrm{~m}$ and $z=50 \mathrm{~m}$. From Equation 6.8 we can calculate the available range of $r$ : $68.1 \leq r \leq 88.1 m$ (grey region in Figure 6.12 a. Indeed, it is clear to see in Figure 6.12 b (black curve) that the trajectory is bounded by two circles with radii $r_{\min }=68.1 \mathrm{~m}$ and $r_{\max }=88.1 \mathrm{~m}$ - In addition, the radius of the bounded circle also represents the curvature at the intersection point between circle and trajectory curves. Consistent with previous observations, the results show that the radius of curvature is larger in the updraft region than that in the downdraft region. Figure 6.12 c shows three trajectories for particles with different size. Increasing the terminal velocity shifts the trajectory to a larger updraft velocity region, and to a smaller downdraft velocity region. All of these properties were previously observed in the calculated trajectories in previous section (in Figure 6.9). In reality, boundary-layer vortices are bounded and therefore the stable orbit radius has to be compared to the vortex size or, in the case of a Rankine vortex, the size of the vortex core. 

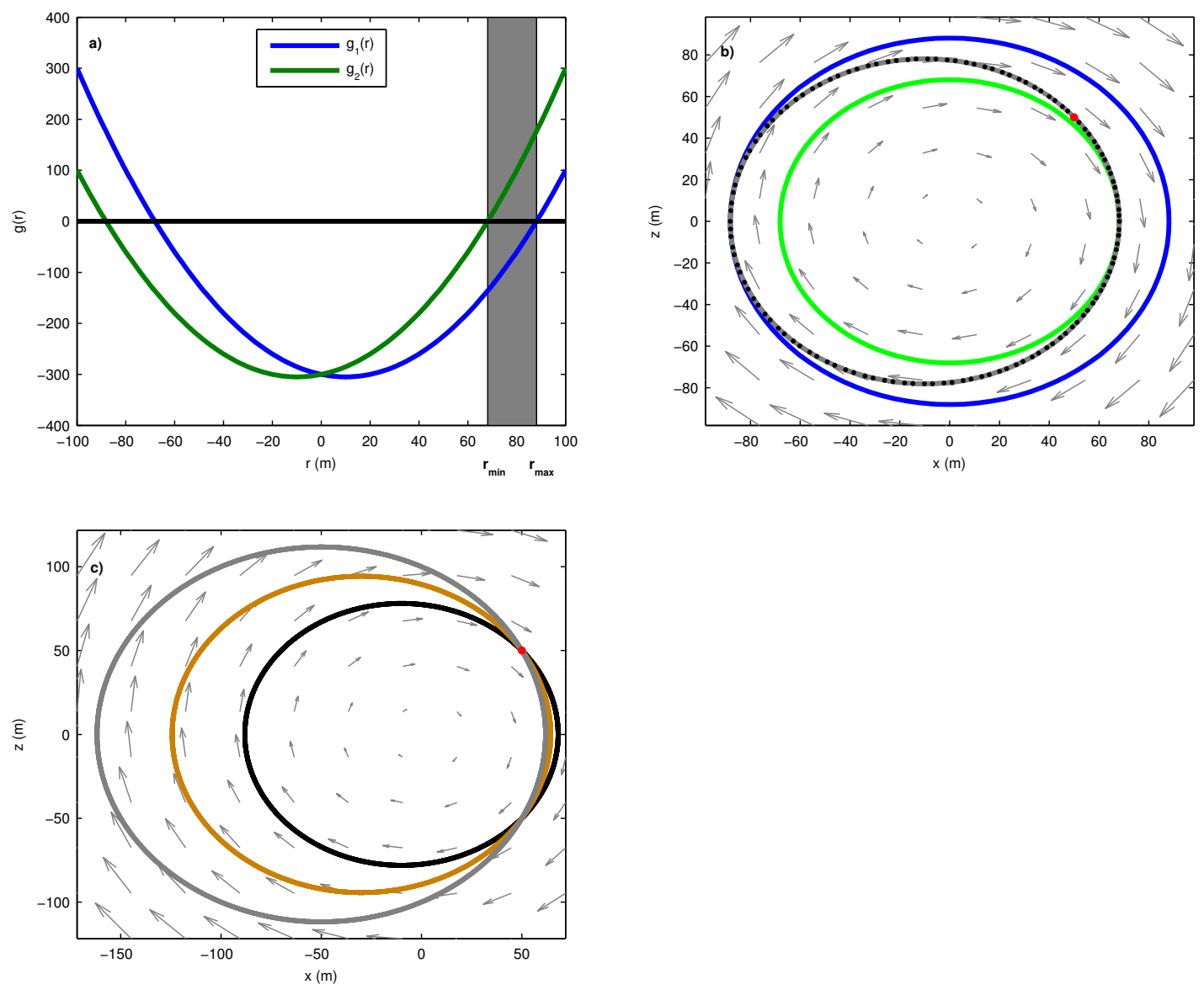

Figure 6.12: (a) Available range of $r$ for a closed trajectory in the solid body rotation field $v_{a, \theta}=\omega r$, where $\omega=0.01 s^{-1}$. Black line is $g(r)=0$. Details of $g_{1}, g_{2}, r_{\min }$ and $r_{\max }$ are described in the text. (b) Particle trajectory in the field (black dashed line, with blue and green circles denoting the limiting radii of curvature). Initial particle with constant terminal velocity $v_{t 0}=0.1 \mathrm{~ms}^{-1}$ is located at $x=50 \mathrm{~m}, z=50 \mathrm{~m}$. Green and blue lines are bounded circles with radii of $r_{\min }$ and $r_{\max }$. Gray dots are simulated trajectory, and black dots are from the analytical solution. Red point represents the initial location. (c) Trajectories of particles with three different terminal velocities $v_{t 0}=0.10 .3,0.5 \mathrm{~ms}^{-1}$. 


\section{Irrotational Field}

Outside the solid-body vortex core is an irrotational velocity field that decays with distance $r$. For the purposes of analysis, we consider a purely irrotational field defined as $v_{a, r}=0, v_{a, \theta}=\frac{B_{0}}{r}$, where $B_{0}$ is a constant. In this field, the velocity is infinity at the center of the vortex, and decreases to zero as $r \rightarrow \infty$. In practice, initial particle locations can be placed sufficiently far from the vortex core so as to approximate the outside of a Rankine vortex. The trajectory for a particle with the initial location $\left(r_{0}, \theta_{0}\right)$ can be solved analytically from Equation 6.5,

$$
f=-\frac{B_{0}}{v_{t 0} r} \ln r+\frac{f_{0} r_{0}+\frac{B_{0}}{v_{t 0}} \ln r_{0}}{r} .
$$

Following the same approach as in the solid body rotation field, from $-1 \leq f \leq 1$ we obtain two inequalities,

$$
\left\{\begin{array}{l}
g_{1} \leq \ln r \\
g_{2} \geq \ln r
\end{array}\right.
$$

where, 


$$
\left\{\begin{array}{l}
g_{1}=-\frac{v_{t 0}}{B_{0}} r+\ln r_{0}+\frac{f_{0} r_{0} v_{t 0}}{B_{0}} \\
g_{2}=\frac{v_{t 0}}{B_{0}} r+\ln r_{0}+\frac{f_{0} r_{0} v_{t 0}}{B_{0}}
\end{array}\right.
$$

It is not difficult to prove that $g_{1}$ will always intersect $\ln r$ in the region of $0<r<\infty$, but $g_{2}$ will not always intersect $\ln r$. From above we know that the criteria for a closed trajectory is the possible orbit radius should be bounded in a finite region. So the necessary and sufficient condition for a closed orbit is that the $g_{2}$ curve intersects with the $\ln r$ curve. We take $B_{0}=100 \mathrm{~m}^{2} \mathrm{~s}^{-1}, v_{t 0}=0.1 \mathrm{~ms}^{-1}$ as an example. The particle is initially located at $x=50 \mathrm{~m}$ and $z=50 \mathrm{~m}$ as before. The available range of $r$ is shown in Figure 6.13 a: $r_{\min } \leq r \leq r_{\max }$ where $r_{\min }$ is the intersection of $g_{1}$ and $\ln r$ and $r_{\max }$ is the intersection of $g_{2}$ and $\ln r$. As seen in Figure 6.13 b, black dashed line, the trajectory is bounded by two circles with radii of $r_{\min }=69.4$ $\mathrm{m}$ and $r_{\max }=80.6 \mathrm{~m}$. Trajectories for three particle sizes are shown in Figure $6.13 \mathrm{c}$. It is interesting to see that when $v_{t 0}=0.5 \mathrm{~ms}^{-1}$ the trajectory is not a closed orbit. Formally, the reason is that $g_{2}$ and $\ln r$ do not intersect in that case. Physically, it means the particle is so large (orbit radius increases with particle size) that the air can't manage to get the particle back into the updraft for a given $\left(r_{0}, \theta_{0}\right)$. Thus, if the vortex is in a region favorable to ice crystal growth, the particles will always eventually grow to a size where they can no longer be trapped due to their increasing fall speeds. Whether large fall speed, or size of the orbit compared to system boundaries (e.g., the ground), or the conditions favorable for growth versus 
evaporation eventually limit the ice crystal lifetime will depend on specifics of the thermodynamic and velocity fields. Two examples of changes in the velocity field relative to the thermodynamic fields are given in the following section in order to further explore implications.
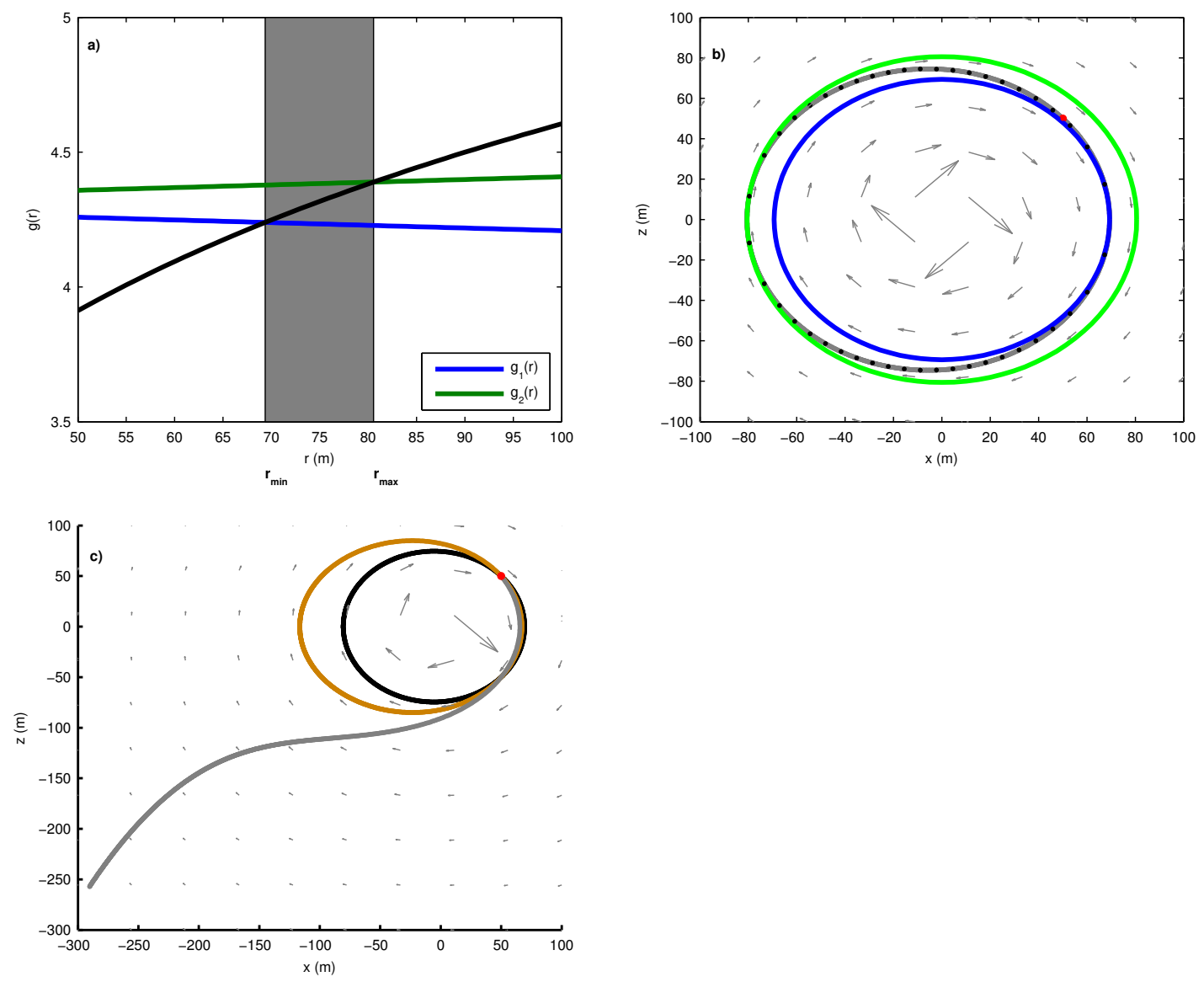

Figure 6.13: (a) Available range of $r$ for a closed trajectory in simple irrotational field $v_{a, \theta}=B_{0} / r$, where $B_{0}=100 \mathrm{~m}^{2} \mathrm{~s}^{-1}$. Black line is $g(r)=\ln r$. Details of $g_{1}, g_{2}, r_{\min }$ and $r_{\max }$ are described in the text. (b) Particle trajectory in the field (black dashed line, with blue and green circles denoting the limiting radii of curvature). Initial particle with constant terminal velocity $v_{t 0}=0.1 \mathrm{~ms}^{-1}$ is located at $x=50 \mathrm{~m}, z=50 \mathrm{~m}$. Symbols are the same as those in Figure 6.12. (c) Trajectories of particles with three different terminal velocities $v_{t 0}=0.10 .3,0.5 \mathrm{~ms}^{-1}$. 
In summary, gravitational settling will tend to drag a particle away from the center of vortex. In the solid body rotation field, the particle can always find a region where the air velocity is larger than its terminal velocity, because the air velocity goes to infinity as $r \rightarrow \infty$. In the irrotational field, there must be a region where particles cannot be recycled, because the air velocity goes to zero as $r \rightarrow \infty$. In general, to trap a particle in a convective eddy, the air velocity should be larger or at least close to the terminal velocity of particles. It should be mentioned that the constant terminal velocity assumption is definitely not valid for a real cloud. The reason we assume the terminal velocity is a constant is because we can then obtain an analytical solution for the particle's trajectory for a given velocity field. This allows us to investigate the stability boundaries and the role of the vortex structure in detail, albeit for an idealized system. Specifically, in Figure 6.8 and the previous section, we observed that Lagrangian ice particle tracks show quite sharp boundaries for the starting points of long lifetime particles. The analytical model introduced here provides an interpretation for that observed sharpness in stable trapping regions. 


\section{Ice Water Content In A Coupled Versus Decoupled}

\section{Boundary Layer}

How significant are the long lifetime particles for the ice water content of a mixedphase cloud? Clearly the answer to this question depends on both the velocity flow field and the thermodynamic profile within the turbulent layer. To investigate this we proceed by holding the thermodynamic profile fixed and considering the effect of changing the mixed layer depth. From the results obtained thus far, we know that small ice particles can be trapped in the eddy structure, and that whether the trapped ice particles can grow or sublimate depends on the ice supersaturation profile within the trapping region. Specifically, we assumed that the eddy extends from the top of the boundary layer all the way to the ground (see Figure 6.6). This structure is representative of a coupled boundary layer. It should be mentioned that in reality, a coupled boundary layer is usually driven, to a large extent, by surface sensible and latent heat fluxes, which are ignored in our simple kinematic model. In our case, the eddy was assumed to be fully developed between 0 and $800 \mathrm{~m}$. The processes that lead to coupling or decoupling are complex, and for the purposes of this paper we are guided by the LES and simply take the structure as a given. LES and observational studies of other cases often show the existence of shallow mixed-phase clouds within

elevated mixed layers decoupled from the surface [96, 119], in which case air motions 
are usually driven by cloud-top radiative cooling. An analysis of the dynamics (or energetics) of such mixed layers is also beyond the scope of this paper. Here to investigate how the decoupled flow field affects the recycling ice particles, we simply limit the depth of the eddy to the layer between $400 \mathrm{~m}$ and $800 \mathrm{~m}$ as shown in Figure 6.14. Ice particles are seeded within the mixed-phase region, between 600 $\mathrm{m}$ and $800 \mathrm{~m}$ in the decoupled field, with the same spatial distribution as before. Thermodynamic profiles used in the decoupled field are the same as in the coupled field. This implies that for the decoupled state almost the entire turbulent layer lies within the ice saturated region.

Several cases are investigated for both coupled and decoupled field. For the base case 'AR2, coupled, $2.0 \mathrm{~ms}^{-1}$ ', the aspect ratio of the eddy (width/height) is about 2 , and the large eddy propagates down to the surface with $v_{\max }$ of $2.0 \mathrm{~ms}^{-1}$. In this case, there are two large eddies in the domain, as shown in Figure 6.6. To investigate the effect of spatial structure of the turbulent fields, two more coupled fields with narrower eddies are considered: 'AR1, coupled, $2.0 \mathrm{~ms}^{-1}$ ' and 'AR0.5, coupled, 2.0 $m s^{-1}$, corresponding to 4 eddies and 8 eddies in the domain respectively. For the decoupled cases, we consider 'AR4, decoupled, $2.0 \mathrm{~ms}^{-1}$ ', 'AR2, decoupled, $2.0 \mathrm{~ms}^{-1}$, and 'AR1, decoupled, $2.0 \mathrm{~ms}^{-1}$ ', which represents 2,4 and 8 eddies in the decoupled field. As the air velocity in the decoupled field might be smaller than that in the coupled field, three more decoupled cases with the $v_{\max }$ of $0.5 \mathrm{~ms}^{-1}$ are also investigated. 


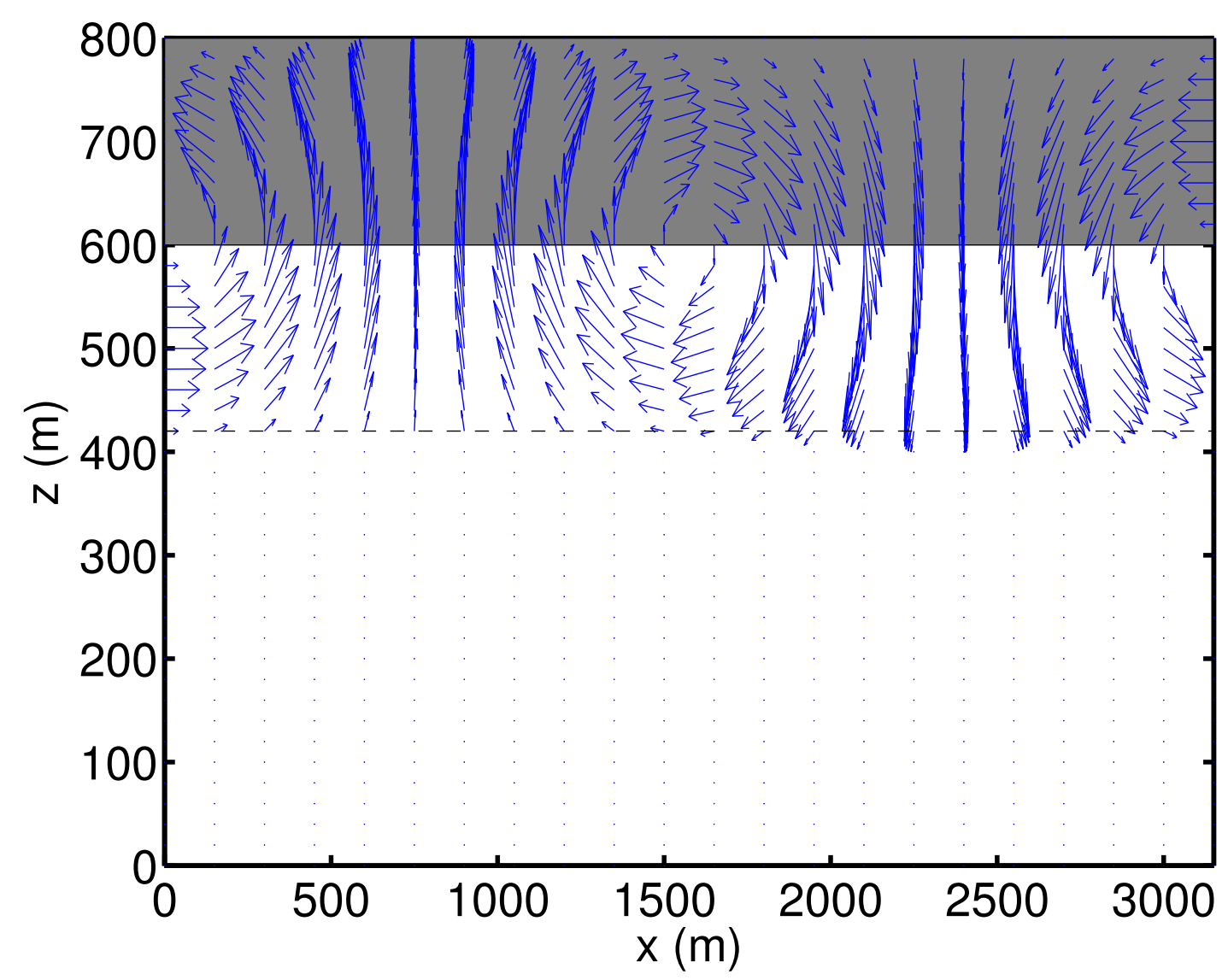

Figure 6.14: Velocity field when the eddy is decoupled from the surface. The eddy is developed between $400 \mathrm{~m}$ and $800 \mathrm{~m}$, and below $400 \mathrm{~m}$ the air is still. The black dashed line represents where the environment is saturated with respect to ice, and the shaded area denotes the mixed-phase region.

The total ice water mass in the mixed phase cloud for different cases is compared with that in the base case. It should be mentioned that since the mixed-phase cloud geometry is the same in all cases, the total ice water mass is equivalent to the cloud-mean ice water content for the purpose of the presented analysis. Here ice particles are seeded continuously between $600 \mathrm{~m}$ and $800 \mathrm{~m}$ every two seconds in all fields. Results are shown in Figure 6.15 a. It can be seen that the total ice water 

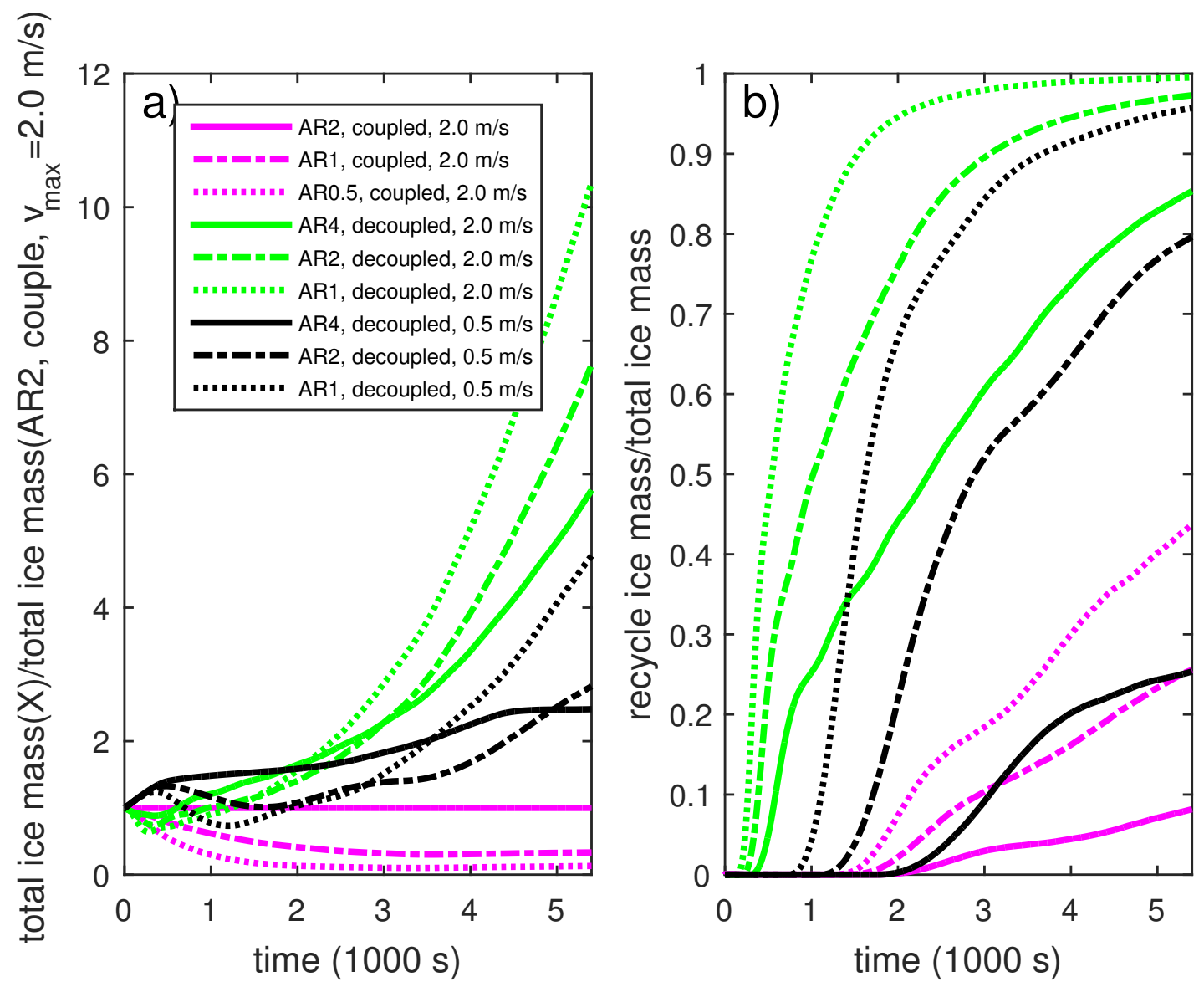

Figure 6.15: a) Time variation of total ice water mass ratio in the mixed phase cloud (between $600 \mathrm{~m}$ and $800 \mathrm{~m}$ ) for different cases with respect to the base case: AR2, coupled, $v_{\max }=2.0 \mathrm{~m} / \mathrm{s}$. Case details are described in the text. b) The mass fraction of recycling ice particles in the mixed phase cloud for different cases.

mass ratio (with respect to 'AR2, coupled, $2.0 \mathrm{~ms}^{-1}$ ') is still increasing with time for 'AR4, decoupled, $2.0 \mathrm{~ms}^{-1}$ ' case, but it has stabilized to nearly a constant for 'AR4, decoupled, $0.5 \mathrm{~ms}^{-1}$ ' case. This can be explained by the significant change of the $\chi$-lifetime relationship shown in Figure 6.16, where $\chi$ is larger than 0.25 after 1.5 hours for 'AR4, decoupled, $2.0 \mathrm{~ms}^{-1}$ ' case. For the 'AR4, decoupled, $0.5 \mathrm{~ms}^{-1}$ ' case, $\chi$ is even larger than for the 'AR2, coupled, $2.0 \mathrm{~ms}^{-1}$ ' case at the beginning, because 
ice particles can stay in the cloud longer due to the small air velocity. But $\chi$ decays rapidly after $1000 \mathrm{~s}$, because the small updraft velocity cannot trap large ice particles.

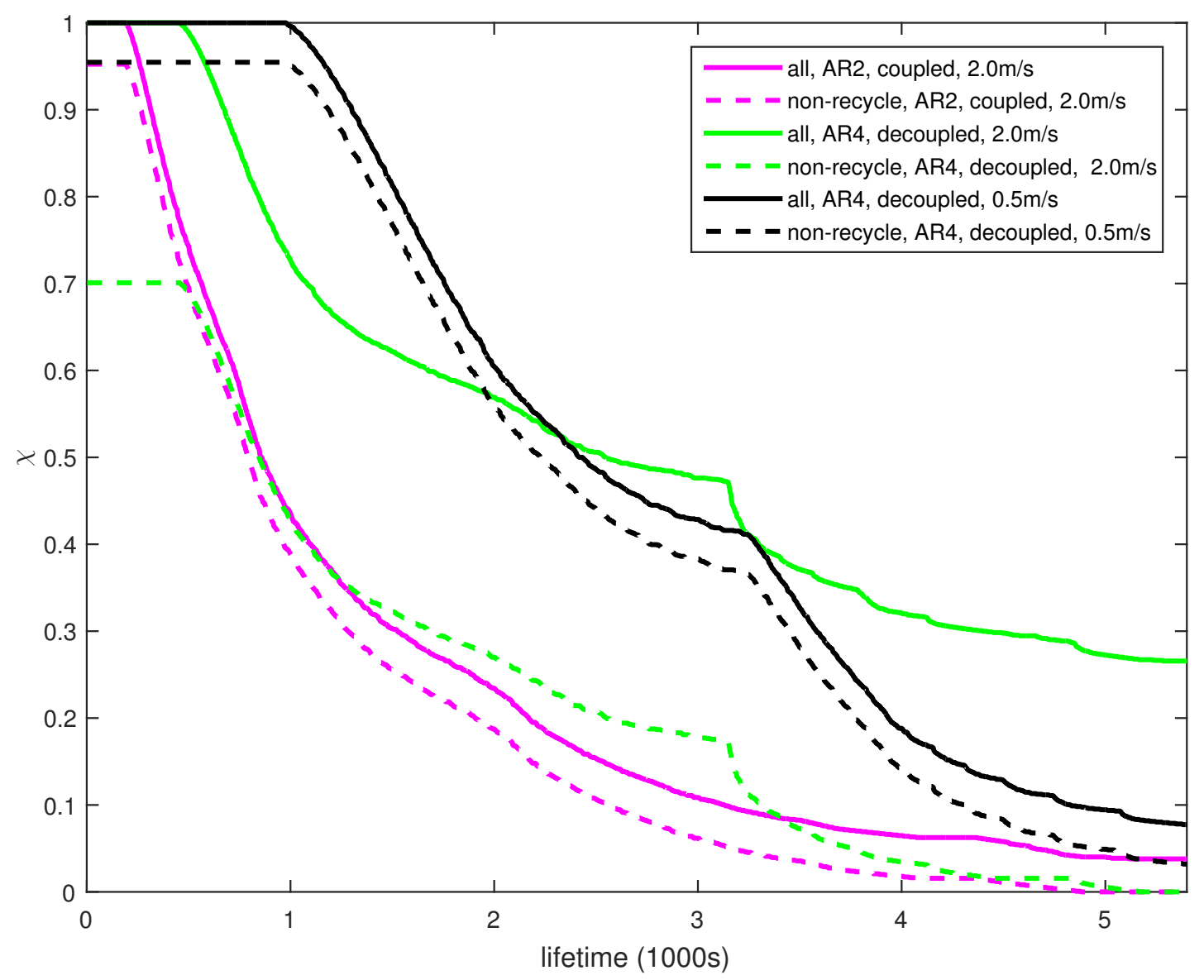

Figure 6.16: $\chi$ versus lifetime for all ice particles (solid line) and nonrecycle ice particles (dashed line). Different colors represent different cases: magenta is for the 'AR2, coupled, $2.0 \mathrm{~ms}^{-1}$ ' case, green line is for 'AR4, decoupled, $2.0 \mathrm{~ms}^{-1}$ ' case, and black is for 'AR4, decoupled, $0.5 \mathrm{~ms}^{-1}$ ' case.

In general, the ratio is larger for decoupled case with $v_{\max }$ of $2.0 \mathrm{~ms}^{-1}$ than that for decoupled case with $0.5 \mathrm{~ms}^{-1}$, while the latter is larger than that for coupled case with $2.0 \mathrm{~ms}^{-1}$. For example, the total ice water mass in the mixed phase cloud for 
'AR4, decoupled, $2.0 \mathrm{~ms}^{-1}$ ' is almost six times larger than that for the base case after 1.5 hours. Even when the velocity strength in the decoupled field $\left(0.5 \mathrm{~ms}^{-1}\right)$ is much smaller than that in the coupled field, the ratio can still be larger than 2 . This is because in the coupled field the trapping region is between 0 and $800 \mathrm{~m}$, but ice particles can only grow when the environment is supersaturated with respect to ice, which is above $420 \mathrm{~m}$ in our case (above the black dashed line in Figure 6.6). Therefore, only ice particles that are sufficiently large or that have just the right initial conditions can be recycled before they totally sublimate. For the decoupled field (shown in Figure 6.14) ice particles can only be trapped between $400 \mathrm{~m}$ and $800 \mathrm{~m}$. The environmental air is supersaturated with respect to ice almost over the whole trapping region and therefore the recycled ice particles grow continuously until they become large enough to fall out of the mixed layer. The recycled particles, thus have longer lifetime.

However, trends of the total ice water mass ratio when narrowing the eddies' widths are different for coupled and decoupled cases, as shown in Figure 6.15 a. The ratio increases as we decrease the eddies' width for the decoupled cases, especially for $2.0 \mathrm{~ms}^{-1}$, but decreases for the coupled cases. This might be related to the relative importance of particles' horizontal transport time and sublimation time. The horizontal transport time describes the time needed for ice particles to be transported from updraft/downdraft region to downdraft/updraft region, which depends on the kinematic properties of the field. Sublimation time describes the time 
needed for ice particles to be sublimated, which depends on both the thermaldynamic properties of the field and particles' properties. When narrowing the eddies' width, the horizontal transport time decreases due to the smaller distance between updraft and downdraft, which is favourable for particle trapping. This can be verified from Figure $6.15 \mathrm{~b}$, where the fraction of recycling ice particles increases when narrowing the eddies' width for all conditions. Therefore, more ice particles are recycled when decreasing the eddies' width. For the decoupled cases, the tracking region is almost supersaturated with respect to ice. Ice particles can growth nearly all the time, until they're large enough to be recycled. Therefore, the ratio increases as we decreases the eddies' width. Things are complicated for the coupled cases, because part of the tracking region is subsaturated with respect to ice. Although narrowing the eddies width decreases the transport time, it also decreases the radii of ice particles falling into the ice subsaturated region, thus decreases the evaporation time.

Finally, to understand how important the recycling ice particles are, we need to determine the fraction of ice water mass for recycling particles in the mixed phase cloud. Assuming ice particles are seeded continuously between $600 \mathrm{~m}$ and $800 \mathrm{~m}$, mass fractions of recycling particles for coupled and decoupled fields are shown in Figure 6.15 b. It can be seen that the recycling ice particle mass fraction can be up to 0.9 after 1.5 hours for 'AR4, decoupled, $2.0 \mathrm{~ms}^{-1}$ ' case, and around 0.25 for 'AR4, decoupled, $0.5 \mathrm{~ms}^{-1}$ ' case, but it is only 0.1 for 'AR2, coupled, $2.0 \mathrm{~ms}^{-1}$ ' case. 
Therefore, the recycling ice particles contribute more to the ice water content in the decoupled field. Of course, the fraction also depends on the seeding frequency. If ice particles are only seeded at the beginning, the fraction can still reach 1.0 after one hour even for coupled field, as shown in Figure 6.7 e.

\section{Conclusions}

The purpose of this study is to understand dynamical factors that govern the ice microphysics of thin, mixed-phase stratiform clouds, beyond the known importance of ice nucleation and vapor deposition rates. We were motivated by prior work focused on the microphysical consequences of stochastic ice nucleation distributed throughout

a mixed-phase cloud [148, 149]. During the course of that work it became clear that both LES and observations often reveal the presence of ice crystals significantly larger than can be easily explained with idealized models. In this study we have taken a Lagrangian approach, considering the time dependent thermodynamic and turbulent structure of the boundary layer, while tracking ice crystals as they nucleate, grow by vapor deposition, and eventually either fall to the ground as precipitation or sublimate. The focus has been to understand the ice crystal trajectories that lead to long ice crystal residence times within the mixed-phase regions, which in turn usually correspond to the largest ice crystals in the cloud. 
We seed ice particles in a mixed phase cloud with a 3-D dynamic velocity field generated by a LES cloud model. Ice particle trajectories are calculated using a Lagrangian tracking method, and their growth depends on the environmental saturation ratio with respect to ice. Surprisingly, more than $10 \%$ of the ice particles still survive after 1.5 hours, much longer than the large eddy turnover time or the time for an ice particle started at the top of a cloud to fall out in still air. We find that many of the long lifetime ice particles are recycled several times in and out of the mixed-phase region before they totally sublimate or fall to the ground.

To investigate the origin locations of the long lifetime ice particles, we seed ice particles in an idealized $2 \mathrm{D}$ velocity field. We observe two types of long lifetime ice particle trajectories: quasi-steady growth, in which an ice particle is suspended in an updraft region near a stagnation point for a long time, and then falls out; and recycled, in which an ice particle falls from the cloud and is swept back into the cloud several times, experiencing steady growth. The number of recycling ice particles and the recycling times are sensitive to the flow velocity amplitude. It is shown that both the fraction and recycling times increase in faster flow.

Trajectories in both 2-D and 3-D fields show that ice particles can have long lifetime when they are suspended in the updraft region (stagnation point), or recycled 
several times. Ice particles can be recycled when they are trapped in the large eddy structure, and whether they grow or sublimate depends on the environmental saturation ratio in the trapping region. Many ice particles will totally sublimate in the sub-cloud region, but a fraction of the ice particles can be recycled back into the cloud before they totally sublimate. Those lucky ice particles experience steady growth for each recycling cycle, until they fall to the ground when they are too large to be trapped in the eddy.

To understand the conditions required for ice particle trapping, analytical solutions for closed particle orbits are obtained for three idealized velocity fields, assuming constant particle size. Results show that particles can always be trapped in the solid-body rotation fluid field, but not always in irrotational fluid field. This is consistent with the expectation that an ice particle cannot be recycled when its terminal velocity is larger than the air updraft velocity. Thus, if thermodynamic conditions support net growth over a closed orbit, trapped ice particles will tend to grow until their terminal fall speed reaches the maximum vertical velocity in the cloud. Alternatively, the orbit will grow until it is no longer confined within the large eddy circulation, or until it grows to occupy drier regions of the mixed layer so that no net growth occurs. 
Are the results of the Lagrangian model aligned with observations? For example, we observe that stronger updraft velocity leads to more recycling ice particles with longer lifetime and larger sizes. This is somewhat challenging because we are not aware of any studies of correlation between crystal size and updraft strength on a scale of a single cloud eddy. Our understanding is that current in situ and remote sensing measurements do not have the time (sampling) resolution to look at such detail. However, [116] developed a conceptual diagram based on their observations, showing ice in the updraft and virtually no ice in the downdraft, although the horizontal scale is, at least, an order of magnitude larger than our eddies. [115] have also shown some observational evidence that the stronger vertical motions correlate with larger ice water path. The differing scales between these results and our simulations does not allow for quantitative comparison, but can be considered as suggestive evidence.

It is commonly observed that mixed phase stratiform clouds exist in turbulent layers that are decoupled from the surface, for example when cloud-top radiative cooling is the primary driver of mixing. Within the 2-D kinematic model we approximated a decoupled velocity field as an eddy confined to the layer between $400 \mathrm{~m}$ and $800 \mathrm{~m}$, compared to the coupled field between $0 \mathrm{~m}$ and $800 \mathrm{~m}$. The thermodynamic profile was taken to be the same in both cases. Results show that both the spatial structure of the eddies and the velocity strength affect the microphysical properties of the 
mixed phase cloud. In general, the total ice water mass in the mixed phase cloud for the decoupled field is larger than that for the coupled field. In addition, the recycling ice particles are more important in the decoupled field: the ice water mass fraction for the recycling ice particles in the decoupled field can reach 0.9 after 1.5 hours, while, it's only 0.1 in the coupled field. The reason is that most of the trapping region in the decoupled field is supersaturated with respect to ice, which sustains the growth of ice particles. In contrast, only half of the trapping region in the coupled field is supersaturated, thus only a relatively small number of ice particles can be recycled, while others will totally sublimate in the subsaturated region. The horizontal transport time scale, which depends on the kinematic properties of the field, and the sublimation time scale, which depends on the thermaldynamic properties of the field, are discussed qualitatively to explain the different responses of total ice water mass to narrowing the eddies' size for the coupled and decoupled cases.

Previous studies show that the ice fraction of total condensed water mass in mixed phase clouds depends on several variables, including temperature, relative humidity, cloud type and aerosol type [52, 53, 89]. The nucleation efficiency and the Bergeron process have been used as explanations for the wide range of observed ice water content in mixed phase clouds. Our results show that even if the cloud temperature, relative humidity, liquid water content, cloud thickness and volume nucleation rate are all the same, ice water mass in the mixed phase cloud for a decoupled field 
can be larger than that for a coupled field, even if the turbulence in the decoupled field is much weaker than in the coupled field. This provides another possible mechanism to explain the change of ice water content: the variation of the dynamic and thermodynamic properties of the mixed layer, which directly influences the recycling of ice particles.

\section{Acknowledgments}

This research was supported by the DOE Office of Science as part of the Atmospheric System Research program, including through grant DE-SC0006949. Large eddy simulations were performed using PNNL Institutional Computing at Pacific Northwest National Laboratory. The Pacific Northwest National Laboratory is operated for DOE by Battelle Memorial Institute under contract DE-AC05-76RL01830. 


\section{Chapter 7}

\section{Discussion}

\section{Conclusion}

The research described in this thesis is mainly focused on the two common ice nucleation modes in the atmosphere: contact mode and immersion mode. The two key questions being investigated are:

* Why is contact nucleation more efficient than immersion nucleation?

* Is time important for ice formation in the atmosphere?

Chapters 2 and 3 are related to the first question. Our experiments show that ice 
nucleation can be triggered around the edge of a supercooled drop through electrowetting or vibration. High speed video confirms that ice nucleation always starts near the contact line, and can occur at multiple points. The freezing is strongly related to the existence of a locally curved contact line due to either electrowetting or inhomogeneous pinning of the substrate. We propose that the locally curved contact line can produce local pressure perturbations at the contact line. Given the waterice density anomaly, a negative pressure perturbation can increase the driving force for phase change, thus enhance the probability of ice nucleation. Our theoretical calculation shows that a suppression of ice nucleation rate resulting from a $\sim 10$ $\mathrm{K}$ temperature increase can be balanced by a negative pressure of $\sim 10^{8} \mathrm{~Pa}$. This negative pressure could be achievable through contact distortion due to a collision or other disruption, or due to surface roughness or cavity collapse. Pressure induced ice nucleation provides another way to interpret why contact nucleation is more efficient than immersion nucleation. The quantitative expression provided in Chapter 3 might be useful to predict ice formation in the atmospheric models, but further research will be required to better understand the causes and typical magnitudes of pressure perturbations.

Chapters 4, 5 and 6 are related to the second question. Modeling results show that time is important in mixed-phase stratiform clouds for two reasons. First, time is important for ice nucleation. To balance the loss of ice particles due to precipitation, 
there must be a source of ice nuclei. One possibility is that ice particles are generated slowly and stochastically from the much larger population of supercooled water droplets. Although this hypothesis can explain the existence of long lifetime mixedphase stratiform clouds, there is no direct evidence to prove that time-dependent ice nucleation is important in real clouds due to the short sample residence time of current instruments. We address the problem from another point of view: if ice is generated steadily and stochastically, what microphysical properties would the mixedphase stratiform clouds be expected to have? Our minimalist model shows that there would be a 2.5 power-law relationship between ice water content and ice number concentration, as long as the volume ice nucleation rate is constant. Because ice water content and ice number concentration in mixed phase stratiform clouds can be obtained from in-situ measurements, their relationship can provide a hint of where the ice comes from, and the derived volume ice nucleation rate can be linked to ice nucleation rates obtained in the field or in the lab.

Second, time is also important for ice growth. Ice particles can survive much longer time in a turbulent environment than in still air. Our Lagrangian ice particle tracking results confirm that long lifetime ice particles exist in mixed-phase stratiform clouds. Small ice particles can be trapped in the eddy-like structures, and then whether those ice particles grow or sublimate depends on the thermodynamic field in the trapping region. The coupling effect between dynamic and thermodynamic properties of the boundary layer decides how long ice particles can survive and how large they can 
grow. Our results can explain the fast phase-partition change observed in the mixed phase cloud [53].

\section{Future Research}

We started the electrowetting experiments with the aim of studying the electric field effect on ice nucleation. Although our results show that an electric field of magnitude less than $5 \mathrm{~V} / \mu \mathrm{m}$ alone has no significant effect on ice nucleation, recent experimental results give a hint that strong electric fields might enhance ice nucleation rates [12, 27]. More experiments can be done to further explore this effect, but new techniques will be required.

Whether electric current will trigger ice nucleation is also an interesting question. Previous studies have shown that electric current can trigger ice nucleation

[95, 112, 113]. However, our own preliminary experiments with droplets on silicon substrates gave negative results: no freezing occurs even at $-15{ }^{\circ} \mathrm{C}$ with electric current. More experiments are needed to investigate the role of electric current on ice nucleation in the future. 
Another interesting topic related to electric current is whether bubble formation and/or collapse can trigger ice nucleation. Our electric-current experiments show that although many bubbles are produced through electrolysis of water, no ice nucleation occurs. However, previous studies show that ultrasound can trigger ice nucleation, and the mechanism is believed to be related to the formation of cavitation bubbles [17, 154]. So it would be interesting to figure out why bubbles cannot trigger ice nucleation in our experiment or to clarify the mechanism of nucleation that occurs in the ultrasound experiments..

Ice nucleation is usually considered under conditions of constant temperature and pressure. However, nucleation might occur at nonisothermal conditions due the release of latent heat [143]. Evidence has been found in the gas phase, e.g. homogeneous nucleation of water vapor [6]. It is therefore also worthwhile to investigate whether nonisothermal conditions influence nucleation in the liquid phase. Early in this work preliminary experiments on silicon and silicon-dioxide substrates. It was expected that the freezing temperatures on these two substrates would be different, because the thicknesses of the oxidation layers are different and therefore the rate of heat dissipation would be different. However, the results were negative: there was no detectable difference in freezing temperature. More theoretical and laboratory work can be done to investigate this possible effect in the future. 
With the high speed camera, we can not only detect the ice nucleation site inside a water droplet, but also we can obtain the speed of crystallization. This might be related to sea ice propagation and food preservation [67, 102]. My preliminary experiments show that ice propagation speed can be affected by temperature, substrate and electric field, which is consistent with previous studies [43, 98]. More research can be done in the future to quantify crystallization speed under different conditions.

Another interesting phenomenon was observed when freezing occurs on a silicon substrate above $-18^{\circ} \mathrm{C}$. We find that several new ice nucleation sites can appear in front of the main water-ice interface during the crystallization process, as shown in Figure 7.1, In addition, those new ice particles usually have a rectangular shape, and the long side is roughly perpendicular to the water-ice interface. There are two interesting questions:(1) Are these newly appearing ice nucleation sites due to the propagation of a pressure wave, or due to the propagation of a thin layer of ice on the substrate? (2) Why is the long side always perpendicular to the water-ice interface? More experiments are needed to answer these questions in the future.

Our electrofreezing and vibration experiments suggest that negative pressure perturbations in a water droplet would enhance the ice nucleation rate. More experiments 


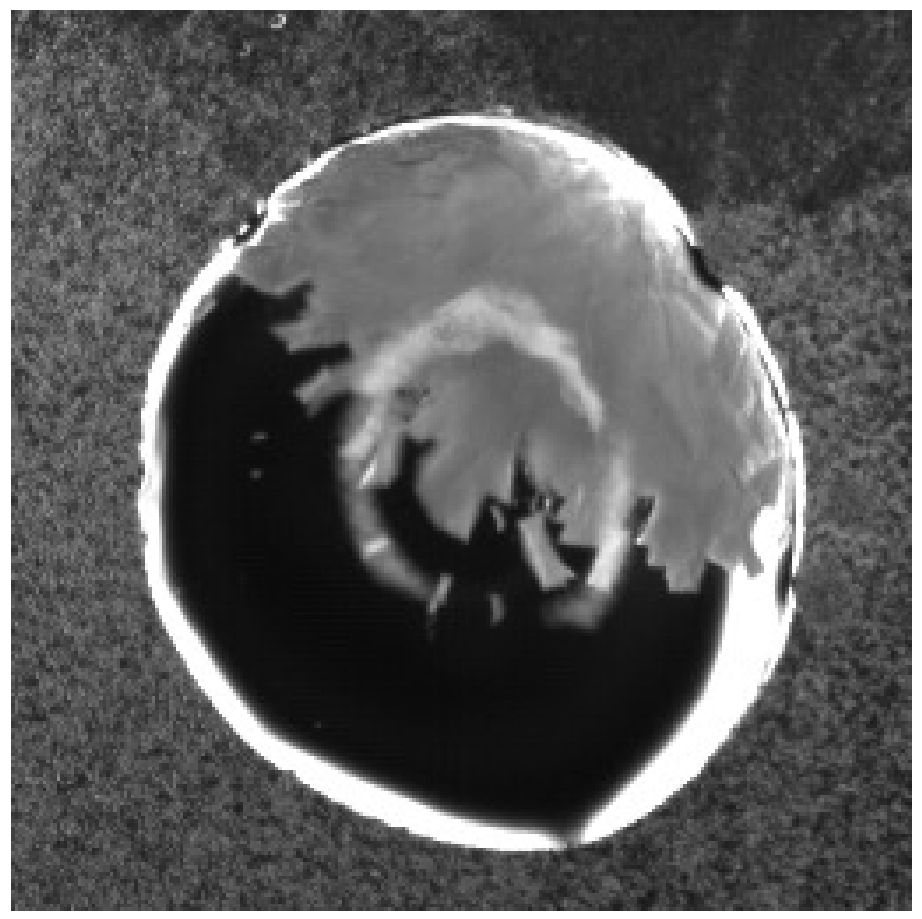

Figure 7.1: Ice formation in front of the main water-ice interface. The water droplet is freezing at $-14{ }^{\circ} \mathrm{C}$ on a silicon substrate.

should be done to quantify the pressure perturbations occurring during collisions between droplets and a substrate or particle, their quantitative enhancement of the nucleation rate, and their implications for the phenomenon of contact nucleation. The proposed experiments include impaction of supercooled droplets on a supercooled substrate with controlled speed, collision between two supercooled droplets with controlled speed, or simulating the pressure perturbation that occur in coalescing water droplets.

Another interesting topic is to study the ice multiplication process in water droplets on a cold stage. Recent laboratory experiments show that the formation of ice 
shell can lead to droplet explosion [139]. In contrast, we have not observed droplet explosion during our freezing experiments. Superhydrophobic substrates might be needed to investigate this phenomenon.

In Chapters 4 and 5, we predict that ice water content and ice number concentration in mixed-phase stratiform clouds have a 2.5 power-law relationship, as long as the volume ice nucleation rate is constant. The 2.5 exponent depends on the growth behavior of ice particles. In our study, we assume the shape factor is 1 , which is spherical. In reality, ice particles might be plane-like or column-like shapes, which would have different shape factors, thus change the power-law relationship. It would be useful to investigate the effect of ice growth habit on the power-law relationship in the future.

\section{Additional Research Topics}

Although not included as part of this thesis, additional research during my graduate work was done on two topics. Eventually, these may be extended to mixed-phase conditions, so they are briefly mentioned here. The first study involved entrainment and mixing in warm cloud. This work is published in full form in the journal 
Atmospheric Chemistry and Physics [151], and the abstract is included here. Cloud droplet response to entrainment and mixing between a cloud and its environment is considered, accounting for subsequent droplet growth during adiabatic ascent following a mixing event. The vertical profile for liquid water mixing ratio after a mixing event is derived analytically, allowing the reduction to be predicted from the mixing fraction and from the temperature and humidity for both the cloud and environment. It is derived for the limit of homogeneous mixing. The expression leads to a critical height above the mixing level: at the critical height the cloud droplet radius is the same for both mixed and unmixed parcels, and the critical height is independent of the updraft velocity and mixing fraction. Cloud droplets in a mixed parcel are larger than in an unmixed parcel above the critical height, which we refer to as the "super-adiabatic" growth region. Analytical results are confirmed with a bin microphysics cloud model. Using the model, we explore the effects of updraft velocity, aerosol source in the environmental air, and polydisperse cloud droplets. Results show that the mixed parcel is more likely to reach the super-adiabatic growth region when the environmental air is humid and clean. It is also confirmed that the analytical predictions are matched by the volume-mean cloud droplet radius for polydisperse size distributions. The findings have implications for the origin of large cloud droplets that may contribute to onset of collisioncoalescence in warm clouds.

The second study involved simulation of the turbulence and microphysical properties 
in a laboratory cloud chamber. The cloud chamber is a multiphase, turbulent reaction chamber at Michigan Technological University [15]. It has been used to study aerosol-cloud interactions in a turbulent environment [14]. My research involvement was to simulate the cloud chamber using LES. The original model is the LES code System for Atmospheric Modeling or SAM, which has been widely used to study warm and cold cloud processes in the atmosphere [55]. We modified SAM to mimic the Rayleigh-Benard convection in the cloud chamber [14]. The simulation domain is a rectangular box $\left(L_{x}=2 \mathrm{~m}, L_{y}=2 \mathrm{~m}\right.$ and $\left.L_{z}=1 \mathrm{~m}\right)$ with a resolution of $d x=d y=d z=0.03125 \mathrm{~m}$. The bottom surface is warmer than the top surface to drive the convection. Both bottom and top surfaces are assumed to be $100 \%$ saturated with respect to water and the surface fluxes (heat, water vapor, and momentum) are parameterized based on Monin-Obukhov similarity theory. In addition, we change the original periodic boundary condition of the atmospheric code to a fixed wall boundary condition appropriate for a closed chamber. The four side walls provide momentum flux (no-slip boundary) but no heat and water vapor fluxes. The initial temperature and water-vapor mixing ratio are set to linearly decrease with height. The cloud microphysical processes are simulated with a bin microphysics code with 33 mass-doubling bins for aerosol and 33 mass-doubling bins for cloud droplets [54]. The activation of aerosol particles, condensational growth of cloud droplets, collision-coalescence between cloud droplets, and sedimentation of cloud droplets are considered in the model. The results of the study are not 
detailed here, but briefly, the LES played a key role in verifying 1) that collisioncoalescence was not significant in causing the observed broadening of cloud droplet size distributions; and 2) that the LES was able to support, along with the measurements, the hypothesized mechanism of turbulence-induced broadening through supersaturation fluctuations. It is likely that this will continue to be an important tool for interpreting cloud chamber results and linking them to real atmospheric flows. 



\section{References}

[1] G. Adler, T. Koop, C. Haspel, I. Taraniuk, T. Moise, I. Koren, R. H. Heiblum, and Y. Rudich. Formation of highly porous aerosol particles by atmospheric freeze-drying in ice clouds. Proceedings of the National Academy of Sciences, 110(51):20414-20419, 2013.

[2] J. D. Atkinson, B. J. Murray, M. T. Woodhouse, T. F. Whale, K. J. Baustian, K. S. Carslaw, S. Dobbie, D. OSullivan, and T. L. Malkin. The importance of feldspar for ice nucleation by mineral dust in mixed-phase clouds. Nature, 498(7454):355-358, 2013.

[3] A. Avramov, A. S. Ackerman, A. M. Fridlind, B. van Diedenhoven, G. Botta, K. Aydin, J. Verlinde, A. V. Korolev, J. W. Strapp, G. M. McFarquhar, et al. Toward ice formation closure in arctic mixed-phase boundary layer clouds during isdac. Journal of Geophysical Research: Atmospheres, 116(D1), 2011. 
[4] A. Avramov and J. Y. Harrington. Influence of parameterized ice habit on simulated mixed phase arctic clouds. Journal of Geophysical Research: Atmospheres, 115(D3), 2010.

[5] M. Baker. Cloud microphysics and climate. Science, 276(5315):1072-1078, 1997.

[6] D. Barschdorff. Carrier gas effects on homogeneous nucleation of water vapor in a shock tube. The Physics of Fluids, 18(5):529-535, 1975.

[7] I. Borzsák and P. T. Cummings. Electrofreezing of water in molecular dynamics simulation accelerated by oscillatory shear. Physical Review E, 56(6):R6279, 1997.

[8] I. Borzsák and P. T. Cummings. Effect of oscillatory shear on the fluid-solid phase transition of supercooled water. Fluid phase equilibria, 150:141-149, 1998.

[9] I. Braslavsky and S. Lipson. Electrofreezing effect and nucleation of ice crystals in free growth experiments. Applied physics letters, 72(2):264-266, 1998.

[10] K. Browning and G. Foote. Airflow and hail growth in supercell storms and some implications for hail suppression. Quarterly Journal of the Royal Meteorological Society, 102(433):499-533, 1976.

[11] W. Cantrell and A. Heymsfield. Production of ice in tropospheric clouds: A review. Bulletin of the American Meteorological Society, 86(6):795, 2005. 
[12] K. Carpenter and V. Bahadur. Electrofreezing of water droplets under electrowetting fields. Langmuir, 31(7):2243-2248, 2015.

[13] G. Carrió, H. Jiang, and W. Cotton. Impact of aerosol intrusions on arctic boundary layer clouds. part i: 4 may 1998 case. Journal of the atmospheric sciences, 62(9):3082-3093, 2005.

[14] K. K. Chandrakar, W. Cantrell, K. Chang, D. Ciochetto, D. Niedermeier, M. Ovchinnikov, R. A. Shaw, and F. Yang. Aerosol indirect effect from turbulence-induced broadening of cloud-droplet size distributions. Proceedings of the National Academy of Sciences, 113(50):14243-14248, 2016.

[15] K. Chang, J. Bench, M. Brege, W. Cantrell, K. Chandrakar, D. Ciochetto, C. Mazzoleni, L. Mazzoleni, D. Niedermeier, and R. Shaw. A laboratory facility to study gas-aerosol-cloud interactions in a turbulent environment: The $\pi$ chamber. Bulletin of the American Meteorological Society, (2016), 2016.

[16] K. Chang, B. Malec, and R. Shaw. Turbulent pair dispersion in the presence of gravity. New Journal of Physics, 17(3), 2015.

[17] R. Chow, R. Blindt, R. Chivers, and M. Povey. A study on the primary and secondary nucleation of ice by power ultrasound. Ultrasonics, 43(4):227-230, 2005.

[18] W. A. Cooper. A possible mechanism for contact nucleation. Journal of the Atmospheric Sciences, 31(7):1832-1837, 1974. 
[19] W. Cui, M. Zhang, X. Duan, W. Pang, D. Zhang, and H. Zhang. Dynamics of electrowetting droplet motion in digital microfluidics systems: From dynamic saturation to device physics. Micromachines, 6(6):778-789, 2015.

[20] J. A. Curry, J. L. Schramm, W. B. Rossow, and D. Randall. Overview of arctic cloud and radiation characteristics. Journal of Climate, 9(8):1731-1764, 1996.

[21] G. Dawson and G. Cardell. Electrofreezing of supercooled waterdrops. Journal of Geophysical Research, 78(36):8864-8866, 1973.

[22] P. de Gennes, D. Quéré, F. Brochard-Wyart, and A. Reisinger. Capillarity and Wetting Phenomena: Drops, Bubbles, Pearls, Waves. Springer, New York, 2004.

[23] P.-G. de Gennes, F. Brochard-Wyart, and D. Quéré. Capillarity and wetting phenomena: Drops, bubbles, pearls, waves. In Capillarity and Wetting Phenomena. Springer, 2004.

[24] J. Doolittle and G. Vali. Heterogeneous freezing nucleation in electric fields. Journal of the Atmospheric Sciences, 32(2):375-379, 1975.

[25] N. E. Dorsey. The freezing of supercooled water. Transactions of the American Philosophical Society, 38(3):247-328, 1948.

[26] A. J. Durant and R. A. Shaw. Evaporation freezing by contact nucleation inside-out. Geophysical Research Letters, 32(20), 2005. 
[27] D. Ehre, E. Lavert, M. Lahav, and I. Lubomirsky. Water freezes differently on positively and negatively charged surfaces of pyroelectric materials. Science, 327(5966):672-675, 2010.

[28] B. Ervens and G. Feingold. Sensitivities of immersion freezing: Reconciling classical nucleation theory and deterministic expressions. Geophysical Research Letters, 40(12):3320-3324, 2013.

[29] J. Fan, S. Ghan, M. Ovchinnikov, X. Liu, P. J. Rasch, and A. Korolev. Representation of arctic mixed-phase clouds and the wegener-bergeron-findeisen process in climate models: Perspectives from a cloud-resolving study. Journal of Geophysical Research: Atmospheres, 116(D1), 2011.

[30] J. Fan, M. Ovtchinnikov, J. M. Comstock, S. A. McFarlane, and A. Khain. Ice formation in arctic mixed-phase clouds: Insights from a 3-d cloud-resolving model with size-resolved aerosol and cloud microphysics. Journal of Geophysical Research: Atmospheres, 114(D4), 2009.

[31] G. Feingold, W. Cotton, B. Stevens, and A. Frisch. The relationship between drop in-cloud residence time and drizzle production in numerically simulated stratocumulus clouds. Journal of the atmospheric sciences, 53(8):1108-1122, 1996.

[32] A. Fridlind, A. Ackerman, G. McFarquhar, G. Zhang, M. Poellot, P. DeMott, A. Prenni, and A. Heymsfield. Ice properties of single-layer stratocumulus 
during the mixed-phase arctic cloud experiment: 2. model results. Journal of Geophysical Research: Atmospheres, 112(D24), 2007.

[33] A. M. Fridlind, B. Van Diedenhoven, A. S. Ackerman, A. Avramov, A. Mrowiec, H. Morrison, P. Zuidema, and M. D. Shupe. A fire-ace/sheba case study of mixed-phase arctic boundary layer clouds: Entrainment rate limitations on rapid primary ice nucleation processes. Journal of the Atmospheric Sciences, 69(1):365-389, 2012.

[34] N. Fukuta. A study of the mechanism of contact ice nucleation. Journal of the Atmospheric Sciences, 32(8):1597-1603, 1975.

[35] B. Garetz, J. Aber, N. Goddard, R. Young, and A. Myerson. Nonphotochemical, polarization-dependent, laser-induced nucleation in supersaturated aqueous urea solutions. Physical review letters, 77(16):3475, 1996.

[36] N. R. Gokhale and J. Goold Jr. Droplet freezing by surface nucleation. Journal of Applied Meteorology, 7(5):870-874, 1968.

[37] N. R. Gokhale and O. Lewinter. Microcinematographic studies of contact nucleation. Journal of Applied Meteorology, 10(3):469-473, 1971.

[38] N. R. Gokhale and J. D. Spengler. Freezing of freely suspended, supercooled water drops by contact nucleation. Journal of Applied Meteorology, 11(1):157160, 1972. 
[39] C. Gurganus, J. Charnawskas, A. Kostinski, and R. Shaw. Nucleation at the contact line observed on nanotextured surfaces. Physical review letters, 113(23):235701, 2014.

[40] C. Gurganus, A. B. Kostinski, and R. A. Shaw. Fast imaging of freezing drops: No preference for nucleation at the contact line. The Journal of Physical Chemistry Letters, 2(12):1449-1454, 2011.

[41] C. Gurganus, A. B. Kostinski, and R. A. Shaw. High-speed imaging of freezing drops: still no preference for the contact line. The Journal of Physical Chemistry C, 117(12):6195-6200, 2013.

[42] E. Hairer, S. P. Nrsett, and G. Wanner. Solving Ordinary Differential Equations: Nonstiff problems. v. 2: Stiff and differential-algebraic problems. Springer Verlag, 2010.

[43] J. Hallett. Experimental studies of the crystallization of supercooled water. Journal of the Atmospheric Sciences, 21(6):671-682, 1964.

[44] R. Hickling. Nucleation of freezing by cavity collapse and its relation to cavitation damage. Nature, 206:915-917, 1965.

[45] C. Hoose, J. E. Kristjánsson, J.-P. Chen, and A. Hazra. A classical-theorybased parameterization of heterogeneous ice nucleation by mineral dust, soot, and biological particles in a global climate model. Journal of the Atmospheric Sciences, 67(8):2483-2503, 2010. 
[46] C. Hoose and O. Möhler. Heterogeneous ice nucleation on atmospheric aerosols: a review of results from laboratory experiments. Atmospheric Chemistry and Physics, 12(20):9817, 2012.

[47] T. Hozumi, A. Saito, S. Okawa, and K. Watanabe. Effects of electrode materials on freezing of supercooled water in electric freeze control. International journal of refrigeration, 26(5):537-542, 2003.

[48] J. Huang and L. S. Bartell. Kinetics of homogeneous nucleation in the freezing of large water clusters. The Journal of Physical Chemistry, 99(12):3924-3931, 1995.

[49] J. Hunt and K. Jackson. Nucleation of solid in an undercooled liquid by cavitation. Journal of Applied Physics, 37(1):254-257, 1966.

[50] J. Hunt and K. Jackson. Nucleation of the solid phase by cavitation in an undercooled liquid which expands on freezing. Nature, 211(5053):1080-1081, 1966.

[51] K. A. Jackson. Kinetic Processes: Crystal Growth, Diffusion, and Phase Transformations in Materials. John Wiley \& Sons, 2006.

[52] R. C. Jackson, G. M. McFarquhar, A. V. Korolev, M. E. Earle, P. S. Liu, R. P. Lawson, S. Brooks, M. Wolde, A. Laskin, and M. Freer. The dependence of ice 
microphysics on aerosol concentration in arctic mixed-phase stratus clouds during isdac and m-pace. Journal of Geophysical Research: Atmospheres, 117(D15), 2012.

[53] H. Kalesse, G. de Boer, A. Solomon, M. Oue, M. Ahlgrimm, D. Zhang, M. D. Shupe, E. Luke, and A. Protat. Understanding rapid changes in phase partitioning between cloud liquid and ice in stratiform mixed-phase clouds: An arctic case study. Monthly Weather Review, 144(12):4805-4826, 2016.

[54] A. Khain, A. Pokrovsky, M. Pinsky, A. Seifert, and V. Phillips. Simulation of effects of atmospheric aerosols on deep turbulent convective clouds using a spectral microphysics mixed-phase cumulus cloud model. part i: Model description and possible applications. Journal of the Atmospheric Sciences, 61(24):2963$2982,2004$.

[55] M. F. Khairoutdinov and D. A. Randall. Cloud resolving modeling of the arm summer 1997 iop: Model formulation, results, uncertainties, and sensitivities. Journal of the Atmospheric Sciences, 60(4):607-625, 2003.

[56] A. Kiselev, F. Bachmann, P. Pedevilla, S. J. Cox, A. Michaelides, D. Gerthsen, and T. Leisner. Active sites in heterogeneous ice nucleationthe example of k-rich feldspars. Science, page aai8034, 2016.

[57] R. G. Knollenberg. A laboratory study of the local cooling resulting from the 
dissolution of soluble ice nuclei having endothermic heats of solution. Journal of the Atmospheric Sciences, 26(1):115-124, 1969.

[58] R. G. Knollenberg. The local cooling ice nucleation model. Journal of the Atmospheric Sciences, 26(1):125-129, 1969.

[59] T. Koop, B. Luo, A. Tsias, and T. Peter. Water activity as the determinant for homogeneous ice nucleation in aqueous solutions. Nature, 406(6796):611-614, 2000.

[60] A. Korolev and P. R. Field. The effect of dynamics on mixed-phase clouds: Theoretical considerations. Journal of the Atmospheric Sciences, 65(1):66-86, 2008.

[61] A. Kostinski and W. Cantrell. Entropic aspects of supercooled droplet freezing. Journal of the Atmospheric Sciences, 65(9):2961-2971, 2008.

[62] M. J. Kreder, J. Alvarenga, P. Kim, and J. Aizenberg. Design of anti-icing surfaces: smooth, textured or slippery? Nature Reviews Materials, 1:15003, 2016.

[63] L. Ladino Moreno, O. Stetzer, and U. Lohmann. Contact freezing: a review of experimental studies. Atmospheric Chemistry and Physics, 13(19):9745-9769, 2013. 
[64] D. Lamb and J. Verlinde. Physics and chemistry of clouds. Cambridge University Press, 2011.

[65] V. E. Larson and A. J. Smith. An analytic scaling law for the depositional growth of snow in thin mixed-phase layer clouds. Journal of the Atmospheric Sciences, 66(9):2620-2639, 2009.

[66] I. Leizerson, S. Lipson, and A. Lyushnin. Wetting properties: When larger drops evaporate faster. Nature, 422(6930):395-396, 2003.

[67] B. Li and D.-W. Sun. Novel methods for rapid freezing and thawing of foods-a review. Journal of food engineering, 54(3):175-182, 2002.

[68] K. Li, S. Xu, J. Chen, Q. Zhang, Y. Zhang, D. Cui, X. Zhou, J. Wang, and Y. Song. Viscosity of interfacial water regulates ice nucleation. Applied Physics Letters, 104(10):101605, 2014.

[69] T. Li, D. Donadio, and G. Galli. Ice nucleation at the nanoscale probes no mans land of water. Nature communications, 4:1887, 2013.

[70] T. Li, D. Donadio, L. M. Ghiringhelli, and G. Galli. Surface-induced crystallization in supercooled tetrahedral liquids. Nature materials, 8(9):726-730, 2009.

[71] Z. Li, H. Xue, and F. Yang. A modeling study of ice formation affected by aerosols. Journal of Geophysical Research: Atmospheres, 118(19), 2013. 
[72] G. Lippmann. Relations entre les phénomènes électriques et capillaires. PhD thesis, Gauthier-Villars, 1875.

[73] D. Lubin and A. M. Vogelmann. The influence of mixed-phase clouds on surface shortwave irradiance during the arctic spring. Journal of Geophysical Research: Atmospheres, 116(D1), 2011.

[74] W. Luedtke, J. Gao, and U. Landman. Dielectric nanodroplets: Structure, stability, thermodynamics, shape transitions and electrocrystallization in applied electric fields. The Journal of Physical Chemistry C, 115(42):20343-20358, 2011.

[75] M. t. Maxey and S. Corrsin. Gravitational settling of aerosol particles in randomly oriented cellular flow fields. Journal of the atmospheric sciences, 43(11):1112-1134, 1986.

[76] G. M. McFarquhar, S. Ghan, J. Verlinde, A. Korolev, J. W. Strapp, B. Schmid, J. M. Tomlinson, M. Wolde, S. D. Brooks, D. Cziczo, et al. Indirect and semidirect aerosol campaign: The impact of arctic aerosols on clouds. Bulletin of the American Meteorological Society, 92(2):183-201, 2011.

[77] H. Morrison, J. Curry, and V. Khvorostyanov. A new double-moment microphysics parameterization for application in cloud and climate models. part i: Description. Journal of the Atmospheric Sciences, 62(6):1665-1677, 2005.

[78] H. Morrison, G. De Boer, G. Feingold, J. Harrington, M. D. Shupe, and K. Sulia. 
Resilience of persistent arctic mixed-phase clouds. Nature Geoscience, 5(1):11$17,2012$.

[79] H. Morrison and W. W. Grabowski. Comparison of bulk and bin warm-rain microphysics models using a kinematic framework. Journal of the atmospheric sciences, 64(8):2839-2861, 2007.

[80] H. Morrison, M. D. Shupe, J. O. Pinto, and J. A. Curry. Possible roles of ice nucleation mode and ice nuclei depletion in the extended lifetime of arctic mixed-phase clouds. Geophysical research letters, 32(18), 2005.

[81] F. Mugele and J.-C. Baret. Electrowetting: from basics to applications. Journal of Physics: Condensed Matter, 17(28):R705, 2005.

[82] T. Němec. Estimation of ice-water interfacial energy based on pressuredependent formulation of classical nucleation theory. Chemical Physics Letters, 583:64-68, 2013.

[83] D. Niedermeier, R. Shaw, S. Hartmann, H. Wex, T. Clauss, J. Voigtländer, and F. Stratmann. Heterogeneous ice nucleation: exploring the transition from stochastic to singular freezing behavior. Atmospheric Chemistry and Physics, 11(16):8767-8775, 2011.

[84] J. Niehaus, J. G. Becker, A. Kostinski, and W. Cantrell. Laboratory measurements of contact freezing by dust and bacteria at temperatures of mixed-phase clouds. Journal of the Atmospheric Sciences, 71(10):3659-3667, 2014. 
[85] J. Niehaus and W. Cantrell. Contact freezing of water by salts. The journal of physical chemistry letters, 6(17):3490-3495, 2015.

[86] D. Ning and X. Liu. Controlled ice nucleation in microsized water droplet. Applied physics letters, 81(3):445-447, 2002.

[87] X. Noblin, A. Buguin, and F. Brochard-Wyart. Vibrated sessile drops: Transition between pinned and mobile contact line oscillations. The European Physical Journal E, 14(4):395-404, 2004.

[88] X. Noblin, A. Buguin, and F. Brochard-Wyart. Triplon modes of puddles. Physical review letters, 94(16):166102, 2005.

[89] Y.-J. Noh, C. J. Seaman, T. H. Vonder Haar, and G. Liu. In situ aircraft measurements of the vertical distribution of liquid and ice water content in midlatitude mixed-phase clouds. Journal of Applied Meteorology and Climatology, 52(1):269-279, 2013.

[90] M. Nosonovsky and B. Bhushan. Phase behavior of capillary bridges: towards nanoscale water phase diagram. Physical Chemistry Chemical Physics, 10(16):2137-2144, 2008.

[91] M. Ovchinnikov, A. S. Ackerman, A. Avramov, A. Cheng, J. Fan, A. M. Fridlind, S. Ghan, J. Harrington, C. Hoose, A. Korolev, et al. Intercomparison of large-eddy simulations of arctic mixed-phase clouds: Importance of ice 
size distribution assumptions. Journal of Advances in Modeling Earth Systems, 6(1):223-248, 2014.

[92] M. Ovchinnikov, A. Korolev, and J. Fan. Effects of ice number concentration on dynamics of a shallow mixed-phase stratiform cloud. Journal of Geophysical Research: Atmospheres, 116(D1), 2011.

[93] A. J. Page and R. P. Sear. Heterogeneous nucleation in and out of pores. Physical review letters, 97(6):065701, 2006.

[94] L. Pauling. General chemistry. Courier Corporation, 1988.

[95] A. Petersen, H. Schneider, G. Rau, and B. Glasmacher. A new approach for freezing of aqueous solutions under active control of the nucleation temperature. Cryobiology, 53(2):248-257, 2006.

[96] J. O. Pinto. Autumnal mixed-phase cloudy boundary layers in the arctic. Journal of the atmospheric sciences, 55(11):2016-2038, 1998.

[97] H. Pruppacher. The effect of an external electric field on the supercooling of water drops. Journal of Geophysical Research, 68(15):4463-4474, 1963.

[98] H. Pruppacher. Interpretation of experimentally determined growth rates of ice crystals in supercooled water. The Journal of Chemical Physics, 47(5):18071813, 1967. 
[99] H. Pruppacher. Electrofreezing of supercooled water. pure and applied geophysics, 104(1):623-634, 1973.

[100] H. R. Pruppacher, J. D. Klett, and P. K. Wang. Microphysics of clouds and precipitation. Taylor \& Francis, 1998.

[101] C. Quilliet and B. Berge. Electrowetting: a recent outbreak. Current Opinion in Colloid \& Interface Science, 6(1):34-39, 2001.

[102] E. Rignot and P. Kanagaratnam. Changes in the velocity structure of the greenland ice sheet. Science, 311(5763):986-990, 2006.

[103] L. J. Rothschild and R. L. Mancinelli. Life in extreme environments. Nature, 409(6823):1092-1101, 2001.

[104] M. Roulleau, L. Evans, and N. Fukuta. The electrical nucleation of ice in supercooled clouds. Journal of the Atmospheric Sciences, 28(5):737-740, 1971.

[105] D. Rzesanke, J. Nadolny, D. Duft, R. Müller, A. Kiselev, and T. Leisner. On the role of surface charges for homogeneous freezing of supercooled water microdroplets. Physical Chemistry Chemical Physics, 14(26):9359-9363, 2012.

[106] R. Salt. Effect of electrostatic field on freezing of supercooled water and insects. Science, 133(3451):458-459, 1961.

[107] V. J. Schaefer. The generation of large numbers of ice crystals in an electric field. Journal of Applied Meteorology, 7(3):452-455, 1968. 
[108] M. Schremb, I. V. Roisman, and C. Tropea. Transient effects in ice nucleation of a water drop impacting onto a cold substrate. Physical Review E, 95(2):022805, 2017.

[109] R. P. Sear. Nucleation at contact lines where fluid-fluid interfaces meet solid surfaces. Journal of Physics: Condensed Matter, 19(46):466106, 2007.

[110] R. P. Sear. The non-classical nucleation of crystals: microscopic mechanisms and applications to molecular crystals, ice and calcium carbonate. International Materials Reviews, 57(6):328-356, 2012.

[111] R. A. Shaw, A. J. Durant, and Y. Mi. Heterogeneous surface crystallization observed in undercooled water. The Journal of Physical Chemistry B, 109(20):9865-9868, 2005.

[112] T. Shichiri and Y. Araki. Nucleation mechanism of ice crystals under electrical effect. Journal of crystal growth, 78(3):502-508, 1986.

[113] T. Shichiri and T. Nagata. Effect of electric currents on the nucleation of ice crystals in the melt. Journal of Crystal Growth, 54(2):207-210, 1981.

[114] B. Shipway and A. Hill. Diagnosis of systematic differences between multiple parametrizations of warm rain microphysics using a kinematic framework. Quarterly Journal of the Royal Meteorological Society, 138(669):2196-2211, 2012 . 
[115] M. Shupe, P. Persson, I. Brooks, M. Tjernström, J. Sedlar, T. Mauritsen, S. Sjogren, and C. Leck. Cloud and boundary layer interactions over the arctic sea ice in late summer. Atmospheric Chemistry and Physics, 13:9379-9400, 2013.

[116] M. D. Shupe, P. Kollias, P. O. G. Persson, and G. M. McFarquhar. Vertical motions in arctic mixed-phase stratiform clouds. Journal of the Atmospheric Sciences, 65(4):1304-1322, 2008.

[117] M. D. Shupe, S. Y. Matrosov, and T. Uttal. Arctic mixed-phase cloud properties derived from surface-based sensors at sheba. Journal of the atmospheric sciences, 63(2):697-711, 2006.

[118] A. Solomon, G. Feingold, and M. Shupe. The role of ice nuclei recycling in the maintenance of cloud ice in arctic mixed-phase stratocumulus. Atmospheric Chemistry and Physics, 15(18):10631-10643, 2015.

[119] A. Solomon, M. Shupe, P. Persson, and H. Morrison. Moisture and dynamical interactions maintaining decoupled arctic mixed-phase stratocumulus in the presence of a humidity inversion. Atmospheric Chemistry and Physics, 11(19):10127-10148, 2011.

[120] C. A. Stan, S. K. Tang, K. J. Bishop, and G. M. Whitesides. Externally applied electric fields up to $1.6 \times 105 \mathrm{v} / \mathrm{m}$ do not affect the homogeneous nucleation of ice in supercooled water. The Journal of Physical Chemistry B, 115(5):10891097, 2010. 
[121] W. Sun, Z. Chen, and S.-y. Huang. Effect of an external electric field on liquid water using molecular dynamics simulation with a flexible potential. Journal of Shanghai University (English Edition), 10(3):268-273, 2006.

[122] I. M. Svishchev and P. G. Kusalik. Crystallization of liquid water in a molecular dynamics simulation. Physical review letters, 73(7):975, 1994.

[123] I. M. Svishchev and P. G. Kusalik. Electrofreezing of liquid water: A microscopic perspective. Journal of the American Chemical Society, 118(3):649-654, 1996.

[124] T. Takahashi, T. Endoh, G. Wakahama, and N. Fukuta. Vapor diffusional growth of free-falling snow crystals between-3 and-23 c. Journal of the Meteorological Society of Japan. Ser. II, 69(1):15-30, 1991.

[125] H. Tan, C. Diddens, P. Lv, J. Kuerten, X. Zhang, and D. Lohse. Evaporationtriggered microdroplet nucleation and the four life phases of an evaporating ouzo drop. Proceedings of the National Academy of Sciences, 113(31):86428647, 2016.

[126] A. R. Ubbelohde. Melting and Crystal Structures. Oxford University Press, 1965.

[127] G. Vali, P. DeMott, O. Möhler, and T. Whale. Technical note: A proposal for ice nucleation terminology. Atmospheric Chemistry and Physics, 15(18):1026310270, 2015. 
[128] M. Vallet, B. Berge, and L. Vovelle. Electrowetting of water and aqueous solutions on poly (ethylene terephthalate) insulating films. Polymer, 37(12):2465$2470,1996$.

[129] M. Vallet, M. Vallade, and B. Berge. Limiting phenomena for the spreading of water on polymer films by electrowetting. The European Physical Journal B-Condensed Matter and Complex Systems, 11(4):583-591, 1999.

[130] A. R. Van Eaton, L. G. Mastin, M. Herzog, H. F. Schwaiger, D. J. Schneider, K. L. Wallace, and A. B. Clarke. Hail formation triggers rapid ash aggregation in volcanic plumes. Nature communications, 6, 2015.

[131] H. Vehkamäki. Classical nucleation theory in multicomponent systems. Springer Science \& Business Media, 2006.

[132] J. Verlinde, J. Y. Harrington, V. Yannuzzi, A. Avramov, S. Greenberg, S. Richardson, C. Bahrmann, G. McFarquhar, G. Zhang, N. Johnson, et al. The mixed-phase arctic cloud experiment. Bulletin of the American Meteorological Society, 88(2):205-221, 2007.

[133] J. M. Wallace and P. V. Hobbs. Atmospheric science: an introductory survey, volume 92. Academic press, 2006.

[134] S. Wei, X. Xiaobin, Z. Hong, and X. Chuanxiang. Effects of dipole polarization of water molecules on ice formation under an electrostatic field. Cryobiology, 56(1):93-99, 2008. 
[135] J. C. Weil, P. P. Sullivan, and C.-H. Moeng. The use of large-eddy simulations in lagrangian particle dispersion models. Journal of the atmospheric sciences, 61(23):2877-2887, 2004.

[136] A. Welti, F. Lüönd, Z. Kanji, O. Stetzer, and U. Lohmann. Time dependence of immersion freezing: an experimental study on size selected kaolinite particles. Atmospheric Chemistry and Physics, 12(20):9893-9907, 2012.

[137] C. Westbrook and A. Illingworth. The formation of ice in a long-lived supercooled layer cloud. Quarterly Journal of the Royal Meteorological Society, 139(677):2209-2221, 2013.

[138] C. D. Westbrook and A. J. Heymsfield. Ice crystals growing from vapor in supercooled clouds between- 2.5 and- 22 c: Testing current parameterization methods using laboratory data. Journal of the Atmospheric Sciences, 68(10):2416-2429, 2011.

[139] S. Wildeman, S. Sterl, C. Sun, and D. Lohse. Fast dynamics of water droplets freezing from the outside in. Physical Review Letters, 118(8):084101, 2017.

[140] P. Wilson, K. Osterday, and A. Haymet. The effects of electric field on ice nucleation may be masked by the inherent stochastic nature of nucleation. CryoLetters, 30(2):96-99, 2009.

[141] T. W. Wilson, L. A. Ladino, P. A. Alpert, M. N. Breckels, I. M. Brooks, J. Browse, S. M. Burrows, K. S. Carslaw, J. A. Huffman, C. Judd, et al. 
A marine biogenic source of atmospheric ice-nucleating particles. Nature, 525(7568):234-238, 2015.

[142] P. Winkler, R. McGraw, P. Bauer, C. Rentenberger, and P. Wagner. Direct determination of three-phase contact line properties on nearly molecular scale. Scientific reports, 6, 2016.

[143] B. Wyslouzil and J. Seinfeld. Nonisothermal homogeneous nucleation. The Journal of chemical physics, 97(4):2661-2670, 1992.

[144] J. Yan, S. Overduin, and G. Patey. Understanding electrofreezing in water simulations. The Journal of chemical physics, 141(7):074501, 2014.

[145] J. Yan and G. Patey. Heterogeneous ice nucleation induced by electric fields. The Journal of Physical Chemistry Letters, 2(20):2555-2559, 2011.

[146] J. Yan and G. Patey. Molecular dynamics simulations of ice nucleation by electric fields. The Journal of Physical Chemistry A, 116(26):7057-7064, 2012.

[147] J. Yan and G. Patey. Ice nucleation by electric surface fields of varying range and geometry. The Journal of chemical physics, 139(14):144501, 2013.

[148] F. Yang, M. Ovchinnikov, and R. A. Shaw. Minimalist model of ice microphysics in mixed-phase stratiform clouds. Geophysical Research Letters, 40(14):37563760, 2013. 
[149] F. Yang, M. Ovchinnikov, and R. A. Shaw. Microphysical consequences of the spatial distribution of ice nucleation in mixed-phase stratiform clouds. Geophysical Research Letters, 41(14):5280-5287, 2014.

[150] F. Yang, M. Ovchinnikov, and R. A. Shaw. Long-lifetime ice particles in mixedphase stratiform clouds: Quasi-steady and recycled growth. Journal of Geophysical Research: Atmospheres, 120(22), 2015.

[151] F. Yang, R. Shaw, and H. Xue. Conditions for super-adiabatic droplet growth after entrainment mixing. Atmospheric Chemistry and Physics, 16(14):94219433, 2016.

[152] F. Yang, R. A. Shaw, C. W. Gurganus, S. K. Chong, and Y. K. Yap. Ice nucleation at the contact line triggered by transient electrowetting fields. Applied Physics Letters, 107(26):264101, 2015.

[153] Y. Yang, G.-W. He, and L.-P. Wang. Effects of subgrid-scale modeling on lagrangian statistics in large-eddy simulation. Journal of Turbulence, 9, 2008.

[154] Z. Zhang, D.-W. Sun, Z. Zhu, and L. Cheng. Enhancement of crystallization processes by power ultrasound: Current state-of-the-art and research advances. Comprehensive Reviews in Food Science and Food Safety, 14(4):303-316, 2015.

[155] Y.-P. Zhao and Y. Wang. Fundamentals and applications of electrowetting. Reviews of Adhesion and Adhesives, 1(1):114-174, 2013. 
[156] X. Zhu, Q. Yuan, and Y.-P. Zhao. Phase transitions of a water overlayer on charged graphene: from electromelting to electrofreezing. Nanoscale, 6(10):5432-5437, 2014. 